\title{
Kepler-36: A Pair of Planets with Neighboring Orbits and Dissimilar Densities
}

\author{
Joshua A. Carter ${ }^{*}{ }^{*}$, Eric Agol ${ }^{2}+^{*}$, William J. Chaplin ${ }^{3}$, \\ Sarbani Basu ${ }^{4}$, Timothy R. Bedding ${ }^{5}$, Lars A. Buchhave ${ }^{6}$, \\ Jørgen Christensen-Dalsgaard ${ }^{7}$, Katherine M. Deck ${ }^{8}$, Yvonne Elsworth ${ }^{3}$, \\ Daniel C. Fabrycky ${ }^{9}$, Eric B. Ford ${ }^{10}$, Jonathan J. Fortney ${ }^{11}$, \\ Steven J. Hale ${ }^{3}$, Rasmus Handberg ${ }^{7}$, Saskia Hekker ${ }^{12}$, \\ Matthew J. Holman ${ }^{13}$, Daniel Huber ${ }^{14}$, Christopher Karoff ${ }^{7}$, \\ Steven D. Kawaler ${ }^{15}$, Hans Kjeldsen ${ }^{7}$, Jack J. Lissauer ${ }^{14}$, \\ Eric D. Lopez ${ }^{11}$, Mikkel N. Lund ${ }^{7}$, Mia Lundkvist ${ }^{7}$, \\ Travis S. Metcalfe ${ }^{16}$, Andrea Miglio ${ }^{3}$, Leslie A. Rogers ${ }^{8}$, \\ Dennis Stello ${ }^{5}$, William J. Borucki ${ }^{14}$, Steve Bryson ${ }^{14}$, \\ Jessie L. Christiansen ${ }^{17}$, William D. Cochran ${ }^{18}$, John C. Geary ${ }^{13}$, \\ Ronald L. Gilliland ${ }^{19}$, Michael R. Haas ${ }^{14}$, Jennifer Hall ${ }^{20}$, \\ Andrew W. Howard ${ }^{21}$, Jon M. Jenkins ${ }^{17}$, Todd Klaus ${ }^{20}$, \\ David G. Koch ${ }^{14}$, David W. Latham ${ }^{13}$, Phillip J. MacQueen ${ }^{18}$, \\ Dimitar Sasselov ${ }^{13}$, Jason H. Steffen ${ }^{22}$, Joseph D. Twicken ${ }^{17}$, \\ Joshua N. Winn ${ }^{8}$
}

${ }_{1}$ Hubble Fellow, Harvard-Smithsonian Center for Astrophysics, 60 Garden Street, Cambridge, MA 02138, USA

${ }^{2}$ Department of Astronomy, Box 351580, University of Washington, Seattle, WA 98195, USA

${ }^{3}$ School of Physics and Astronomy, University of Birmingham, Edgbaston, B15 2TT, UK

${ }^{4}$ Department and Astronomy, Yale University, New Haven, CT, 06520, USA

${ }^{5}$ Sydney Institute for Astronomy, School of Physics, University of Sydney, Sydney, Australia

Niels Bohr Institute, Copenhagen University, DK-2100 Copenhagen, Denmark

${ }^{6}$ Centre for Star and Planet Formation, Natural History Museum of Denmark, University of Copenhagen, DK-1350 Copenhagen, Denmark

${ }_{7}$ Stellar Astrophysics Centre (SAC), Department of Physics and Astronomy, Aarhus University, Ny Munkegade 120, DK-8000 Aarhus C, Denmark 
${ }_{8}$ Massachusetts Institute of Technology, Physics Department and Kavli Institute for Astrophysics and Space Research, 77 Massachusetts Avenue, Cambridge, MA 02139, USA

${ }_{9}$ Hubble Fellow, Department of Astronomy and Astrophysics, University of California, Santa Cruz, CA 95064

${ }^{10}$ Department of Astronomy, University of Florida, Gainesville, FL 32611-2055, USA

${ }^{11}$ Department of Astronomy and Astrophysics, University of California, Santa Cruz, CA 95064

${ }_{12}$ Astronomical Institute 'Anton Pannekoek', University of Amsterdam, The Netherlands School of Physics and Astronomy, University of Birmingham, Edgbaston, B15 2TT, UK

${ }^{13}$ Harvard-Smithsonian Center for Astrophysics, 60 Garden Street, Cambridge, MA 02138, USA

${ }^{14}$ NASA Ames Research Center, Moffett Field, CA 94035, USA

${ }^{15}$ Department of Physics and Astronomy, Iowa State University, Ames, IA, 50011, USA

${ }^{16}$ White Dwarf Research Corporation, Boulder, CO, 80301, USA

${ }^{17}$ SETI Institute/NASA Ames Research Center, Moffett Field, CA 94035

${ }^{18} \mathrm{McD}$ Donald Observatory, University of Texas at Austin, Austin, TX, 78712, USA

${ }_{19}$ Center for Exoplanets and Habitable Worlds, The Pennsylvania State University, University Park, PA, 16802, USA

${ }^{20}$ Orbital Science Corporation/NASA Ames Research Center, Moffett Field, CA 94035

${ }^{21}$ Department of Astronomy, University of California, Berkeley, CA, 94720, USA

${ }^{22}$ Fermilab Center for Particle Astrophysics, P.O. Box 500, Batavia IL 60510, USA

+These authors contributed equally to this work.

*To whom correspondence should be addressed. E-mail:

jacarter@cfa.harvard.edu, agol@astro.washington.edu

In the Solar system the planets' compositions vary with orbital distance, with rocky planets in close orbits and lower-density gas giants in wider orbits. The detection of close-in giant planets around other stars was the first clue that this pattern is not universal, and that planets' orbits can change substantially after their formation. Here we report another violation of the orbit-composition pattern: two planets orbiting the same star with orbital distances differing by only $10 \%$, and densities differing by a factor of 8 . One planet is likely a rocky 'super-Earth', whereas the other is more akin to Neptune. These planets are 


\section{twenty times more closely spaced—and have a larger density contrast—than any adjacent pair of planets in the Solar system.}

The detection of the first 'hot Jupiter' around a Sun-like star (1) was surprising to researchers who based their expectations on the properties of the Solar system. Theorists soon developed models of planetary 'migration' to explain how the planets' orbits can shrink (2). This led to a broader recognition that the architecture of planetary systems can change substantially after their formation. The planetary system reported in this paper is another example of an 'extreme' planetary system that will serve as a stimulus to theories of planet migration and orbital rearrangement. The system features two planets in neighboring orbits with substantially different compositions.

This system was discovered from the miniature eclipses or "transits" that cause the host star to appear fainter when the planets pass in front of the star. The target star (Kepler Object of Interest 277; hereafter, Kepler-36; also KIC 11401755, 2MASS 19250004+4913545) is one of approximately 150,000 stars that is subject to nearly continuous photometric surveillance by the Kepler spacecraft (3-5). The Kepler data revealed the transits of two planets. The loss of light during transits by planet $\mathrm{b}$ is only $17 \%$ as large as that from planet $\mathrm{c}$ (Fig 1). Both transit sequences show substantial timing variations (Fig 2). Indeed the variations are large enough that the smaller planet was initially overlooked by the Kepler data reduction pipeline $(6,7)$, which makes the usual assumption of strictly periodic orbits. It was subsequently identified with a search algorithm allowing for variations between successive transits up to a specified fraction of the mean period (8).

The candidates have average orbital periods of $P_{b}=13.84 \mathrm{~d}$ and $P_{c}=16.23 \mathrm{~d}$, which have a ratio close to $6 / 7$. The transit timing variations are approximately linear in time, with abrupt slope changes after planetary conjunctions $\left(\approx 6 \times P_{b} \approx 7 \times P_{c} \approx\right.$ every $\left.97 \mathrm{~d}\right)$. The variations are anti-correlated; when one body is early, the other is late, and vice versa. This is diagnostic of 
gravitational interactions (9-11), leaving no doubt that the two transiting bodies orbit the same star (as opposed to other scenarios in which two independent eclipsing systems are spatially unresolved by the telescope).

Even without detailed modeling it is possible to show that the transiting bodies must be planets, by imposing the requirement of orbital stability. For long-term stability against collisions or ejections, the sum of the orbiting bodies' masses must be less than $10^{-4}$ times the mass of the star (12-14). This criterion leads to an upper bound of 40 Earth masses, hence planetary masses for both bodies.

Precise knowledge of the masses and radii of the planets requires precise knowledge of those same properties for the star. This information is typically gleaned from spectroscopic observations. In this case there is an additional source of information: the Kepler data reveal solar-like oscillations of the host star, due to excitation of sound waves by turbulence in the stellar atmosphere. The frequencies of the oscillations indicate that the star has a density $(25 \pm$ $2) \%$ that of the Sun $(15,16)$. Analysis of high-resolution spectra of this star, subject to this density constraint, yields precise values for the stellar effective temperature and metallicity. The star is slightly hotter and less metal-rich than the Sun. This information combined with additional asteroseismic constraints (16) gives precise measures of the stellar mass and radius (Table 1). Based on these parameters (16), Kepler-36 is a 'subgiant' star, 2-3 billion years older than the Sun.

We used the seismic results as constraints on the parameters of a photometric-dynamical model fitted to the Kepler data (16). This model is based on the premise of a star and two planets interacting via Newtonian gravity with an appropriate loss of light whenever a planet is projected in front of the star (17). Given initial positions and masses, the positions of the three bodies at the observed times were computed numerically, and the loss of light was computed assuming a linear limb-darkening law (18). Only data within half a day of a transit were fitted, 
after correcting for extraneous trends by removing a linear function of time (16).

We adjusted the parameters of the model to fit to the data. The optimal solution agrees well with the data, in particular with the transit-timing variations of both planets (Fig 1 and Fig 2). We calculated the credible intervals for the model parameters (16) with a Differential Evolution Markov Chain Monte Carlo algorithm (19) (Table 1).

The masses and radii have uncertainties less than $8 \%$ and $3 \%$ respectively. This unusually good precision owes to the combination of the constraint on the planetary mass ratio $(0.55 \pm$ $0.01)$ and planets-to-star mass ratio $\left[(3.51 \pm 0.20) \times 10^{-5}\right]$ obtained from the detection of transittiming variations, coupled with the usual geometric transit parameters and the asteroseismic constraints (16).

Knowledge of the planetary masses and radii enables the study of their possible compositions (16). Planet b's dimensions are consistent with a rocky Earth-like composition, with approximately $30 \%$ of its mass in iron. Any volatile constituents - those having relatively low boiling points such as hydrogen, helium or water - must be a small fraction of b's volume. As a limiting case, if the planet is assumed to have a maximally iron-rich core (20), water may not constitute more than $15 \%$ of the total mass, and any $\mathrm{H} / \mathrm{He}$ atmosphere contributes less than $1 \%$ of the total mass. In contrast, planet c must be volatile-rich; it is less dense than water, implying a substantial $\mathrm{H} / \mathrm{He}$ atmosphere. Taking the interior to be free of water, with an Earth-like composition, the $\mathrm{H} / \mathrm{He}$ atmosphere would contain $9 \%$ of the total mass. Even if the planet were half water by mass, the $\mathrm{H} / \mathrm{He}$ atmosphere would still be $>1 \%$ of the total mass (Fig 3 ).

As for the orbits, the photometric-dynamical model shows (16) they are nearly circular (eccentricities $<0.04$ ) and coplanar (mutual inclination $<2.5$ degrees). The evidence for coplanarity comes from the lack of significant variation in the transit durations $(16,21)$. The orbits are also closely spaced: at conjunctions the planets approach one another within 0.013 AU. The angular size of $\mathrm{c}$ as viewed from $\mathrm{b}$ would be 2.5 times larger than the full Moon viewed 
from Earth. We investigated whether these close encounters are consistent with dynamical stability by scrutinizing a random sample of allowed model parameters (16). Direct numerical integration (16) showed that more than $91 \%$ of this sample avoided disruptive encounters over $\sim 0.7$ million years. From this surviving population, 100 parameter sets were drawn randomly and numerically integrated for 140 million years; none experienced disruptive encounters.

Of all the multiplanet systems for which densities of the planets have been measured, Kepler-36 has the smallest fractional separation between any pair of adjacent orbits, and this pair also has one of the largest density contrasts (Fig S20). These factors present an interesting problem. One would naturally suppose that the two planets formed at widely separated locations in the protoplanetary disk, with volatile-poor b inside the 'snow line' at a few AU, and volatile-rich c outside. It will be interesting to see whether the usual 'migration' mechanism that is invoked to alter planetary orbits - tidal interactions with the gaseous protoplanetary disk - could draw together two planets from such different regions of the disk. Or whether the compositions and densities of the planets could have changed with time, due for example to the preferential erosion of the smaller planet's atmosphere by stellar irradiation (16) (Fig S21). Perhaps a combination of these factors will ultimately explain this puzzling pair of planets.

Acknowledgements NASAs Science Mission Directorate provided funding for the Kepler Discovery mission. JAC and DCF acknowledge support by NASA through Hubble Fellowship grants HF-51267.01-A and HF-51272.01-A awarded by the Space Telescope Science Institute, which is operated by the Association of Universities for Research in Astronomy, Inc., for NASA, under contract NAS 5-26555. EA acknowledges NSF Career grant AST-0645416, and thanks the Center for Astrophysics where this work began. WJC, AM and YE acknowledge the financial support of the UK Science and Technology Facilities Council (STFC). Funding for the Stellar Astrophysics Centre (SAC) is provided by The Danish National Research Founda- 
tion. The research is supported by the ASTERISK project (ASTERoseismic Investigations with SONG and Kepler) funded by the European Research Council (Grant agreement no.: 267864). SH acknowledges financial support from the Netherlands Organisation for Scientific Research (NWO). Computational time on Kraken at the National Institute of Computational Sciences was provided through NSF TeraGrid allocation TG-AST090107. J.N.W. was supported by the NASA Kepler Participating Scientist program through grant NNX12AC76G.

Refer to the supplemental material for access information to data utilized in this work. 


\begin{tabular}{lc} 
Star & \\
\hline Mass, $M_{\star}\left(M_{\odot}\right)$ & $1.071 \pm 0.043$ \\
Radius, $R_{\star}\left(R_{\odot}\right)$ & $1.626 \pm 0.019$ \\
Mean Density, $\rho_{\star}\left(\mathrm{g} \mathrm{cm}^{-3}\right)$ & $0.3508 \pm 0.0056$ \\
Stellar Effective Temperature, $T_{\text {eff }}(\mathrm{K})$ & $5911 \pm 66$ \\
Planet $b$ & \\
\hline Time of Transit, $T_{b}(\mathrm{BJD})$ & $2454960.9753_{-0.0058}^{+0.0055}$ \\
Period, $P_{b}($ day $)$ & $13.83989_{-0.00060}^{+0.0002}$ \\
Orbital Semimajor Axis, $a_{b}(\mathrm{AU})$ & $0.1153 \pm 0.0015$ \\
Mass, $M_{b}\left(M_{\oplus}\right)$ & $4.45_{-0.27}^{+0.33}$ \\
Radius, $R_{b}\left(R_{\oplus}\right)$ & $1.486 \pm 0.035$ \\
Mean Density, $\rho_{b}\left(\mathrm{~g} \mathrm{~cm}{ }^{-3}\right)$ & $7.46_{-0.59}^{+0.74}$ \\
Equilibrium Temperature, $T_{\text {eq,b }}(\mathrm{K})$ & $978 \pm 11$ \\
Planet $c$ & \\
\hline Time of Transit, $T_{c}(\mathrm{BJD})$ & $2454955.9132_{-0.0010}^{+0.0011}$ \\
Period, $P_{c}($ day $)$ & $16.23855_{-0.00054}^{+0.00038}$ \\
Orbital Semimajor Axis, $a_{c}(\mathrm{AU})$ & $0.1283 \pm 0.0016$ \\
Mass, $M_{c}\left(M_{\oplus}\right)$ & $8.08_{-0.46}^{+0.60}$ \\
Radius, $R_{c}\left(R_{\oplus}\right)$ & $3.679 \pm 0.054$ \\
Mean Density, $\rho_{c}\left(\mathrm{~g} \mathrm{~cm}{ }^{-3}\right)$ & $0.89_{-0.05}^{+0.07}$ \\
Equilibrium Temperature, $T_{\text {eq,c }}(\mathrm{K})$ & $928 \pm 10$
\end{tabular}

Table 1: Characteristics of the Kepler-36 system. The stellar parameters are based on the analysis of the optical spectrum and the asteroseismic oscillations observed in the Kepler data (16). Because the orbits are not strictly periodic, the periods and times of transit given here refer to instantaneous ('osculating') values evaluated at an arbitrary reference epoch $(2,454,950$ Barycentric Julian Date, BJD). The parameter ranges are based on the medians and $68 \%$ confidence limits of the marginalized posteriors. The equilibrium temperature was calculated assuming a Bond albedo of $A_{B}=0.3$, with $T_{\text {eq }}=T_{\text {eff }} \sqrt{R_{\star} / 2 a}\left(1-A_{B}\right)^{1 / 4}$. 

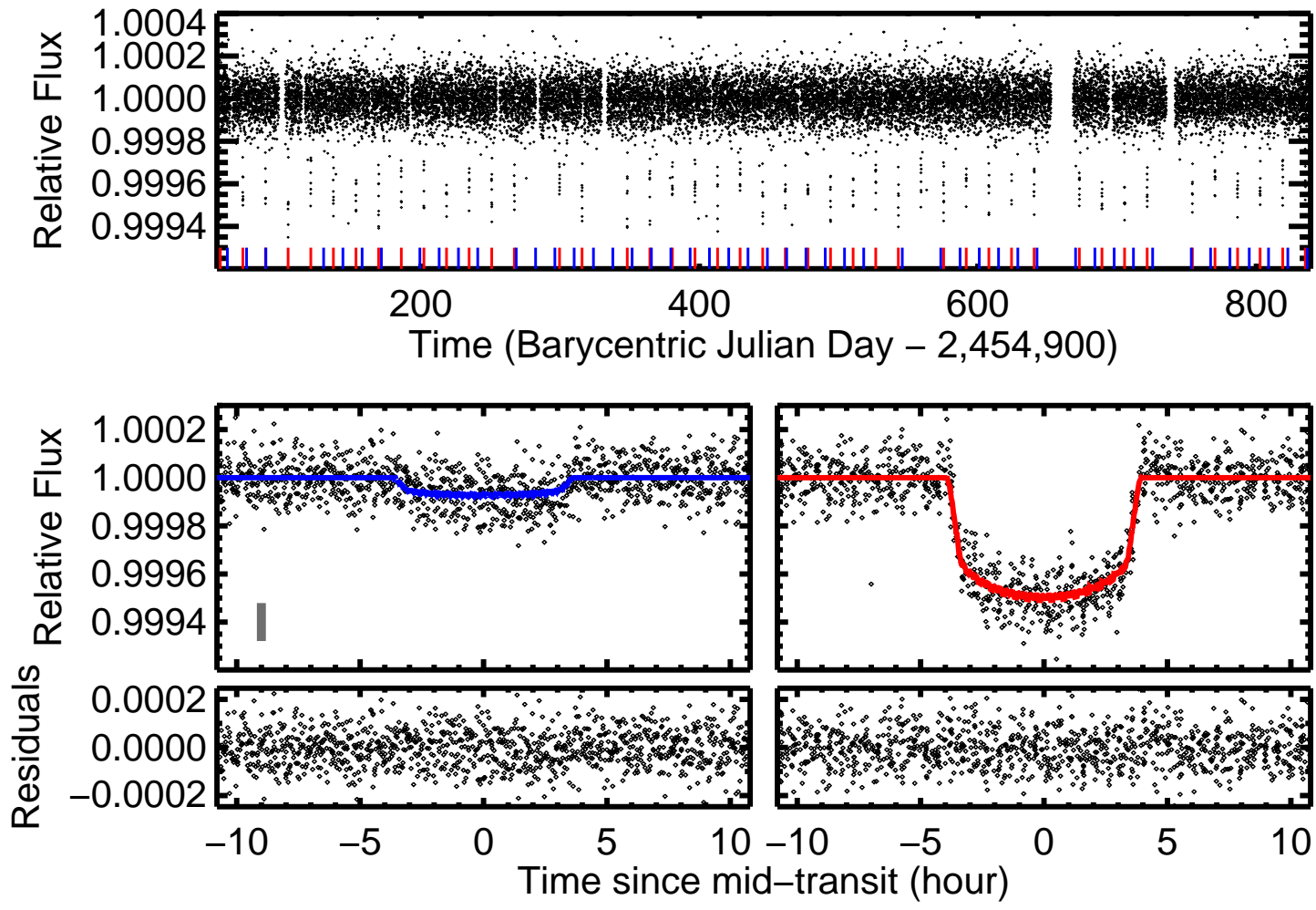

Figure 1: Kepler-36 light curve. Top.-Relative flux over 877 days, after applying a median filter to remove long-term trends caused by astrophysical and instrumental effects. The brief $0.05 \%$ dips represent transits of planet c (also marked with red bars on the time axis). The individual transits of planet $b$ (blue bars) are not easily discerned in the time series. The alternating pattern of transits (blue, red, blue,...) is flipped every $\approx 97$ days as the planets pass each other in their orbits. Bottom.-Composite transit light curves and best-fitting models, obtained by subtracting from each time stamp the nearest mid-transit time (calculated from the best-fitting model), and the data residuals. The gray bar shows the typical scatter of the relative flux. The transit of planet c (right panel, model in red) results in a loss of light about 6 times greater than that due to planet b (left panel, model in blue). 


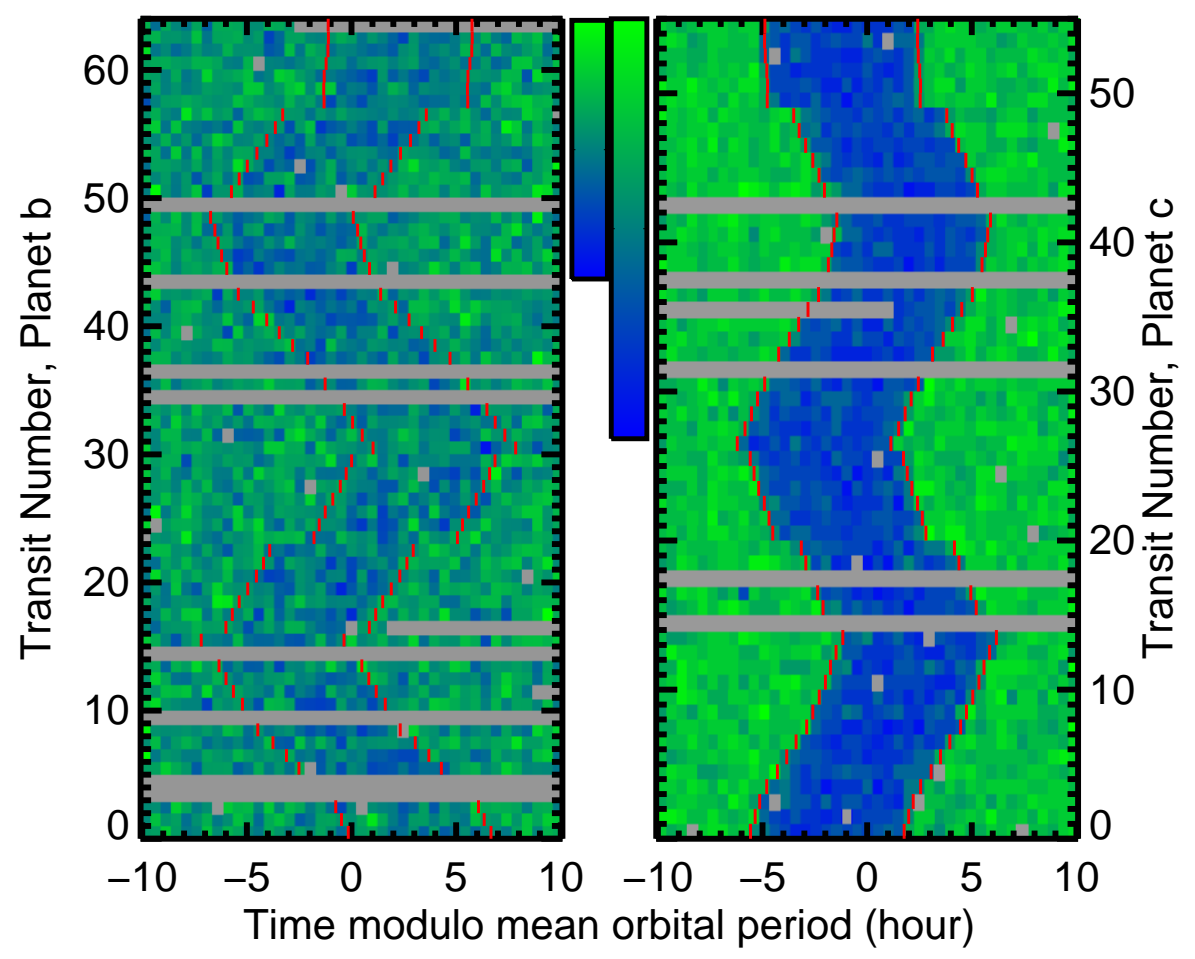

Figure 2: Visualization of transit-timing perturbations. Colored pixels encode relative flux (increasing from blue to green), with light gray pixels representing unavailable data. Each row represents an individual transit light curve, from the earliest observed transit at the bottom to the most recent transit at the top. The horizontal axis is the time modulo the mean orbital period for that planet. Strictly periodic transits would produce a blue vertical band. The curved, riverine bands that are observed are indicative of transit-timing perturbations. The red ticks on each row indicate the start and end time of transit, according to the best-fitting dynamical model. The range of relative fluxes in each panel spans the minimum and maximum data values in the middle panels of Figure 1, 


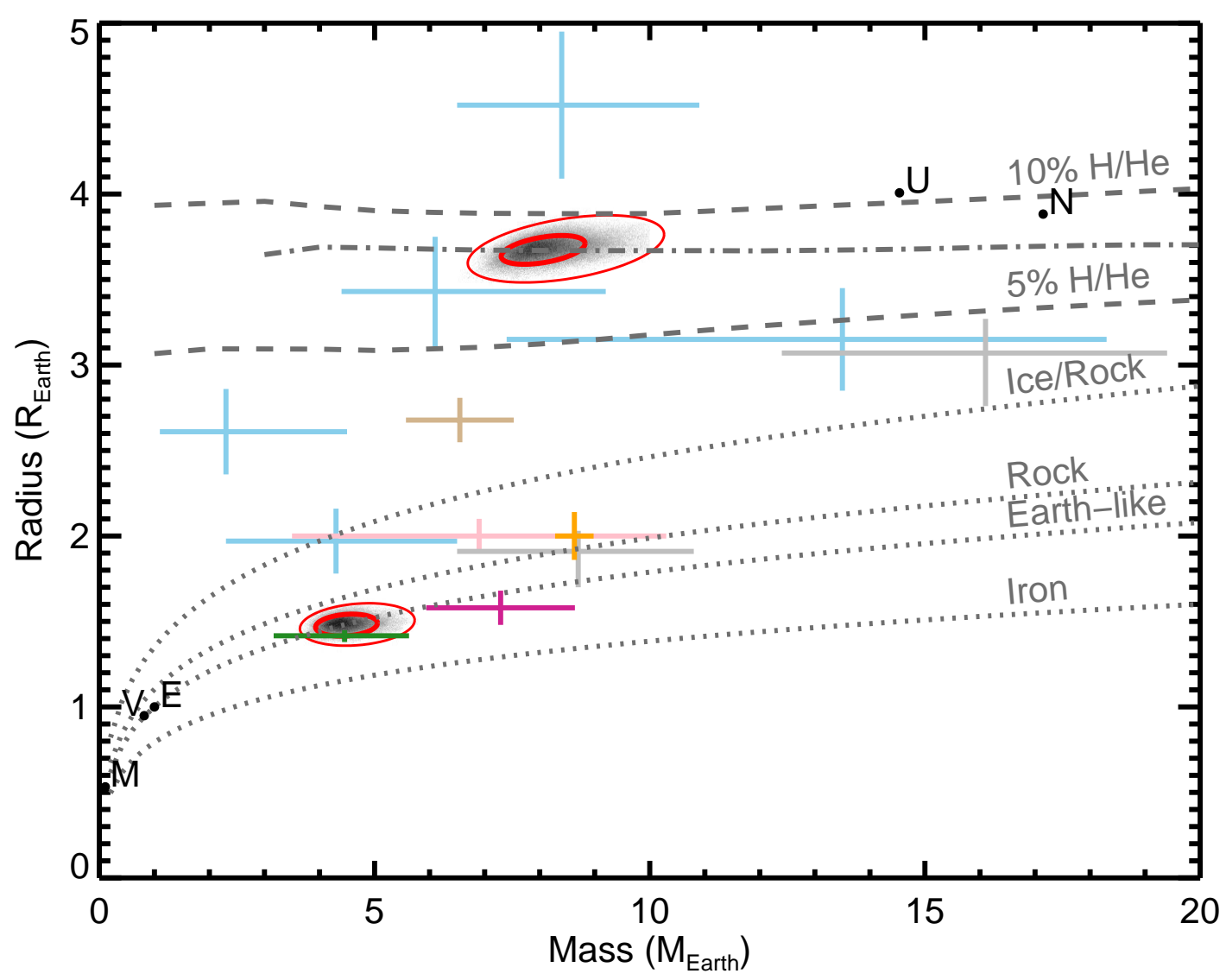

Figure 3: Mass-radius diagram for small planets. Constraints for Kepler-36b and c are shown as two-dimensional joint probability densities and confidence contours (68\% and 95\%). Other exoplanets are shown for comparison: blue - the planets in Kepler-11 (22), pink - Kepler18b (23), gray - Kepler-20 b and c (24), brown - GJ 1214b (25), violet - CoRoT-7b (26), green - Kepler-10b (27), orange - 55 Cnc e (28). Solar System planets are plotted using the first letter of their names (excluding Mercury). The curves represent theoretical models for planets of a given composition. Dotted curves are models of terrestrial bodies [those lacking a significant gas envelope (29)], with "Ice/Rock" - 50\% ice and rock (silicates) by mass, "Rock" - 100\% rock, "Earth-like" - 33\% iron, 67\% rock, "Iron" - 100\% iron. Dashed curves are for planets with Earth-like solid cores surrounded with $\mathrm{H} / \mathrm{He}$ envelopes with $5 \%$ or $10 \%$ of the total mass. The dash-dotted curve is for an Earth-like core and a water layer, in equal parts mass, surrounded by $\mathrm{H} / \mathrm{He}$ envelope with $1.6 \%$ of the total mass. 


\section{Supporting Online Material}

In this supplement, we provide additional details regarding the discovery of Kepler-36 (KOI-277, KIC 11401755, 2MASS 19250004+4913545). This supplement is organized as follows. In $\Varangle 1$, we describe the spectroscopic reconnaissance (\$1.1) and asteroseismology ( $(1.2)$ of Kepler-36 that constrained the stellar properties, summarized in $₫ 1.3 .3$, In $\S 2$, we describe how constraints on the planetary orbits and bulk properties were inferred; we provide both a qualitative discussion $(\$ 2.1)$ and a more detailed description of the photometric-dynamical model $(\$ \underline{2.2})$, model parameters, and the posterior estimation technique. In $₫ 3$, we derive constraints on the composition of the planets based on their bulk densities and thermal histories. We discuss the short-term evolution in $\$ 4$ and long-term stability of Kepler-36 in $₫ 5$. We compare and contrast Kepler-36 with other known planetary systems in $\S$ 6. Finally, in $\S 7$, we describe how to obtain the data used in this analysis and describe attached files containing additional information relevant to this work (including a sample of our MCMC results).

\section{Stellar properties of Kepler-36}

\subsection{Spectroscopy}

\subsubsection{Observations}

Spectroscopic observations to determine the stellar characteristics of Kepler-36 were conducted independently at two observatories. Two spectra were acquired using the Tull Coudé Spectrograph on the $2.7 \mathrm{~m}$ the Harlan J. Smith Telescope at the McDonald Observatory Texas on 29 March 2010 and 8 April 2010. The two spectra were shifted and co-added to provide higher SNR for the spectroscopic analysis. One spectrum was acquired using the HIRES spectrometer on the Keck I 10 m telescope on 11 April 2011.

\subsubsection{Determination of photospheric stellar parameters}

We used the Stellar Parameter Classification (SPC) method (30), to derive the stellar atmosphere parameters from the observed spectra. SPC cross-correlates the observed spectrum against a grid of synthetic spectra drawn from a library calculated by John Laird using Kurucz models (31). The synthetic spectra cover a window of $300 \AA$ centered near the gravity-sensitive $\mathrm{Mg}$ $\mathrm{b}$ features and has a spacing of $250 \mathrm{~K}$ in effective temperature, 0.5 dex in gravity, 0.5 dex in metallicity and a progressive spacing in rotational velocity starting at $1 \mathrm{~km} \mathrm{~s}^{-1}$ up to $20 \mathrm{~km}$ 
$\mathrm{s}^{-1}$. To derive the precise stellar parameters between the grid points, the normalized crosscorrelation peaks were fitted with a three dimensional polynomial as a function of effective temperature, surface gravity and metallicity. This procedure was carried out for different rotational velocities and the final stellar parameters were determined by a weighted mean of the values from the spectral orders covered by the library.

Initial values for the effective temperature and metallicity were determined and used as input in the asteroseismic analysis detailed in Section 1.2 to estimate the surface gravity of the host star. SPC was then re-run, fixing the surface gravity to the value from the asteroseismic analysis $(\log (\mathrm{g})=4.045)$. The final stellar parameters reported here, effective temperature $T_{\text {eff }}=$ $5911 \pm 66 K$, metallicity $[\mathrm{m} / \mathrm{H}]=-0.20 \pm 0.06$ and $v \sin i=4.9 \pm 1.0 \mathrm{~km}^{-1}$, are the mean values derived from the co-added McDonald spectrum. The HIRES spectrum was provided late to this analysis, but its parameters agree with those derived from the McDonald spectrum.

\subsection{Asteroseismic analysis of Kepler-36}

The asteroseismic analysis of Kepler-36 was performed by the Kepler Asteroseismic Science Operations Centre (KASOC) team.

\subsubsection{Estimation of asteroseismic parameters}

The analysis was based upon 15 months of Kepler short-cadence (1-minute sampled) data, collected between 2010 March 2 and 2011 June 26. Fig. \$1 plots the frequency-power spectrum of the light curve (the planetary transit signals having first been removed). It shows a clear pattern of peaks due to solar-like oscillations that are acoustic (pressure, or $\mathrm{p}$ ) modes of high radial order, $n$. The observed power in the oscillations is modulated in frequency by an envelope that has an approximately Gaussian shape. The frequency of maximum oscillation power, $\nu_{\max }$, has been shown to scale to good approximation as $g T_{\text {eff }}^{-1 / 2}(32,33)$, where $g$ is the surface gravity and $T_{\text {eff }}$ is the effective temperature of the star. The most obvious spacings in the spectrum are the large frequency separations, $\Delta \nu$, between consecutive overtones $n$ of the same spherical angular degree, $l$. These large separations scale to very good approximation as $\langle\rho\rangle^{1 / 2}$, $\langle\rho\rangle \propto M / R^{3}$ being the mean density of the star with mass $M$ and surface radius $R$ (34).

We used four independent analysis codes to obtain estimates of the average large separation, $\langle\Delta \nu\rangle$, and $\nu_{\max }$, using automated analysis tools that have been developed, and extensively tested (35-38) for application to Kepler data (15). A final value of each parameter was selected by taking the individual estimate that lay closest to the average over all teams. The uncertainty on the final value was given by adding (in quadrature) the uncertainty on the chosen estimate and the standard deviation over all teams. Excellent agreement was found between the results. The final values for $\langle\Delta \nu\rangle$ and $\nu_{\max }$ were $67.9 \pm 1.2 \mu \mathrm{Hz}$ and $1250 \pm 44 \mu \mathrm{Hz}$, respectively. 


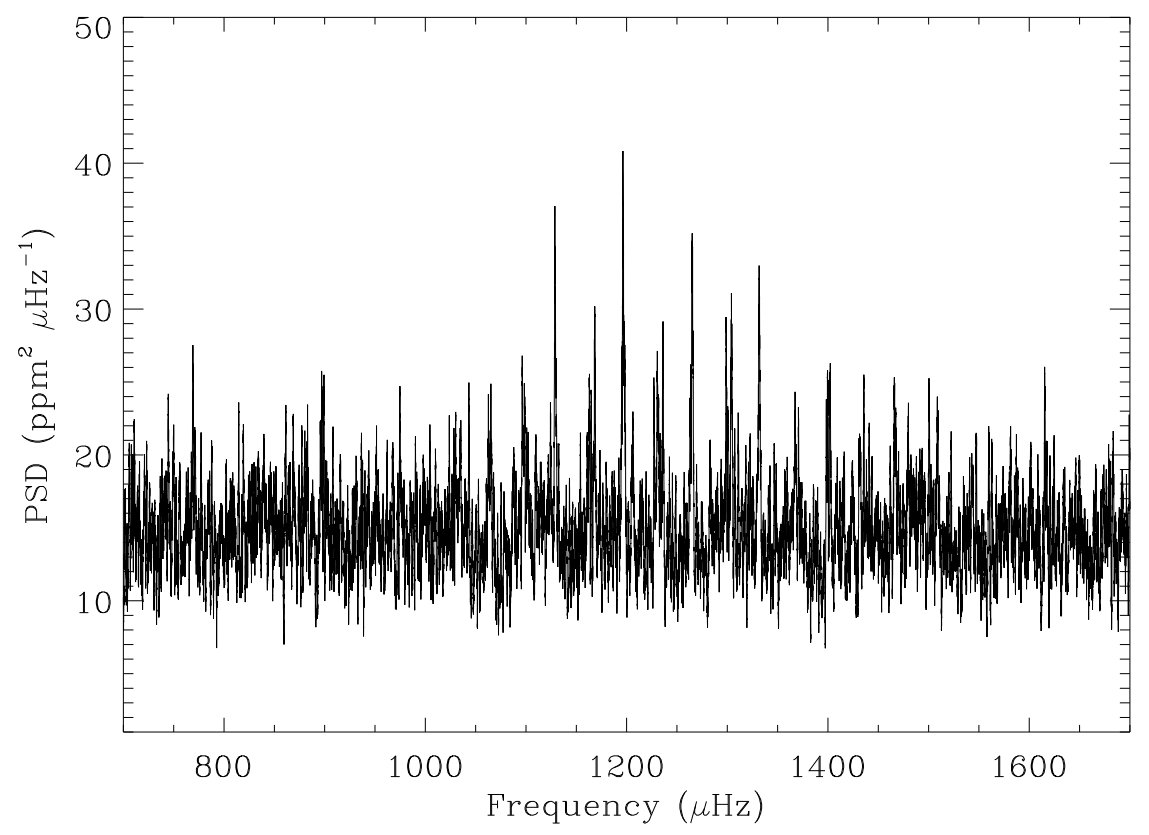

Figure S1: Frequency-power spectrum of Kepler-36, showing a pattern of nearly equally-spaced overtones of solar-like oscillations.

Use of individual frequencies increases the information content provided by the seismic data for making inference on the stellar properties. We therefore also applied "peak bagging" to the frequency-power spectrum to extract estimates of the mode frequencies. Two codes were applied, one using a pseudo-global approach with maximum likelihood estimation (39); and another which performed a global fitting using Markov chain Monte Carlo (MCMC) (40). As per the average seismic parameters, we found excellent agreement in the fitted frequencies between the two approaches. The frequencies, $\nu_{n l}^{\mathrm{obs}}$, and their associated uncertainties, $\sigma_{n l}^{\mathrm{obs}}$, are listed in Table 1.2.1. They were taken from the MCMC analysis, which provides more conservative frequency uncertainties.

\subsection{Estimation of stellar properties}

The analysis was divided into two parts.

\subsubsection{Grid-based modeling with average asteroseismic parameters}

In the first part we used a grid-based approach, in which properties were determined by searching among a grid of stellar evolutionary models to get a best fit for the input parameters, which 
Table S1: Estimated frequencies $\nu_{n l}^{\mathrm{obs}}$ and uncertainties $\sigma_{n l}^{\mathrm{obs}}$ (68\% credible region) of Kepler-36 (in $\mu \mathrm{Hz}$ ).

\begin{tabular}{cccc}
\hline$n$ & $l=0$ & $l=1$ & $l=2$ \\
14 & & $1063.98_{-1.03}^{+0.96}$ & $1095.98_{-5.08}^{+1.54}$ \\
15 & $1099.15_{-1.22}^{+1.59}$ & $1129.06_{-0.28}^{+0.28}$ & $1162.36_{-0.66}^{+0.67}$ \\
16 & $1168.35_{-0.44}^{+0.35}$ & $1196.75_{-0.25}^{+0.25}$ & $1230.63_{-0.95}^{+0.56}$ \\
17 & $1235.86_{-0.56}^{+0.30}$ & $1264.75_{-0.38}^{+0.33}$ & $1298.86_{-0.65}^{+0.59}$ \\
18 & $1304.01_{-0.28}^{+0.29}$ & $1331.62_{-0.43}^{+0.39}$ & \\
19 & & $1400.23_{-0.71}^{+0.93}$ & \\
20 & & $1466.60_{-0.88}^{+1.28}$ & \\
\hline
\end{tabular}


were $\langle\Delta \nu\rangle, \nu_{\max }$, and the spectroscopically estimated $T_{\text {eff }}$ and $[\mathrm{Fe} / \mathrm{H}]$ of the star. Descriptions of the grid-based pipelines used in the analysis may be found in (41-44). An important output of this first part was not only a guideline set of stellar properties - which could be used as the starting points for detailed modeling of the individual frequencies in the second part of the analysis - but also iterated, and improved estimates of $T_{\text {eff }}$ and $[\mathrm{Fe} / \mathrm{H}]$. Initial values of the spectroscopic data were used together with the average seismic properties to estimate $\log g$. The spectroscopic analysis was then repeated with $\log g$ fixed at this value, to yield the revised (and final) values of $T_{\text {eff }}=5911 \pm 66 \mathrm{~K}$ and $[\mathrm{Fe} / \mathrm{H}]=-0.20 \pm 0.06$. Convergence of the inferred properties (to within the estimated uncertainties) was achieved after this one iteration.

\subsubsection{Modeling with individual mode frequencies}

In the second and final part of the analysis, the individual frequencies $\nu_{n l}^{\text {obs }}$ were used as the seismic inputs to a detailed modeling performed by four members of the KASOC team (TM, JCD, AM and SB). The spectroscopic inputs were also used, as per the grid-pipeline analysis. Estimated stellar properties from the first, grid-based part were used either as starting guesses or as a guideline check for initial results. Each analysis sought to minimize a standard $\chi^{2}$ metric. A separate value of $\chi^{2}$ was calculated for the asteroseismic and spectroscopic constraints, and these values are averaged for the final quality metric.

TM used the Asteroseismic Modeling Portal (AMP), a web-based tool tied to TeraGrid computing resources that uses a parallel genetic algorithm (45) to optimize, in an automated manner, the match to observational data $(46,47)$.AMP employs the Aarhus stellar evolution code ASTEC (48) and adiabatic pulsation code ADIPLS (49). Models were made using the OPAL 2005 equation of state and the most recent OPAL opacities supplemented by opacities at low temperature (50), nuclear reaction rates from (51), and helium diffusion and settling following (52). Convection was treated with standard mixing-length theory without overshooting (53).

Each AMP model evaluation involves the computation of a stellar evolution track from the zero-age main sequence (ZAMS) through a mass-dependent number of internal time steps, terminating prior to the beginning of the red giant stage. Exploiting the fact that $\langle\Delta \nu\rangle$ is a monotonically decreasing function of age (46), the asteroseismic age is optimized along each evolutionary track using a binary decision tree. The frequencies of the resulting model are then corrected for surface effects following the prescription of (54). The optimal model is then subjected to a local analysis that uses singular value decomposition (SVD) to quantify the uncertainties of the final model parameters (55).

JCD applied a fitting technique that has been used for the analysis of the Hubble observations of HD 17156 (56), and Kepler observations of the Kepler exoplanet host stars HAT-P7 (35) and Kepler-10 (57). The stellar modeling was carried out with the ASTEC code (48). The calculations used the OPAL equation of state tables (58) and OPAL opacities at tempera- 
tures above $10^{4} \mathrm{~K}(59)$; at lower temperature the opacities (60) were used. Nuclear reactions were calculated using the NACRE parameters (61). Convection was treated using the (53) mixing-length formulation. Frequencies were computed for the models using ADIPLS (49). The prescription in (54) was again applied in an attempt to deal with the surface term.

For each evolutionary sequence in the grid of ASTEC models, the model $\mathcal{M}_{\min }^{\prime}$ whose surface-corrected frequencies provided the best $\chi^{2}$ match to the observations was selected. The best match was obtained from application of homology scaling, i.e., it was assumed that frequencies in the vicinity of $\mathcal{M}_{\text {min }}^{\prime}$ could be obtained as $r \nu_{n l}\left(\mathcal{M}_{\min }^{\prime}\right)$, where $r=\left[R / R\left(\mathcal{M}_{\min }^{\prime}\right)\right]^{-1.5}$, $R$ being the surface radius of the model. A best-fitting model was then determined by minimizing the sum $\sum\left(\nu_{n l}^{\text {obs }}-r \nu_{n l}\left(\mathcal{M}_{\text {min }}^{\prime}\right)\right)^{2} /\left(\sigma_{n l}^{\text {obs }}\right)^{2}$ over all observed modes, as a function of $r$. The resulting value of $r$ defines an estimate of the radius of the best-fitting model along the given sequence. The other properties of this best-fitting model are then determined by linear interpolation in $R$, to the radius of the best-fitting model. Statistical analysis of the ensemble of best-fitting properties from all evolutionary sequences then yielded the final stellar properties, and their uncertainties. A Monte Carlo simulation, involving 100 realizations of the observed frequencies with the addition of normally distributed errors having the inferred standard deviations, showed that the observational errors on the frequencies make a modest contribution to the errors in the inferred stellar properties.

AM used stellar models computed with the CLES code (62), from the pre-main sequence up to the sub-giant branch. For each evolutionary track adiabatic oscillations were computed for about 120 main-sequence and sub-giant models with the LOSC code (63) Grids of models both without and with diffusion and settling of helium and heavy elements (see (62) were considered. The opacity tables are those of OPAL96 (59) complemented at $T<10^{4} \mathrm{~K}$ with the opacities of (60). The metal mixture used in the opacity tables was the solar one, as per (64). The nuclear energy generation routines are based on the cross-sections by NACRE (61), with the OPAL equation of state (65). Convection transport was treated with the classical mixing length approximation of (53).

The analysis was performed in a similar manner to JCD, including treatment of the surface term using the prescription (54), and interpolation in $R$ close to the $\chi^{2}$ minimum to find the best-fitting properties.

SB made use of the Yale stellar evolution code, YREC (66) to model the star. The input physics included the OPAL equation of state tables of (65), and OPAL high-temperature opacities (59) supplemented with low -temperature opacities from (60). All nuclear reaction rates were from (67), except for the rate of the ${ }^{14} \mathrm{~N}(p, \gamma){ }^{15} \mathrm{O}$ reaction, which was fixed at the value of (68). Models were constructed for two values of core overshoot, 0 and $0.2 H_{p}$. Two families of models were constructed, one that included the diffusion and settling of helium and heavy elements as per the the formulation of (69), and one that did not include any diffusion and 
settling.

YREC was used in an iterative mode. In this mode the final $T_{\text {eff }}$ and radius for a model of a given mass and metallicity was specified, and for a given mixing length parameter $\alpha$ the code iterated over the initial helium abundance $Y_{0}$ until a model with the specified $T_{\text {eff }}$ and radius was found. Note that this is similar to the construction of standard solar models, though in that case the iteration is performed over both the mixing length parameter and $Y_{0}$ (since the solar age is a known constraint). Since the age of Kepler-36 is not known independently, the iteration over $Y_{0}$ was done for many different values of the mixing length parameter. All solutions for which the initial helium abundance was less than the primordial helium abundance, $Y_{\mathrm{p}}$ were rejected. $Y_{\mathrm{p}}$ was assumed to be 0.245 .

The surface term correction was handled in a manner that is slightly different from the other analyses. The first step was the construction of a standard solar model with exactly the same physics as that used to model Kepler-36. This yielded the set $\nu_{n l \odot}$ of solar model frequencies. These were then used to estimate a set of "surface term" frequency offsets, $\delta \nu_{n l \odot}$, for the Sun by computing differences between the solar model frequencies and the solar low-degree frequencies observed by the Birmingham Solar Oscillations Network (BiSON), as in (70).

Then, for each stellar model $\mathcal{M}^{\prime}$ under consideration, $\nu_{n l \odot}$ and $\delta \nu_{n l \odot}$ were scaled to the mass and radius of $\mathcal{M}^{\prime}$ using the homology scaling $r=\left\langle\Delta \nu\left(\mathcal{M}^{\prime}\right)\right\rangle /\left\langle\Delta \nu_{n l, \odot}\right\rangle$. The resulting $r \nu_{n l \odot}-r \delta \nu_{n l \odot}$ relation was then used to correct the stellar model for the surface term. Using a

least squares minimization a factor $\beta$ was selected so as to minimize $\sum\left(\nu_{n l}^{\text {obs }}-\nu_{n l}^{\text {corr }}\right)^{2} /\left(\sigma_{n l}^{\text {obs }}\right)^{2}$ over all observed modes, where $\nu_{n l}^{\text {corr }}=\nu_{n l}^{\mathcal{M}^{\prime}}+\beta r \delta \nu_{n l \odot}$, with $r \delta \nu_{n l \odot}$ evaluated at $r \nu_{n l \odot}=\nu_{n l}^{\text {obs }}$.

\subsubsection{Results on stellar properties}

Kepler-36 turned out to be a non-trivial star to model. The star is quite evolved, and a fraction of the models that can potentially provide a reasonable match to the observations show mixed $l=2$ modes in the frequency range where estimated frequencies are provided, thereby giving the potential to complicate the analysis $(71,72)$. Since none of the observed frequencies showed evidence of strong mixing, model frequencies identified as those with the highest inertia, which did not satisfy asymptotic behaviour, e.g. $(73,74)$, were eliminated from the analysis. Some modifications were also made to the (54) surface-term prescription. This prescription was developed for application to radial modes: However, since for Kepler-36 the observed radialmode data in Table 1.2.1 does not extend to the highest frequencies, JCD adapted the method so it could be applied to the non-radial modes. The correction was based on an estimate of the large separation $\langle\Delta \nu\rangle$ and an average frequency obtained by fitting to all the observed modes.

In spite of the above potential complications, differences in the treatment of the surface term, and differences in the physics inputs, there was excellent agreement in the properties returned by 
all four analyses. For each of $M, R, \log g$, mean density $\langle\rho\rangle$, and age $\tau$, the standard deviation of the four estimates was significantly lower than the median formal uncertainty of the property.

Our final properties are those of the modeler whose estimates lay closest to the median (and mean) over all modelers. For the uncertainties we took the chosen modeler's uncertainty for each property and added (in quadrature) the standard deviation of the property from the different modeling results. We find $M=1.071 \pm 0.043 \mathrm{M}_{\odot}, R=1.626 \pm 0.019 \mathrm{R}_{\odot},\langle\rho\rangle=0.3508 \pm$ $0.0056 \mathrm{~g} \mathrm{~cm}^{-3}, \log g=4.045 \pm 0.009 \mathrm{dex}$ and $\tau=6.8 \pm 1.0$ Gyr. (We add that the initial, grid-modeling-based estimates agreed to within uncertainties) Kepler-36 has a luminosity 2.9 times that of the Sun and an approximate distance of $470 \mathrm{pc}$.

\subsection{Photometric determination of the stellar rotation period?}

The full Kepler photometry demonstrates a significant modulation with a period of $17.20 \pm$ 0.2 days (Figure $\mathbf{S 2}$ ). This period corresponds to the rotational period of Kepler-36 if this modulation is due to the "transit" of photospheric surface features. In fact, this measured period agrees well with the maximum rotational period; we find $P_{\text {rot,max }}=16.8 \pm 4.3$ days assuming the results from above of $v \sin i_{\star}=4.9 \pm 1.0 \mathrm{~km} / \mathrm{s}$ and $R_{\star}=1.626 \pm 0.019$. A 17.20 day rotational period is typical (75) for subgiant stars with the same $B-V$ color as Kepler-36 (estimated to be $B-V \approx 0.55$ ). We note that this period is close to the orbital period of Planet c (at 16.28 days). However, it is extremely unlikely that the star has been tidally synchronized with c given its mass and distance. The correspondence is likely coincidental.

\section{Constraints on Planetary Orbits, Masses and Radii}

In this section we describe in detail how we derive the properties of the planets and the orbits from the photometric data.

\subsection{Qualitative Considerations}

Mass ratios between the planets and the star may be quickly estimated based on the qualitative behavior of the variations of the times of transit relative to a constant period model [i.e., the functional form of the transit timing variations (TTVs); see Figure [S3, Tables $[\mathbf{S 2}$ and $[\mathbf{S 3}]$, as described as follows.

The planetary mass ratio can be estimated assuming conservation of energy. Since the planets interact gravitationally, if they do not experience a close approach, they each maintain nearly Keplerian orbits. This suggests that

$$
E_{\text {orbital }} \approx E_{b}+E_{c} \approx-\frac{G M_{\star} M_{b}}{2 a_{b}}-\frac{G M_{\star} M_{c}}{2 a_{c}} \approx \text { const. }
$$




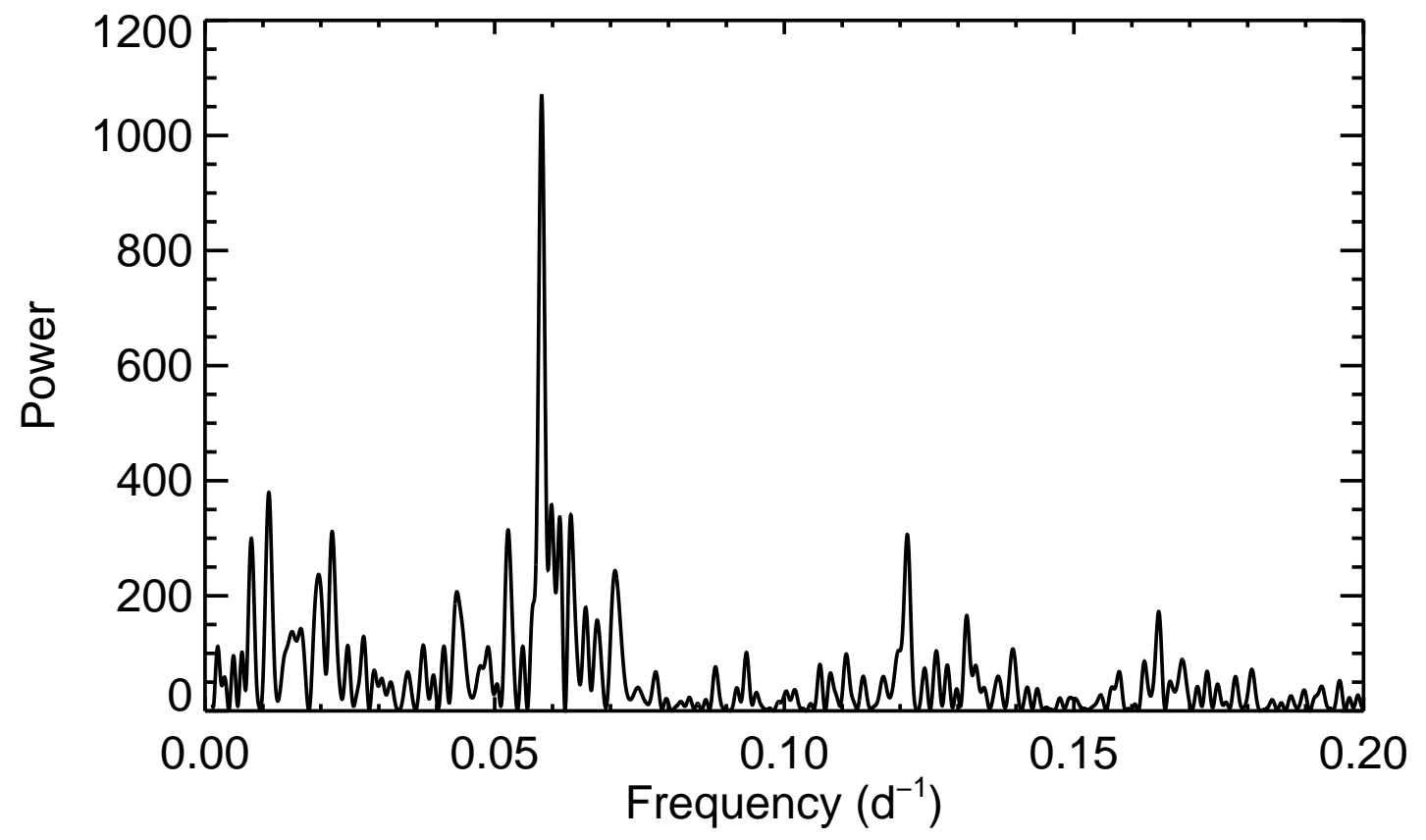

Figure S2: Periodogram of the Kepler photometry at low frequencies demonstrating a strong peak at $0.058 \mathrm{~d}^{-1}$ or 17.20 days. This may represent the rotational period of Kepler-36; it agrees with the maximum rotational period determined from $v \sin i$ and the radius of Kepler-36. 


\begin{tabular}{lcc}
\hline Epochs & $\begin{array}{c}\text { Ephemeris, } T_{0} \\
(\text { BJD-2,454,900) }\end{array}$ & Period, $P$ (days) \\
\hline $0,1,2$, & $60.9771 \pm 0.0760$ & $13.8073 \pm 0.0725$ \\
$5,6,7,8$, & $130.1465 \pm 0.0576$ & $13.8174 \pm 0.0196$ \\
$10,11,12,13,15$, & $199.2772 \pm 0.0546$ & $13.8310 \pm 0.0145$ \\
$16,17,18,19,20,21,22$, & $282.3275 \pm 0.0169$ & $13.8648 \pm 0.0044$ \\
23, 24, 25, 26, 27, 28, 29, & $379.4499 \pm 0.0368$ & $13.8673 \pm 0.0090$ \\
30, 31, 32, 33, 35, & $476.5412 \pm 0.0107$ & $13.8187 \pm 0.0033$ \\
37, 38, 39, 40, 41, 42, & $573.3318 \pm 0.0037$ & $13.8244 \pm 0.0010$ \\
$44,45,46,47,48$, & $670.1201 \pm 0.0046$ & $13.8423 \pm 0.0016$ \\
50, 51, 52, 53, 54, 55, 56, & $753.2429 \pm 0.0044$ & $13.8645 \pm 0.0010$ \\
57, 58, 59, 60, 61, 62, 63, & $850.3652 \pm 0.0044$ & $13.8512 \pm 0.0012$ \\
\hline
\end{tabular}

Table S2: Linear ephemerides for Planet b, measured independently from the Kepler photometry. The ephemeris listed is that for the first epoch in the set under "Epochs."

This equation neglects the interaction energy of the two planets as well as the the planet masses relative to the star in computing the orbital periods. Since $a_{b} / a_{c} \approx\left(P_{b} / P_{c}\right)^{2 / 3}$,

$$
\frac{M_{b}}{P_{b}^{2 / 3}} \approx-\frac{M_{c}}{P_{c}^{2 / 3}}+\text { const. }
$$

Perturbing this equation gives

$$
\frac{M_{c}}{M_{b}} \approx-\frac{\delta P_{b}}{\delta P_{c}}\left(\frac{P_{c}^{5 / 3}}{P_{b}^{5 / 3}}\right) .
$$

This equation assumes that the energy exchange between the planets is small compared to their orbital energies (i.e. that there are no close encounters). Investigating Figure $2, \delta P_{b} \approx 8.5 \mathrm{hr}$ and $\delta P_{c} \approx 5.5 \mathrm{hr}$, while $P_{c} / P_{b} \approx 7 / 6 \approx 1.17$, so $M_{c} / M_{b} \approx 2$.

The ratio of the sum of the planet masses to the star can be derived from the total amplitude of the TTVs and the period of circulation (76). The best-fit periods are $P_{b}=13.85$ days and $P_{c}=16.23$ days and have a proximity to resonance of $\epsilon=1-(j+1) P_{b} /\left(j P_{c}\right) \approx 0.005$ where $j=6$ in this case.

This distance from resonance causes the conjunctions to drift in inertial space over $N_{\text {conj }} \approx$ $j^{-2} \epsilon^{-1} \approx 5$ conjunctions. As can be seen in Figure 2 , it takes $\approx 5$ conjunctions (e.g. from 150 to 600 days when the TTV traces cross one another in the same direction) to complete a TTV cycle.

The semi-amplitude of the sum of the TTVs is $\delta t \approx 7$ hours. Using equation 29 from (76), this gives a total mass ratio of

$$
\mu \equiv \frac{M_{b}+M_{c}}{M_{\star}}=\sqrt{\frac{\delta t \epsilon^{3}}{P_{b}}} \approx 5 \times 10^{-5} .
$$


This equation assumes that period variations dominate the transit timing variations (which can be seen in the riverplot as the jumps at conjunctions are smaller than the TTV due to changes in period between conjunctions). It also assumes plane-parallel planets of much smaller mass than the star, and neglects some factors that are of order unity.

Resonance is expected for $\epsilon<j^{1 / 3} \mu^{2 / 3}=0.0024$, which is not satisfied for the $6: 7$ commensurability. The best-fit solution's (described in $\S 2.2 .5$ ) resonant argument does not librate.

Table S3: Planet c mid-transit times, measured independently from the Kepler photometry.

\begin{tabular}{lc} 
Epoch & $\begin{array}{c}\text { Mid-transit time } \\
\text { (BJD - 2,454,900) }\end{array}$ \\
\hline 0 & $55.9135 \pm 0.0042$ \\
1 & $72.1543 \pm 0.0041$ \\
2 & $88.3951 \pm 0.0071$ \\
3 & $104.6409 \pm 0.0034$ \\
4 & $120.8930 \pm 0.0039$ \\
5 & $137.1427 \pm 0.0030$ \\
6 & $153.3924 \pm 0.0028$ \\
7 & $169.6447 \pm 0.0036$ \\
8 & $185.8876 \pm 0.0040$ \\
9 & $202.1310 \pm 0.0043$ \\
10 & $218.3740 \pm 0.0041$ \\
11 & $234.6149 \pm 0.0059$ \\
12 & $250.8567 \pm 0.0058$ \\
13 & $267.1010 \pm 0.0043$ \\
15 & $299.5228 \pm 0.0051$ \\
16 & $315.7430 \pm 0.0035$ \\
18 & $348.1813 \pm 0.0032$ \\
19 & $364.4032 \pm 0.0027$ \\
20 & $380.5782 \pm 0.0039$ \\
21 & $396.8023 \pm 0.0041$ \\
22 & $413.0243 \pm 0.0065$ \\
23 & $429.2448 \pm 0.0046$ \\
24 & $445.4671 \pm 0.0045$ \\
25 & $461.6911 \pm 0.0067$ \\
26 & $477.8979 \pm 0.0056$ \\
27 & $494.1446 \pm 0.0048$ \\
28 & $510.3889 \pm 0.0053$ \\
29 & $526.6322 \pm 0.0044$
\end{tabular}


Table S3: continued...

\begin{tabular}{lc} 
Epoch & $\begin{array}{c}\text { Mid-transit time } \\
\text { (BJD - 2,454,900) }\end{array}$ \\
\hline 30 & $542.8778 \pm 0.0040$ \\
32 & $575.3683 \pm 0.0039$ \\
33 & $591.6204 \pm 0.0030$ \\
34 & $607.8700 \pm 0.0056$ \\
35 & $624.1196 \pm 0.0033$ \\
36 & $640.3718 \pm 0.0035$ \\
38 & $672.8535 \pm 0.0038$ \\
39 & $689.0915 \pm 0.0029$ \\
40 & $705.3274 \pm 0.0041$ \\
41 & $721.5641 \pm 0.0037$ \\
43 & $754.0018 \pm 0.0029$ \\
44 & $770.2221 \pm 0.0028$ \\
45 & $786.4410 \pm 0.0039$ \\
46 & $802.6580 \pm 0.0031$ \\
47 & $818.8763 \pm 0.0033$ \\
48 & $835.0969 \pm 0.0038$ \\
49 & $851.2757 \pm 0.0048$ \\
50 & $867.5075 \pm 0.0055$ \\
51 & $883.7375 \pm 0.0050$ \\
52 & $899.9658 \pm 0.0048$ \\
53 & $916.1958 \pm 0.0051$ \\
54 & $932.4275 \pm 0.0041$ \\
\hline &
\end{tabular}

\subsection{Photometric-Dynamical Model}

We modeled the Kepler light curve of Kepler-36 using a dynamical model to predict the motions of the planets, and a transit model to predict the light curve. We performed an initial fit to the measured times of transit (given in Tables $\mathbf{S} 2$ and $\mathbf{S 3}$ ), using the dynamical model alone, to seed a subsequent fit with the full photometric-dynamical model.

\subsubsection{Description of the model}

The "photometric-dynamical model" refers to the model that was used to fit the Kepler photometry. This model is equivalent to that described in the analyses of KOI-126 (17), Kepler-16 (77), Kepler-34 and Kepler-35 (78).

The underlying model was a gravitational three-body integration. This integration utilized a 


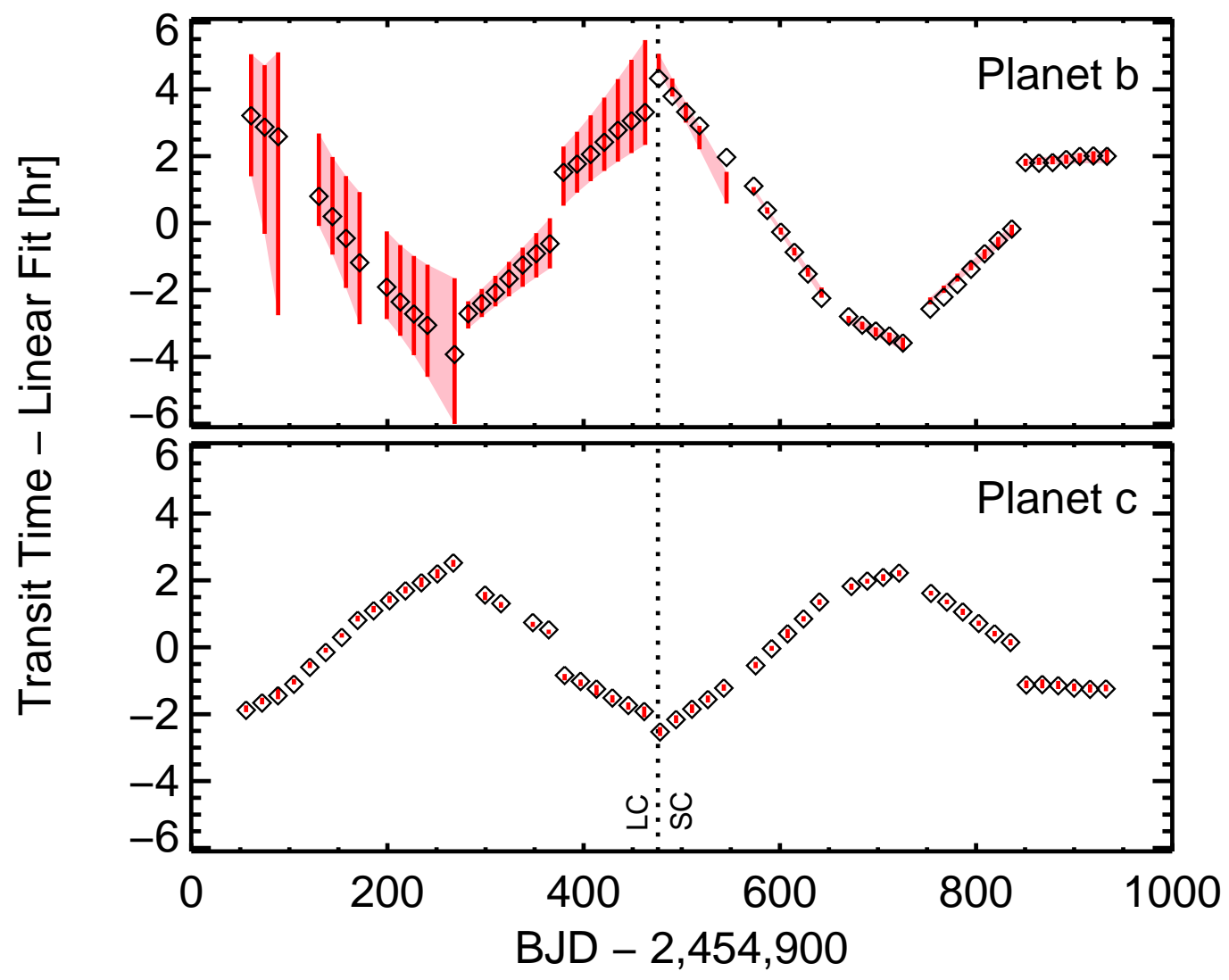

Figure S3: Transit times of the two planets as predicted from a fit assuming a linear ephemeris between each conjunction for Planet $\mathrm{b}$ and individual epoch transit times for Planet $\mathrm{c}$. The error bars represent the uncertainty on each transit time. Due to the smaller transit depth for Planet $\mathrm{b}$, the uncertainties are much larger than for planet $\mathrm{c}$. The dotted vertical line indicates the time at which short cadence data began to be collected for Kepler-36; the short cadence data yield much more precise times of transit. The open diamonds are the best-fit times according to the photodynamical model $(\S 2.2)$. The typical uncertainty in the predicted times are 13 minutes and 3 minute for the transits of planet $b$ and planet $c$, respectively. 
hierarchical (or Jacobian) coordinate system. In this system, $\mathbf{r}_{\mathbf{b}}$ is the position of Planet $\mathbf{b}$ relative to the star, and $r_{c}$ is the position of Planet $c$ relative to the center of mass of Planet $b$ and the star. The computations are performed in a Cartesian system, although it is convenient to express $\mathbf{r}_{\mathrm{b}}$ and $\mathbf{r}_{\mathrm{c}}$ and their time derivatives in terms of osculating Keplerian orbital elements: instantaneous period, eccentricity, argument of pericenter, inclination, longitude of the ascending node, and time of transit: $P_{b, c}, e_{b, c}, i_{b, c}, \omega_{b, c}, \Omega_{b, c}, T_{b, c}$, respectively. We note that these parameters do not necessarily reflect observables in the light curve; the unique three-body effects make these parameters functions of time. The "time of transit," in particular, does not exactly correspond to an measured transit time; exact correspondence would only be seen if the orbit proceeded in a Keplerian fashion from the reference epoch.

The accelerations of the three bodies are determined from Newton's equations of motion, which depend on $\mathbf{r}_{\mathrm{b}}, \mathbf{r}_{\mathrm{c}}$ and the masses $(79,80)$. For the purpose of reporting the masses and radii in Solar units, we assumed $G M_{\text {Sun }}=2.959122 \times 10^{-4} \mathrm{AU}^{3}$ day $^{-2}$ and $R_{\text {Sun }}=0.00465116$ AU. We used a Bulirsch-Stoer algorithm (81) to integrate the coupled first-order differential equations for $\dot{\mathbf{r}}_{b, c}$ and $\mathbf{r}_{b, c}$.

We did not determine the spatial coordinates of all three bodies at each observed time. Instead, to speed computation, we recorded only the sky-plane projected separation between star and planet and the sky-plane projected speed of planet relative to star at the calculated time of transit (for a given epoch). The times of transit were solved for numerically as being those times when the projected separation between the star and planet was minimized. The result of these calculations was a collection of transit times $t_{i_{k}}^{k}$, impact parameters $b_{i_{k}}^{k}$ and speeds $v_{i_{k}}^{k}$ for each planet $k \in\{b, c\}$ and for epochs $i_{k} \in N_{k}$ where $N_{k}$ is the set of observed epoch numbers for planet $k$. The motion of the planet relative to the star is approximately linear in the sky-plane such that the projected separation as a function of time is, to good approximation,

$$
Z_{i_{k}}^{k}(t)=\sqrt{\left[v_{i_{k}}^{k}\left(t-t_{i_{k}}^{k}\right)\right]^{2}+\left(b_{i_{k}}^{k}\right)^{2}}
$$

for times near (a few transit durations) of the calculated mid-transit time.

The approximate photometric model for the relative stellar flux, $f(t)$, is then defined as

$$
f(t)=1-\sum_{k} \sum_{i_{k} \in N_{k}} \begin{cases}\lambda\left(Z_{i_{k}}^{k}(t), R^{k}, u\right)[1+c(t)]^{-1} & -0.5 \leq t-t_{i_{k}}^{k} \leq 0.5 \\ 0 & \text { otherwise }\end{cases}
$$

where $\lambda(z, r, u)$ is the overlap integral between a limb darkened star of radius $R_{\star}$ (such that the radial brightness profile is $I\left(\rho / R_{\star}\right) / I(0)=1-u\left[1-\sqrt{1-\left(\rho / R_{\star}\right)^{2}}\right]$ with linear limbdarkening parameter $u$ ) whose center is separated by a distance $z$ from a dark, opaque sphere of radius $r$. The function $c(t)$ is a piecewise constant function giving the extra flux, relative to the stellar flux, that is included in the photometric aperture and is assumed to be constant in each 
Kepler quarter. $\lambda(z, r, u)$ may be computed semi-analytically with available codes (18). This photometric model, assuming constant transit velocity, is faster to compute than calculating the positions at each photometric cadence and results in a negligible change in the quality of the model fit to the data compared to exact integration. This model does not include the "anomalous" brightening events that occur when the Planet c occults Planet b during a transit (82). No such events are predicted within the current observations.

The continuous model $f(t)$ is integrated over a 29.4 minutes interval centered on each long cadence sample. The continuous model is compared "as is" to the short cadence observations.

\subsubsection{Local detrending of Kepler data}

The Kepler light curve ("SAP_FLUX" from the standard fits product) for Kepler-36, spanning ten Quarters (877 days), is reduced to only those data within 0.5 day of any transit of either planet. Only long cadence data (29.4 minute cadence) are available for the first 406 days of the available Kepler data. Short cadence data (1 minute cadence) are available for the remaining 456 days. Short cadence data are used when available. Transits are missing only when Kepler data are unavailable. Data are missing as a result of observation breaks during quarterly data transfers or spacecraft safe modes.

Each continuous segment of data (being those data observed within 0.5 day) has a local linear correction divided into it. The parameters of this linear correction are found through an iterative process, as described as follows. In the first step, we masked the higher signal-tonoise transits of planet $\mathrm{c}$ and then performed a robust linear least-squares fit to each continuous segment. The data, having divided out this correction, were then fit with the photo-dynamical model with a nonlinear fitter (Levenberg-Marquardt). The best-fit model was then used to identify cadences in transit which were then masked and the fit and division was repeated. This process was repeated until the corrections converged to a sufficient tolerance.

We also experimented with performing this correction inline with the posterior estimation routine (described in $\$ 2.2 .6$ but found negligible differences compared to leaving these fixed according to the converged linear correction found from the process described above.

\subsubsection{Specification of parameters}

The reference epoch was chosen to be $t_{0}=2,454,950$ (BJD), near a particular transit (a somewhat arbitrary choice).

The model has 20 adjustable parameters. Two parameters are related to stellar constraints from asteroseismology and are subject to the priors discussed in $\$ 1.3 .3$, the stellar density times the gravitational constant, $G \rho_{\star}$, and the stellar radius, $R_{\star}$. Two parameters are the mass ratios $q_{+} \equiv\left(M_{b}+M_{c}\right) / M_{\star}$ and $q_{p} \equiv M_{b} / M_{c}$. Four parameters encode the eccentricities and 
arguments of pericenter in a nonlinear way that reduces the complexity of the posterior topology (resulting in effectively linear correlations in these parameters):

$$
\begin{aligned}
h_{-} & \equiv 0.895 \times e_{b} \cos \omega_{b}-e_{c} \cos \omega_{c} \\
h_{+} & \equiv e_{b} \cos \omega_{b}+e_{c} \cos \omega_{c} \\
k_{-} & \equiv e_{b} \sin \omega_{b}-e_{c} \sin \omega_{c} \\
k_{+} & \equiv e_{b} \sin \omega_{b}+e_{c} \sin \omega_{c}
\end{aligned}
$$

In hindsight, we should have used a different linear combination of parameters, namely $a_{b} \mathbf{e}_{\mathbf{b}} \pm a_{c} \mathbf{e}_{\mathbf{c}}$, where $\mathbf{e}_{\mathbf{i}}=e_{i}\left(\cos \omega_{i}, \sin \omega_{i}\right)$. This is due to the fact that the transit timing constrains most strongly the distance at closest approach and orbital phase of the planets at closest approach. This is a function of the epicyclic amplitude and phase which is proportional to $a_{i} \mathbf{e}_{\mathbf{i}}$ for each planet. The differences are well constrained since these determine the closest approach for the planets, while the sums are poorly constrained. The only gain in re-executing our analysis with this parameterization would be more rapid convergence to the target distribution (via the method described in $\$ 2.2 .6$. Having already reached sufficient convergence, we did not pursue this.

The remaining osculating parameters, 7 in total, are the periods $P_{b}, P_{c}$, the orbital inclinations $i_{b}, i_{c}$, the times of transit $T_{b}, T_{c}$ and the difference between the nodal longitudes $\Delta \Omega \equiv \Omega_{c}-\Omega_{b}$. The absolute nodal angle relative to North cannot be determined.

Two more parameters are the relative radii of the planets: $r_{b} \equiv R_{b} / R_{\star}$ and $r_{c} \equiv R_{c} / R_{\star}$. One parameter, $u$, parameterizes the linear limb darkening law for the star (described above). The final two parameters describe the width of the probability distribution for the photometric noise of the long cadence and short cadence observations, assumed to be stationary, white and Gaussian-distributed ( $\sigma_{\mathrm{LC}}$ and $\sigma_{\mathrm{SC}}-$ see the next section).

Additionally, we may specify 9 more parameters describing the function $c(t)$, introduced above, describing the relative extra flux summed in the aperture. The nine parameters specify the constant extra flux in each Kepler quarter. There are 10 quarters in total, but the absolute level of this contamination cannot be determined from the photometry alone, but, the levels relative to a single quarter may be specified. We chose this reference quarter to be quarter 7 having observed in preliminary fits that this quarter's contamination fraction was near the median of the sample of 10 quarters. The addition of these degrees of freedom did not significantly change the distribution of the 20 primary parameters.

We note that a discrete degeneracy exists between the relative nodal angle, $\Delta \Omega$, and inclinations, $i_{b}$ and $i_{c}$. The light curve model is invariant under the transformation $\left(\Delta \Omega, i_{b}, i_{c}\right) \rightarrow$ $\left(-\Delta \Omega, \pi-i_{b}, \pi-i_{c}\right)$. Consequently, we expect the marginalized posterior distribution of $\Delta \Omega$ to be symmetric about $\Delta \Omega=0$, and the distributions of $i_{b}, i_{c}$ to be symmetric about $\pi / 2$. 


\subsubsection{Priors and Likelihood}

We assumed uniform priors in 16 of the 20 primary parameters described in the previous section excluding $h_{+,-}$and $k_{+,-}$. For these latter four parameters, we enforced uniform priors in eccentricities and arguments of pericenter. For these priors, the probability density obeys

$$
p\left(h_{+,-}, k_{+,-}\right) d h_{+,-} k_{+,-} \propto p\left(e_{b, c}, \omega_{b, c}\right) \times \frac{1}{e_{b} e_{c}} d_{e_{b}, e_{c}} d_{\omega_{b}, \omega_{c}} \propto \frac{1}{e_{b} e_{c}} d_{e_{b}, e_{c}} d_{\omega_{b}, \omega_{c}}
$$

The likelihood $\mathcal{L}$ of a given set of parameters was taken to be the product of likelihoods based on the photometric data, assumed-Gaussian asteroseismology priors, and the weighting to enforce priors (described above):

$$
\begin{aligned}
\mathcal{L} \propto & \sigma_{\mathrm{LC}}^{-N_{\mathrm{LC}}} \exp \left[-\sum_{i}^{N_{\mathrm{LC}}} \frac{\left(\Delta F_{i}^{L C}\right)^{2}}{2 \sigma_{\mathrm{LC}}^{2}}\right] \times \sigma_{\mathrm{SC}}^{-N_{\mathrm{SC}}} \exp \left[-\sum_{i}^{N_{\mathrm{SC}}} \frac{\left(\Delta F_{i}^{S C}\right)^{2}}{2 \sigma_{\mathrm{SC}}^{2}}\right] \\
& \times \exp \left[\frac{1}{2}\left(\frac{\Delta G \rho_{\star}}{\sigma_{G \rho_{\star}}}\right)^{2}\right] \times \exp \left[\frac{1}{2}\left(\frac{\Delta R_{\star}}{\sigma_{R_{\star}}}\right)^{2}\right] \times \frac{1}{e_{b} e_{c}}
\end{aligned}
$$

where $\Delta F_{i}^{\mathrm{LC}, \mathrm{SC}}$ is the $i$ th photometric data residual (either long cadence or short cadence), $\Delta G \rho_{\star} / \sigma_{G \rho_{\star}}$ and $\Delta R_{\star} / \sigma_{R_{\star}}$ are the deviates between the asteroseismic constraints in density and radius, and $\sigma_{\mathrm{LC}, \mathrm{SC}}$ are the width parameters describing the photometric noise for either long or short cadence data. These noise parameters have values that are within a fraction of a percent of the root-mean-square normalized flux as calculated using the Kepler light curve, excluding the data near any transit.

\subsubsection{Best-fit model}

We determined a best-fit model by maximizing the likelihood $\mathcal{L}$ as defined above. The maximum likelihood solution was found by determining the highest likelihood in a large draw from the posterior as calculated with a Markov Chain Monte Carlo simulation as described in $\$ 2.2 .6$,

Figure \$4 shows the data folded on the best-fit period for both planets, as in Figure 2, as well as the analogous map for the best-fit photometric-dynamical model. The transit direction has been scaled by the ratio $7: 6$ such that the transits of the planets adjacent to one another correspond to nearly coincident times. The gravitational kicks imparted by the planets on one another are apparent after each conjunction. The purple dots mark the best-fit time of transit at each epoch; the best-fit times are listed in Tables S4 and S5. The best-fit model has $\chi^{2}=99640.7$ for 99718 degrees of freedom. If we ignore the transits of $b$ in the model (or equivalently we let the radius of $\mathrm{b}$ be zero) we find $\Delta \chi^{2}=498$; this corresponds to a detection of $\mathrm{b}$ at $22 \sigma$. 
Table S4: Planet b mid-transit times from the best-fitting photometric-dynamical model.

\begin{tabular}{lc} 
Epoch & $\begin{array}{c}\text { Mid-transit time } \\
\text { (BJD - 2,454,900) }\end{array}$ \\
\hline 0 & 60.9794 \\
1 & 74.8155 \\
2 & 88.6539 \\
5 & 130.1295 \\
6 & 143.9542 \\
7 & 157.7770 \\
8 & 171.5968 \\
10 & 199.2660 \\
11 & 213.0977 \\
12 & 226.9327 \\
13 & 240.7685 \\
15 & 268.4324 \\
16 & 282.3319 \\
17 & 296.1944 \\
18 & 310.0579 \\
19 & 323.9246 \\
20 & 337.7915 \\
21 & 351.6553 \\
22 & 365.5175 \\
23 & 379.4552 \\
24 & 393.3153 \\
25 & 407.1771 \\
26 & 421.0420 \\
27 & 434.9066 \\
28 & 448.7681 \\
29 & 462.6286 \\
30 & 476.5201 \\
31 & 490.3483 \\
32 & 504.1788 \\
33 & 518.0120 \\
35 & 545.6733 \\
37 & 573.3377 \\
38 & 587.1575 \\
39 & 600.9805 \\
40 & 614.8053 \\
41 & 628.6283 \\
& \\
\hline 3 &
\end{tabular}


Table S4: continued...

\begin{tabular}{cc} 
Epoch & $\begin{array}{c}\text { Mid-transit time } \\
\text { (BJD - 2,454,900) }\end{array}$ \\
\hline 42 & 642.4481 \\
44 & 670.1250 \\
45 & 683.9643 \\
46 & 697.8070 \\
47 & 711.6506 \\
48 & 725.4916 \\
50 & 753.2334 \\
51 & 767.0986 \\
52 & 780.9644 \\
53 & 794.8334 \\
54 & 808.7028 \\
55 & 822.5694 \\
56 & 836.4339 \\
57 & 850.3660 \\
58 & 864.2151 \\
59 & 878.0656 \\
60 & 891.9193 \\
61 & 905.7728 \\
62 & 919.6233 \\
63 & 933.4727 \\
\hline
\end{tabular}

Table S5: Planet c mid-transit times from the best-fitting photometric-dynamical model.

\begin{tabular}{lc} 
Epoch & $\begin{array}{c}\text { Mid-transit time } \\
\text { (BJD - 2,454,900) }\end{array}$ \\
\hline 0 & 55.9117 \\
1 & 72.1520 \\
2 & 88.3923 \\
3 & 104.6379 \\
4 & 120.8902 \\
5 & 137.1401 \\
6 & 153.3900 \\
7 & 169.6425 \\
8 & 185.8856 \\
9 & 202.1295 \\
10 & 218.3731
\end{tabular}


Table S5: continued...

\begin{tabular}{cc} 
Epoch & $\begin{array}{c}\text { Mid-transit time } \\
\text { (BJD - } 2,454,900)\end{array}$ \\
\hline 11 & 234.6144 \\
12 & 250.8568 \\
13 & 267.1016 \\
15 & 299.5242 \\
16 & 315.7447 \\
18 & 348.1836 \\
19 & 364.4059 \\
20 & 380.5804 \\
21 & 396.8042 \\
22 & 413.0258 \\
23 & 429.2460 \\
24 & 445.4680 \\
25 & 461.6917 \\
26 & 477.8975 \\
27 & 494.1442 \\
28 & 510.3884 \\
29 & 526.6316 \\
30 & 542.8771 \\
32 & 575.3677 \\
33 & 591.6200 \\
34 & 607.8698 \\
35 & 624.1196 \\
36 & 640.3720 \\
38 & 672.8539 \\
39 & 689.0919 \\
40 & 705.3277 \\
41 & 721.5644 \\
43 & 754.0017 \\
44 & 770.2221 \\
45 & 786.4409 \\
46 & 802.6579 \\
47 & 818.8762 \\
48 & 835.0968 \\
49 & 851.2751 \\
50 & 867.5069 \\
51 & 883.7367 \\
52 & 899.9649 \\
& \\
\hline 3 &
\end{tabular}


Table S5: continued...

Epoch Mid-transit time

\begin{tabular}{lc} 
& (BJD - 2,454,900) \\
\hline 53 & 916.1948 \\
54 & 932.4265 \\
\hline
\end{tabular}

Figure 5 5 shows a plot of the normalized data versus the best-fit photometric-dynamical model. The correlation is very nearly linear showing that the model is an excellent fit to the normalized data.

Finally, we have created a set of panels of every transit with the best-fit model in Figures [56 and S7, Figure S6 shows the long cadence data while Figure S7 shows the short cadence data. We also have created a movie (Movie S1), attached with this supplement, which shows the transits of planet $\mathrm{c}$ as a function of time.

The parameters of the best-fit model are listed in Table $\mathbf{S 6}$ and derived parameters from that best-fit solution are listed in Table S7, In $\$ 4.1$, we describe the short-term evolution of the best-fit solution.

\subsubsection{Parameter estimation methodology}

We explored the parameter space and estimated the posterior parameter distribution with a Differential Evolution Markov Chain Monte Carlo (DE-MCMC) algorithm (19). In this algorithm, a large population of independent Markov chains are calculated in parallel. As in a traditional MCMC, links are added to each chain in the population by proposing parameter jumps, and then accepting or denying a jump from the current state according to the Metropolis-Hastings criterion, using the likelihood function given in $\$ 2.2 .4$. What is different from a traditional MCMC is the manner in which jump sizes and directions are chosen for the proposals. A population member's individual parameter jump vector at step $i+1$ is calculated by selecting two randomly chosen population members (not including itself), and then forming the difference vector between their parameter states at step $i$ and scaling by a factor $\Gamma$. This is the Differential Evolution component of the algorithm. The factor $\Gamma$ is adjusted such that the fraction of accepted jumps, averaged over the whole population, is approximately $25 \%$.

We generated a population of 60 chains and evolved through approximately 500,000 generations. The initial parameter states of the 60 chains were randomly selected from an overdispersed region in parameter space bounding the final posterior distribution. The first $10 \%$ of the links in each individual Markov chain were clipped, and the resulting chains were concatenated to form a single Markov chain, after having confirmed that each chain had converged according to the standard criteria including the Gelman-Rubin convergence statistics and the 

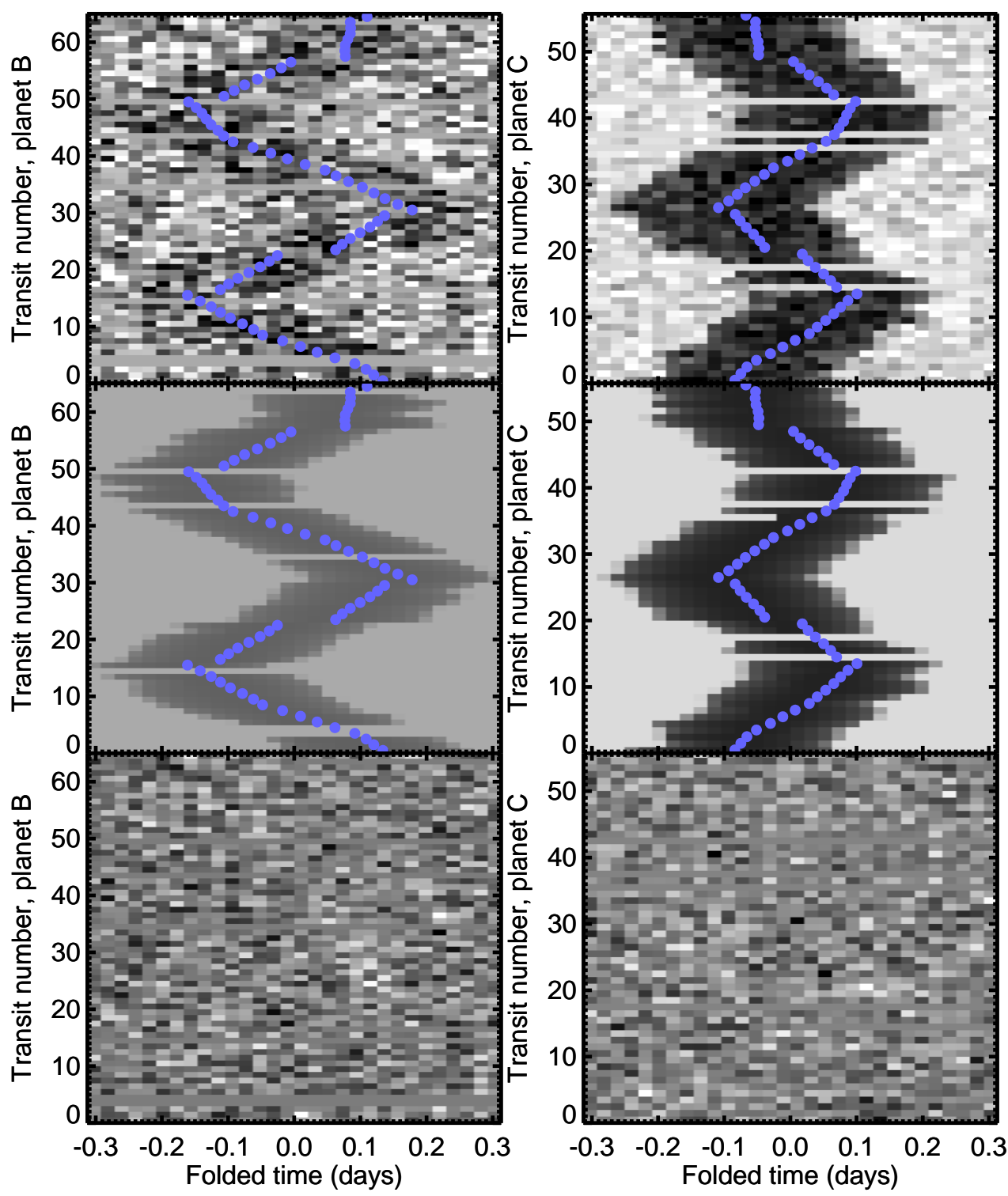

Figure S4: Plot of the transits of planet b (left) and planet c (right). Top is data; middle is best-fit photometric-dynamical model; bottom is the residuals after subtracting the model. Refer to the caption of Figure 1 for additional details. The greyscale ranges from 0.9994 (black) to 1.0001 (white) on a common scale between the top four panels. 


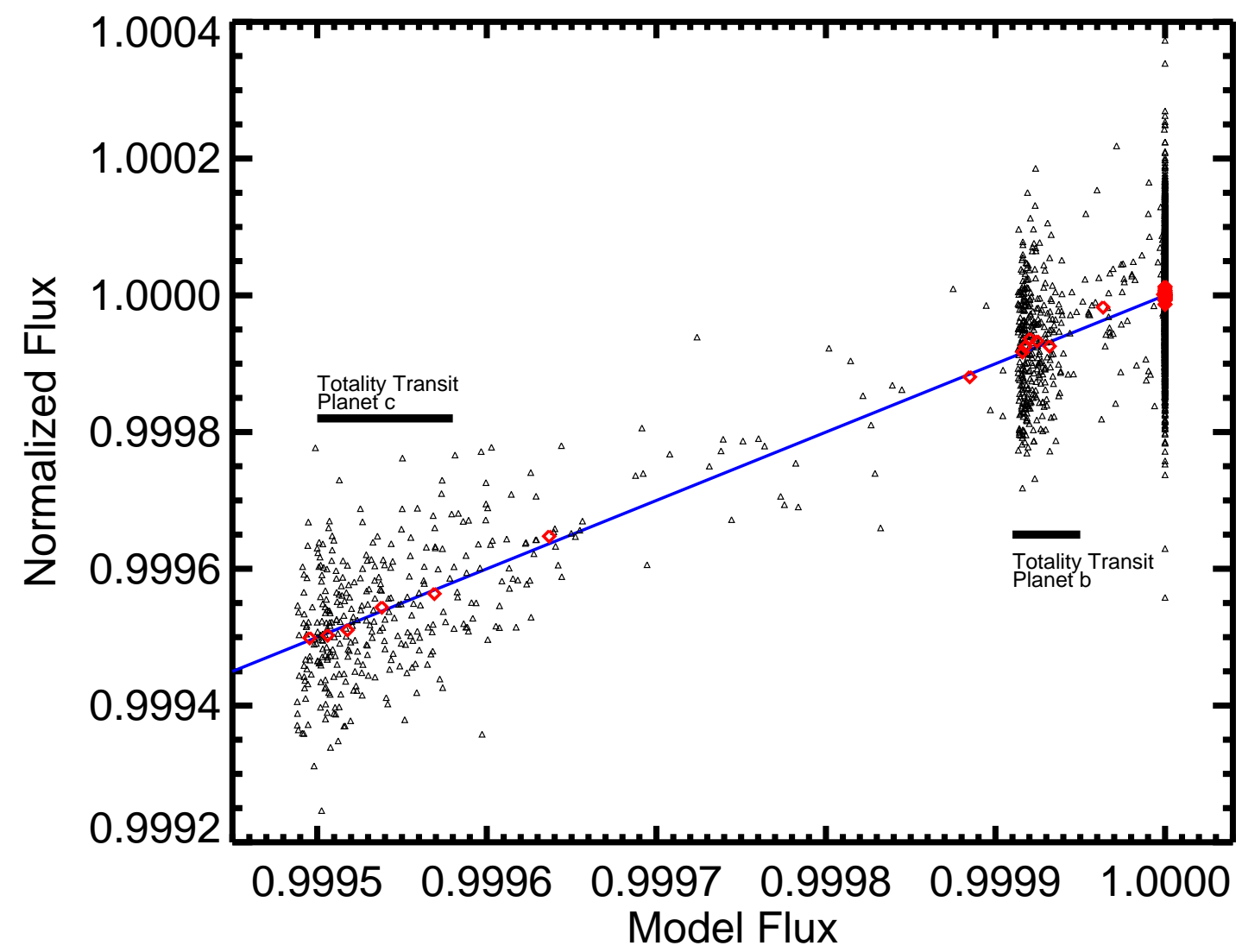

Figure S5: Correlation between the photometric-dynamical model flux and normalized Kepler photometry (i.e. after removal of a local linear fit about the transit). The red points are data, sorted in flux, averaged in bins with an equal number of data samples. The blue line denotes exact correspondence between model and data. 


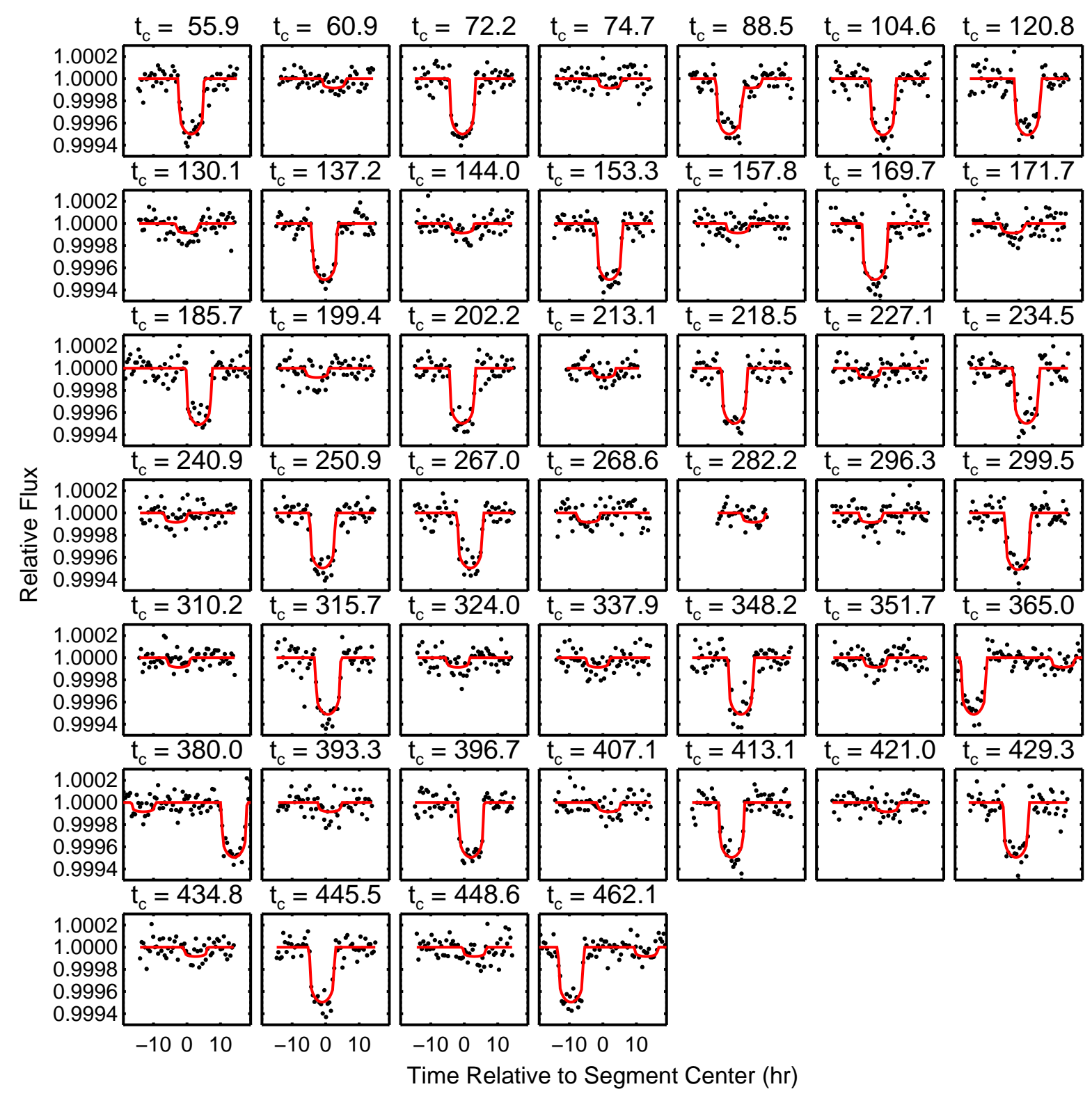

Figure S6: Long cadence Kepler data used in this analysis. Each panel shows data for a contiguous set of cadences near transits of either Planet b or Planet c. The time in listed above each panel is near the center time of each panel less 2,454,900 (BJD). The red curve is the best-fitting photometric-dynamical model. The time and flux axis is the same in all panels. 


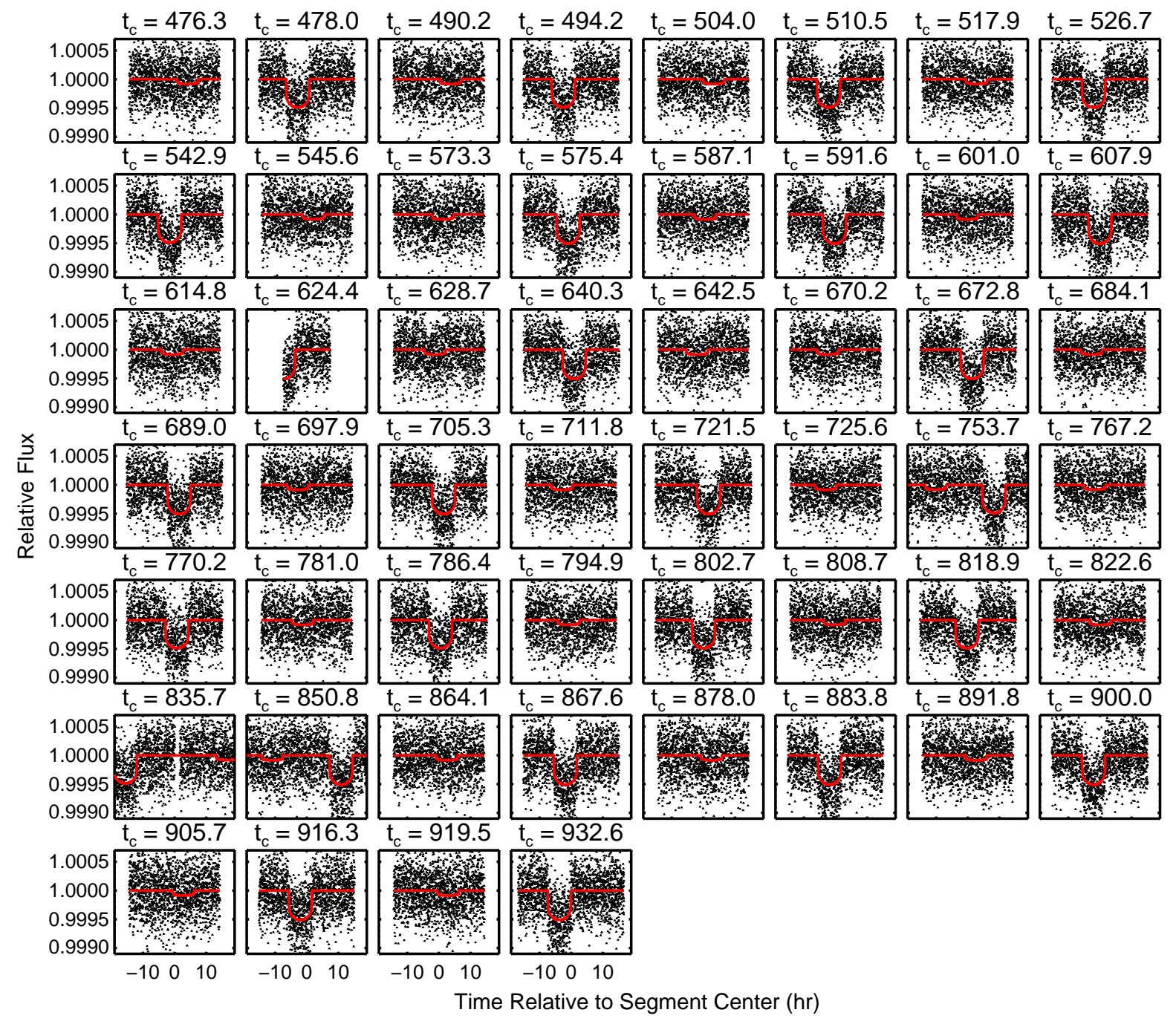

Figure S7: Short cadence Kepler data used in this analysis. Each panel shows data for a contiguous set of cadences near transits of either Planet b or Planet c. The time in listed above each panel is near the center time of each panel less 2,454,900 (BJD). The red curve is the best-fitting photometric-dynamical model. The time and flux axis is the same in all panels (but larger in flux than Figure $\mathbf{S 6}$ in order to account for the larger relative error in the short cadence data). 
observation of a long effective chain length in each parameter (as determined from the chain autocorrelation).

\subsubsection{Characteristics of the parameter posterior}

The parameter values and derived values reported in Tables S6, S7, beside the best-fit values (see $\$ 2.2 .5$ ), were found by computing the $15.8 \%, 50 \%, 84.2 \%$ levels of the cumulative distribution of the marginalized posterior for each parameter. Figure S8 shows two-parameter joint distributions between all parameters. This figure is meant to highlight the qualitative features of the posterior as opposed to providing quantitative ranges. The numbers in that figure correspond to the model parameters in Table $\mathbf{S 6}$ with the same number listed as "Index." Some non-linear degeneracies still remain in the form of "bananas" shown in the joint distribution plane, but these appear to have been properly traversed and mixed according to the convergence criteria listed above.

Figure S9 shows the posterior distribution in the eccentricity and argument of pericenter planes (this figure is discussed further in \$5). A number of arguments are permitted, however, only small eccentricities are allowed. At 95\% confidence, $e_{b}<0.039$ and $e_{c}<0.033$.

The three-dimensional "mutual" inclination between the planets' orbits, which may be defined as an osculating term at the reference epoch as

$$
\cos I=\sin i_{b} \sin i_{c} \cos \Delta \Omega+\cos i_{c} \cos i_{b},
$$

is constrained to within a couple of degrees. Figure S10 shows the distribution of $I$; Table S7 gives confidence intervals in the mutual inclination. The constraint on the mutual inclination derives from the lack of significant transit durations variations in the transits of planet c. To show this, we examined the long term variation of the sky-plane inclinations of each planet based on a draw of 100 initial conditions from our posterior (short term evolution of the posterior is described further in $\S$ (4). The inclinations show circulation of a forced inclination about a free inclination. We isolated and fitted the secular component (over the time scale of our observations) so as to compute the transit duration variation due to the secular change for planet $\mathrm{c}$ as a function of mutual inclination. We plot this for our 100 initial conditions in Figure $\mathbf{S 1 1}$. This figure shows a clear correlation between transit duration change and mutual inclination. For a mutual inclination of $\approx 2.5$ degrees - at the tail of our marginalized posterior distribution in that parameter (see Figure $\underline{\mathrm{S} 10}$ ) - the transit duration changes by about 5 minutes over the roughly 900 days of observation or approximately 0.1 minutes/epoch. We compared this rate with what can be constrained from the data: A simple transit model fit to individual transit epochs of planet $\mathrm{c}$ shows that the measured uncertainty in duration is approximately 10 minutes at each epoch. Fitting a linear model to the durations of 50 transits of $\mathrm{c}$ (the number that was observed), assuming zero transit duration variation, would yield a 1-sigma uncertainty in the 


\begin{tabular}{|c|c|c|c|c|c|}
\hline Index & Parameter Name & Best-fit & $50 \%$ & $15.8 \%$ & $84.2 \%$ \\
\hline & Mass parameters & & & & \\
\hline 0 & Mean Density, $\rho_{\star}\left(\mathrm{g} \mathrm{cm}^{-3}\right)$ & 0.3531 & 0.3511 & -0.0052 & +0.0053 \\
\hline 1 & Mass sum ratio, $q_{+}\left(\times 10^{5}\right)$ & 3.40 & 3.51 & -0.14 & +0.24 \\
\hline 2 & $\begin{array}{l}\text { Planetary mass ratio, } q_{p} \\
\text { Eccentricity parameters }\end{array}$ & 0.553 & 0.550 & -0.009 & +0.010 \\
\hline 4 & Cosine Difference, $h_{-}$ & 0.00397 & 0.00393 & -0.00027 & +0.00028 \\
\hline 6 & Cosine Sum, $h_{+}$ & 0.004 & 0.004 & -0.018 & +0.020 \\
\hline 10 & Sine Difference, $k_{-}$ & 0.0270 & 0.0258 & -0.0018 & +0.0015 \\
\hline 12 & $\begin{array}{l}\text { Sine Sum, } k_{+} \\
\text {Planet } b \text { Orbit }\end{array}$ & 0.027 & 0.017 & -0.030 & +0.012 \\
\hline 9 & Orbital Period, $P_{b}$ (day) & 13.83988 & 13.83989 & -0.00060 & +0.00082 \\
\hline 11 & Time of Transit, $t_{b}$ (days since $t_{0}$ ) & 10.97981 & 10.97529 & -0.00583 & +0.00548 \\
\hline 13 & $\begin{array}{l}\text { Orbital Inclination, } i_{b}(\mathrm{deg}) \\
\text { Planet c Orbit }\end{array}$ & 89.52 & 90.01 & -0.71 & +0.69 \\
\hline 3 & Orbital Period, $P_{c}$ (day) & 16.23853 & 16.23855 & -0.00054 & +0.00038 \\
\hline 5 & Time of Transit, $t_{c}$ (days since $t_{0}$ ) & 5.91174 & 5.91315 & -0.00097 & +0.00109 \\
\hline 7 & Orbital Inclination, $i_{c}(\mathrm{deg})$ & 89.76 & 89.98 & -0.53 & +0.54 \\
\hline 8 & $\begin{array}{l}\text { Relative Nodal Longitude, } \Delta \Omega(\mathrm{deg}) \\
\text { Radius Parameters }\end{array}$ & 0.25 & 0.06 & -1.54 & +1.48 \\
\hline 15 & Stellar Radius, $R_{\star}\left(R_{\odot}\right)$ & 1.619 & 1.626 & -0.019 & +0.019 \\
\hline 16 & b Radius Ratio, $R_{b} / R_{\star}$ & 0.00857 & 0.00838 & -0.00017 & +0.00016 \\
\hline 17 & c Radius Ratio, $R_{c} / R_{\star}$ & 0.02079 & 0.02076 & -0.00018 & +0.00018 \\
\hline 14 & $\begin{array}{l}\text { Linear Limb Darkening Parameter, } u \\
\text { Relative Contamination, } F_{\text {cont }} / F_{\star}(\times 100)\end{array}$ & 0.446 & 0.456 & -0.024 & +0.024 \\
\hline & Quarter 1 & 1.41 & 1.50 & -3.10 & +3.27 \\
\hline & Quarter 2 & -0.20 & -0.33 & -2.46 & +2.46 \\
\hline & Quarter 3 & 1.75 & 0.77 & -2.53 & +2.61 \\
\hline & Quarter 4 & -0.98 & -0.22 & -2.81 & +2.79 \\
\hline & Quarter 5 & 2.34 & 1.97 & -2.56 & +2.64 \\
\hline & Quarter 6 & 3.43 & 2.97 & -2.32 & +2.39 \\
\hline & Quarter 7 & & & 0 (fixed) & \\
\hline & Quarter 8 & -0.02 & -1.41 & -2.31 & +2.37 \\
\hline & Quarter 9 & 3.71 & 3.76 & -2.27 & +2.28 \\
\hline & Quarter 10 & -0.72 & -2.29 & -2.02 & +2.12 \\
\hline & Noise Parameters & & & & \\
\hline & Long Cadence Relative Width, $\sigma_{\mathrm{LC}}\left(\times 10^{5}\right)$ & 7.89 & 7.87 & -0.10 & +0.11 \\
\hline & Short Cadence Relative Width, $\sigma_{\mathrm{SC}}\left(\times 10^{5}\right)$ & 33.692 & 33.681 & -0.077 & +0.078 \\
\hline
\end{tabular}

Table S6: Model parameters as defined in the text. The reference epoch is $t_{0}=2,454,950$ (BJD). 


\begin{tabular}{|c|c|c|c|c|}
\hline Parameter & Best-fit & $50 \%$ & $15.8 \%$ & $84.2 \%$ \\
\hline \multicolumn{5}{|l|}{ Planetary Bulk Properties } \\
\hline Mass of Planet $\mathrm{b}, M_{b}\left(M_{\oplus}\right)$ & 4.28 & 4.45 & -0.27 & +0.33 \\
\hline Mass of Planet c, $M_{c}\left(M_{\oplus}\right)$ & 7.73 & 8.08 & -0.46 & +0.60 \\
\hline Radius of Planet $\mathrm{b}, R_{b}\left(R_{\oplus}\right)$ & 1.512 & 1.486 & -0.035 & +0.034 \\
\hline Radius of Planet c, $R_{c}\left(R_{\oplus}\right)$ & 3.669 & 3.679 & -0.054 & +0.054 \\
\hline Density of Planet $\mathrm{b}, \rho_{b}\left(\mathrm{~g} \mathrm{~cm}^{-3}\right)$ & 6.80 & 7.46 & -0.59 & +0.74 \\
\hline Density of Planet $\mathrm{c}, \rho_{c}\left(\mathrm{~g} \mathrm{~cm}^{-3}\right)$ & 0.860 & 0.891 & -0.046 & +0.066 \\
\hline Planetary Density Ratio, $\rho_{b} / \rho_{c}$ & 7.91 & 8.35 & -0.46 & +0.51 \\
\hline Planet b to Star Density Ratio, $\rho_{b} / \rho_{\star}$ & 19.3 & 21.2 & -1.6 & +2.1 \\
\hline Planet c to Star Density Ratio, $\rho_{c} / \rho_{\star}$ & 2.44 & 2.53 & -0.12 & +0.19 \\
\hline Surface Gravity of Planet $\mathrm{b}, g_{b}\left(\mathrm{~m} \mathrm{~s}^{-2}\right)$ & 18.3 & 19.7 & -1.3 & +1.7 \\
\hline Surface Gravity of Planet c, $g_{c}\left(\mathrm{~m} \mathrm{~s}^{-2}\right)$ & 5.62 & 5.83 & -0.28 & +0.42 \\
\hline Escape Velocity of Planet $\mathrm{b}, v_{\mathrm{esc}, b}\left(\mathrm{~km} \mathrm{~s}^{-1}\right)$ & 18.80 & 19.34 & -0.57 & +0.73 \\
\hline Escape Velocity of Planet c, $v_{\mathrm{esc}, c}\left(\mathrm{~km} \mathrm{~s}^{-1}\right)$ & 16.22 & 16.56 & -0.42 & +0.58 \\
\hline \multicolumn{5}{|l|}{ Orbital Properties } \\
\hline Semimajor Axis of Planet $\mathrm{b}, a_{b}(\mathrm{AU})$ & 0.1151 & 0.1153 & -0.0015 & +0.0015 \\
\hline Semimajor Axis of Planet c, $a_{c}(\mathrm{AU})$ & 0.1280 & 0.1283 & -0.0016 & +0.0016 \\
\hline$e_{b} \cos \omega_{b}+\left(a_{c} / a_{b}\right) e_{c} \cos \omega_{c}$ & 0.004 & 0.004 & -0.019 & +0.021 \\
\hline$e_{b} \cos \omega_{b}-\left(a_{c} / a_{b}\right) e_{c} \cos \omega_{c}$ & 0.00444 & 0.00439 & -0.00029 & +0.00031 \\
\hline$e_{b} \sin \omega_{b}+\left(a_{c} / a_{b}\right) e_{c} \sin \omega_{c}$ & 0.027 & 0.017 & -0.032 & +0.013 \\
\hline$e_{b} \sin \omega_{b}-\left(a_{c} / a_{b}\right) e_{c} \sin \omega_{c}$ & 0.02698 & 0.02664 & -0.00139 & +0.00094 \\
\hline Mutual Orbital Inclination, $I$ (deg) & 0.35 & 1.20 & -0.63 & +0.79 \\
\hline Orbital Velocity of Planet b, $2 \pi a_{b} / P_{b}\left(\mathrm{~km} \mathrm{~s}^{-1}\right)$ & 90.4 & 90.7 & -1.2 & +1.2 \\
\hline Orbital Velocity of Planet c, $2 \pi a_{c} / P_{c}\left(\mathrm{~km} \mathrm{~s}^{-1}\right)$ & 85.8 & 86.0 & -1.1 & +1.1 \\
\hline Mutual Hill Radius, $R_{H},\left(\frac{q_{+}}{24}\right)^{1 / 3}\left(a_{b}+a_{c}\right)(\mathrm{AU})$ & 0.002730 & 0.002769 & -0.000054 & +0.000067 \\
\hline \multicolumn{5}{|l|}{ Transit Parameters } \\
\hline Radius Ratio of Planet $\mathrm{b}, R_{b} / R_{\star}$ & 0.00857 & 0.00838 & -0.00017 & +0.00016 \\
\hline Radius Ratio of Planet $\mathrm{c}, R_{c} / R_{\star}$ & 0.02079 & 0.02076 & -0.00018 & +0.00018 \\
\hline Impact Parameter of Planet $\mathrm{b}, b_{b} / R_{\star}$ & 0.13 & -0.00 & -0.18 & +0.19 \\
\hline Impact Parameter of Planet $\mathrm{c}, b_{c} / R_{\star}$ & 0.07 & 0.00 & -0.16 & +0.16 \\
\hline Transit Velocity of Planet $\mathrm{b}, v_{b} / R_{\star}\left(\right.$ day $\left.^{-1}\right)$ & 7.129 & 7.079 & -0.104 & +0.043 \\
\hline Transit Velocity of Planet $\mathrm{c}, v_{c} / R_{\star}\left(\right.$ day $\left.^{-1}\right)$ & 6.579 & 6.541 & -0.087 & +0.035 \\
\hline Transit Duration of Planet b (hr) & 6.74 & 6.78 & -0.11 & +0.09 \\
\hline Transit Duration of Planet c (hr) & 7.430 & 7.443 & -0.020 & +0.020 \\
\hline Transit Ingress/Egress Duration of Planet b (min) & 3.490 & 3.455 & -0.086 & +0.122 \\
\hline Transit Ingress/Egress Duration of Planet c (min) & 9.12 & 9.21 & -0.14 & +0.27 \\
\hline Temperature Scaling of Planet $\mathrm{b}, \sqrt{R_{\star} / 2 a_{b}}$ & 0.18087 & 0.18104 & -0.00045 & +0.00045 \\
\hline Temperature Scaling of Planet $\mathrm{c}, \sqrt{R_{\star} / 2 a_{c}}$ & 0.17149 & 0.17165 & -0.00043 & +0.00043 \\
\hline
\end{tabular}

Table S7: Derived Parameters. The reference epoch is $t_{0}=2,454,950$ (BJD). 
linear model slope of 0.1 minutes/epoch. The near equivalence between these two numbers - the 1-sigma constraint from the observed durations with assumed-flat transit duration variation and the numerically estimated rate assuming the 1-sigma value of mutual inclinations - demonstrates our claim.

\section{Bulk composition of the Kepler-36 planets}

\subsection{Bayesian Constraints on Kepler-36b's Bulk Composition}

In this section we elaborate briefly on the analysis constraining Kepler-36b's composition.

Following the Bayesian approach outlined in (83), we account for the (correlated) observational uncertainties in the planet mass and radius in a rigorous way when constraining the planet composition. We adopt a uniform prior on the planet mass and a flat prior on planet composition. The likelihood function (which quantifies how well a specific combination of planet mass and composition fit the observed data) is set by the joint $M_{p}-R_{p}$ posterior derived from MCMC fits to the transit timing variations and transit light curves (illustrated in Figure 3 in the main text). We calculate planet radii at a given mass and composition using the interior structure model of $(84,85)$.

We first explore a rocky planet scenario for Kepler-36b, in which the planet consists of a metal iron core surrounded by a $\mathrm{Mg}_{0.9} \mathrm{Fe}_{0.1} \mathrm{SiO}_{3}$ silicate mantle. Our main conclusion is that Kepler-36b could be a rocky planet with an Earth-like composition (with $\sim 30 \%$ of its mass in an iron core, and $\sim 70 \%$ of its mass in a silicate mantle). Marginalizing over planet mass, the iron core mass fraction of Kepler-36b is constrained. We find median and $68 \%$ credible intervals of $M_{\text {core }} / M_{p}=0.29_{-0.10}^{+0.11}$.

We turn to the possibility that Kepler-36b harbors a water envelope that contributes to its transit radius. We follow a similar Bayesian analysis approach as described above, but now consider a three component model for the planet structure (iron core, silicate mantle, water envelope) with a flat prior on the fraction of the planet mass in each layer. In this three component composition model, inherent degeneracies come to bare on the composition constraints; many compositions agree with a single planet mass and radius. The mass of a water envelope on Kepler- $36 \mathrm{~b}$ is less than $23 \%$ of the total planet mass, at $68 \%$ Bayesian confidence. The most water rich composition within the $68 \%$ credible region that have iron abundances below the (86) constraints from silicate collisional stripping simulations is $13 \% \mathrm{H}_{2} \mathrm{O}$.

Finally, considering the possibility for a H/He gas layer on Kepler-36b, we find that Kepler$36 \mathrm{~b}$ could have no more than $1 \%$ of its mass in solar composition $\mathrm{H} / \mathrm{He}$ layer. 


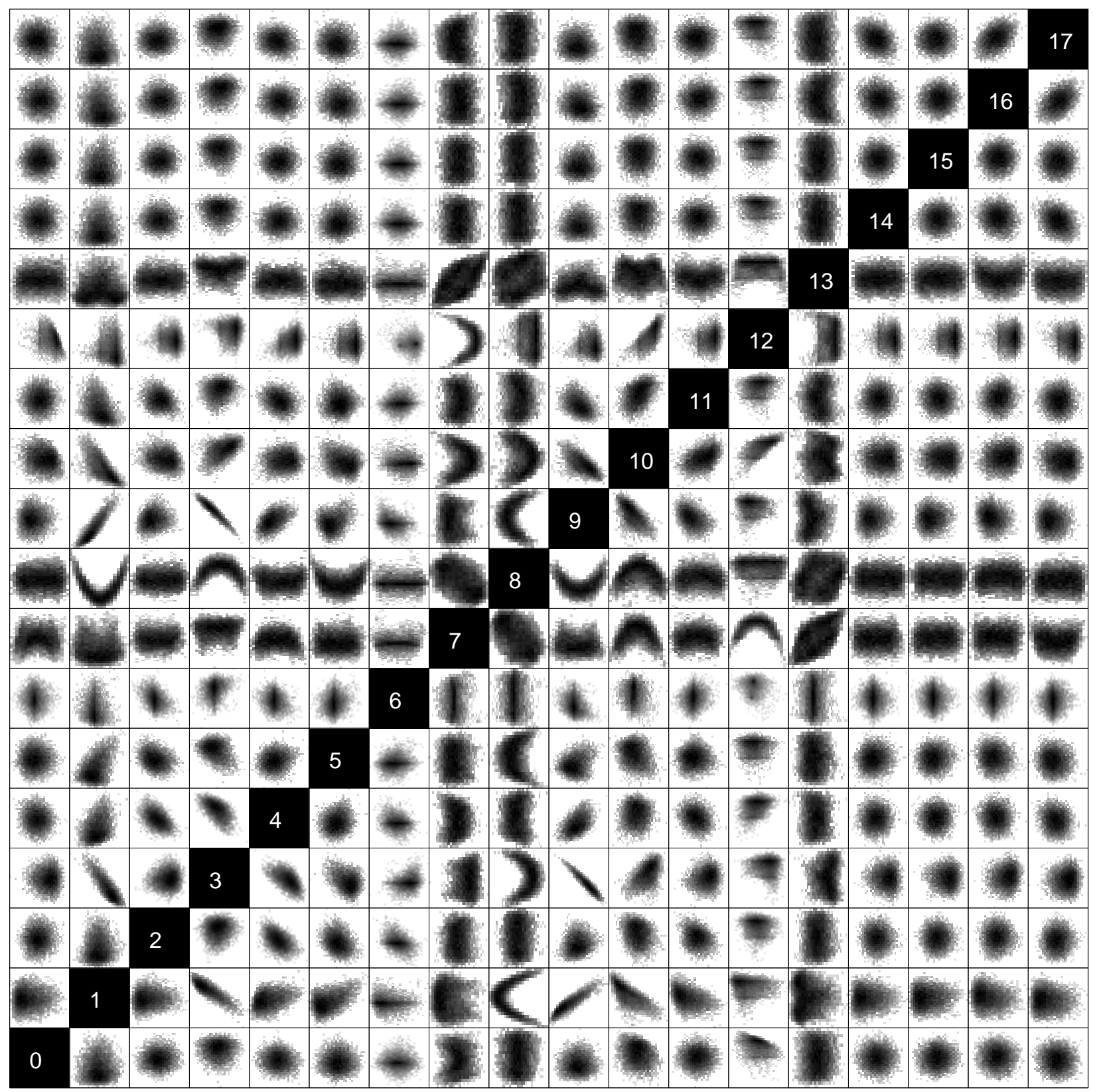

Figure S8: Two-parameter joint posterior distributions of the primary model parameters (excluding contamination parameters; see \$2.2.3). The densities are plotted logarithmically in order to elucidate the nature of the parameter correlations. The indices listed along the diagonal indicate which parameter is associated with the corresponding row and column. The parameter name corresponding to a given index is indicated in Table S6 in the "Index" column. 

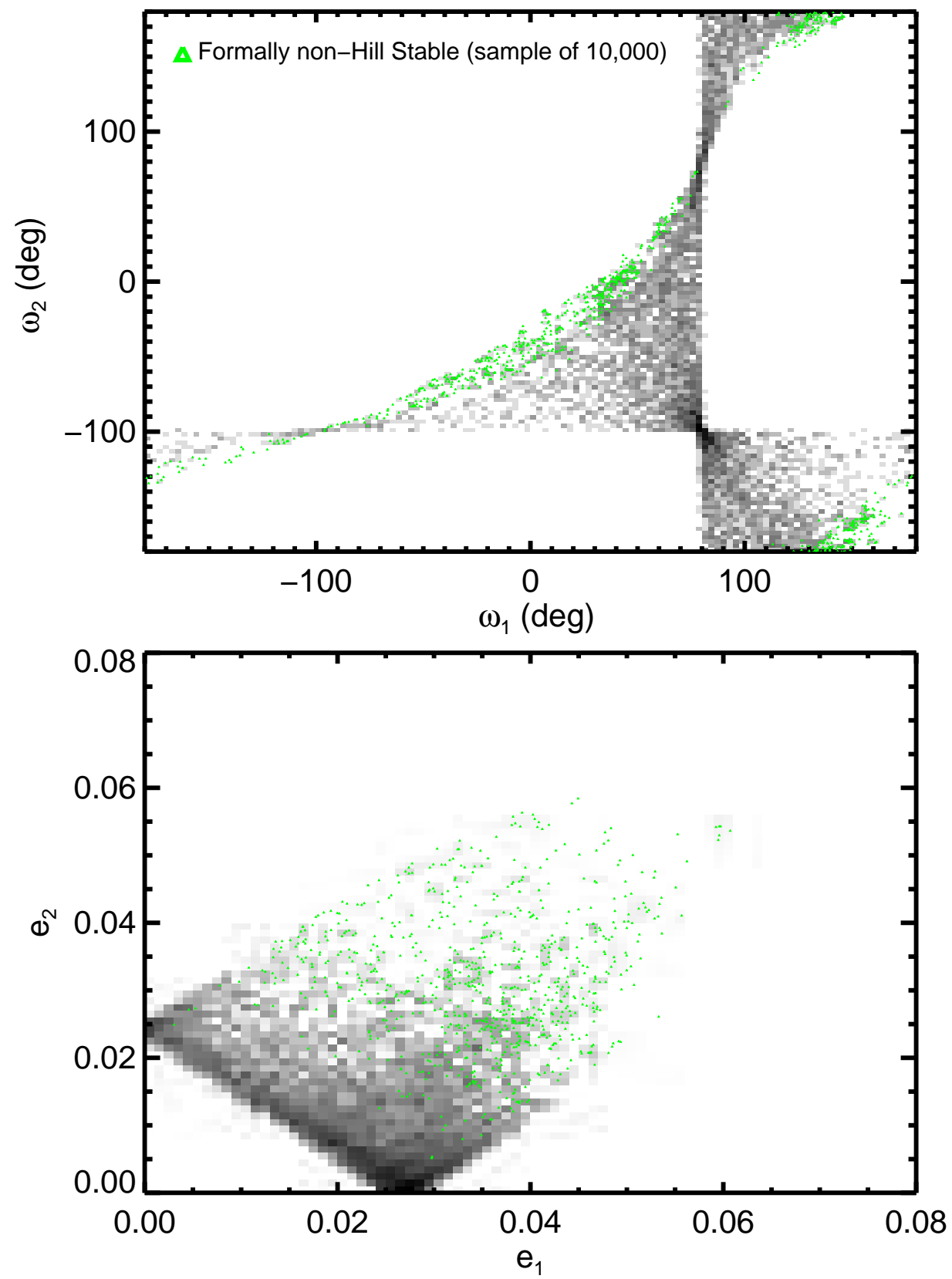

Figure S9: Posterior distributions in the eccentricity and argument of pericenter planes. 


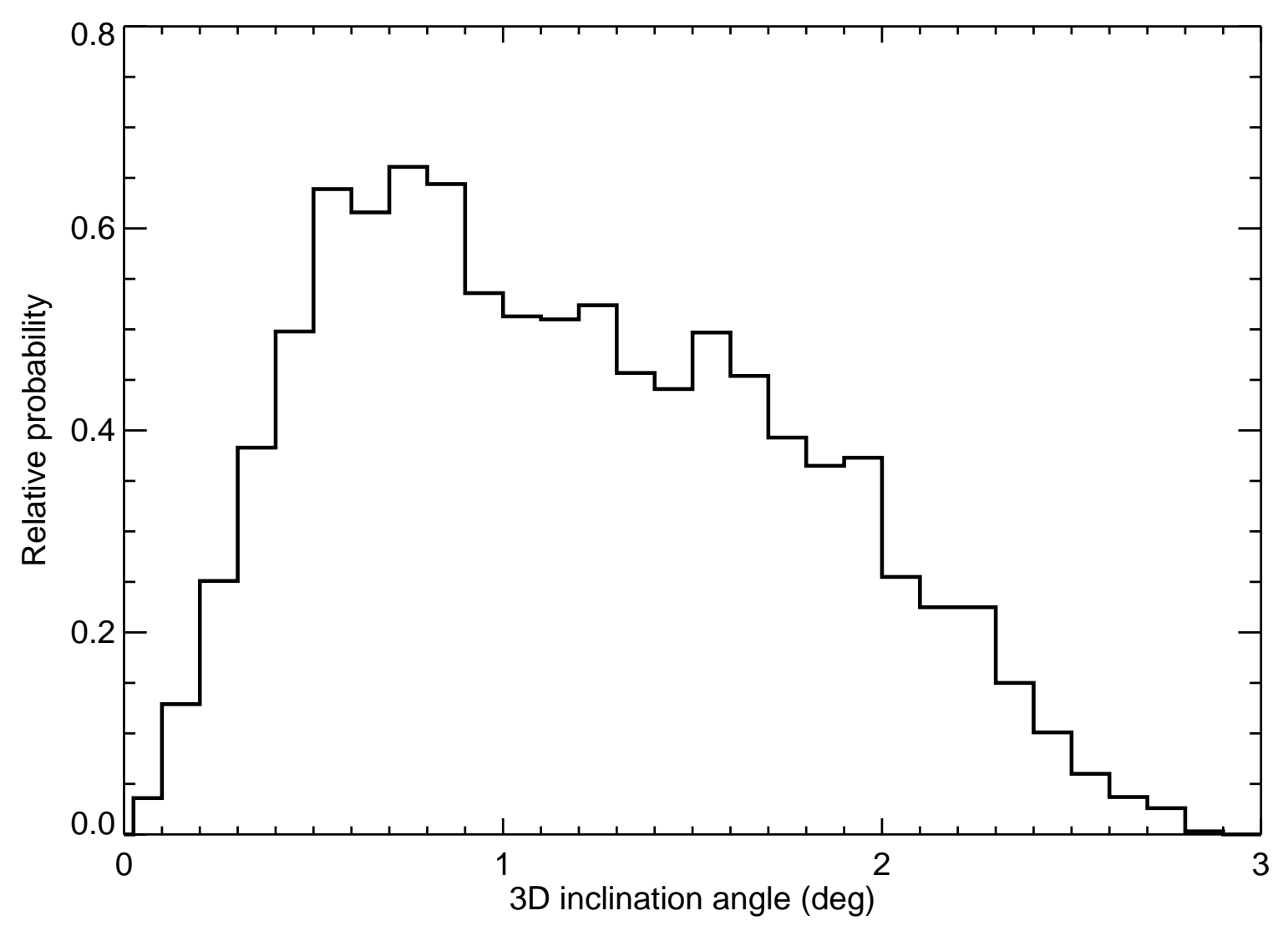

Figure S10: Posterior distribution of the mutual inclination, $I$, of the planetary orbits. 


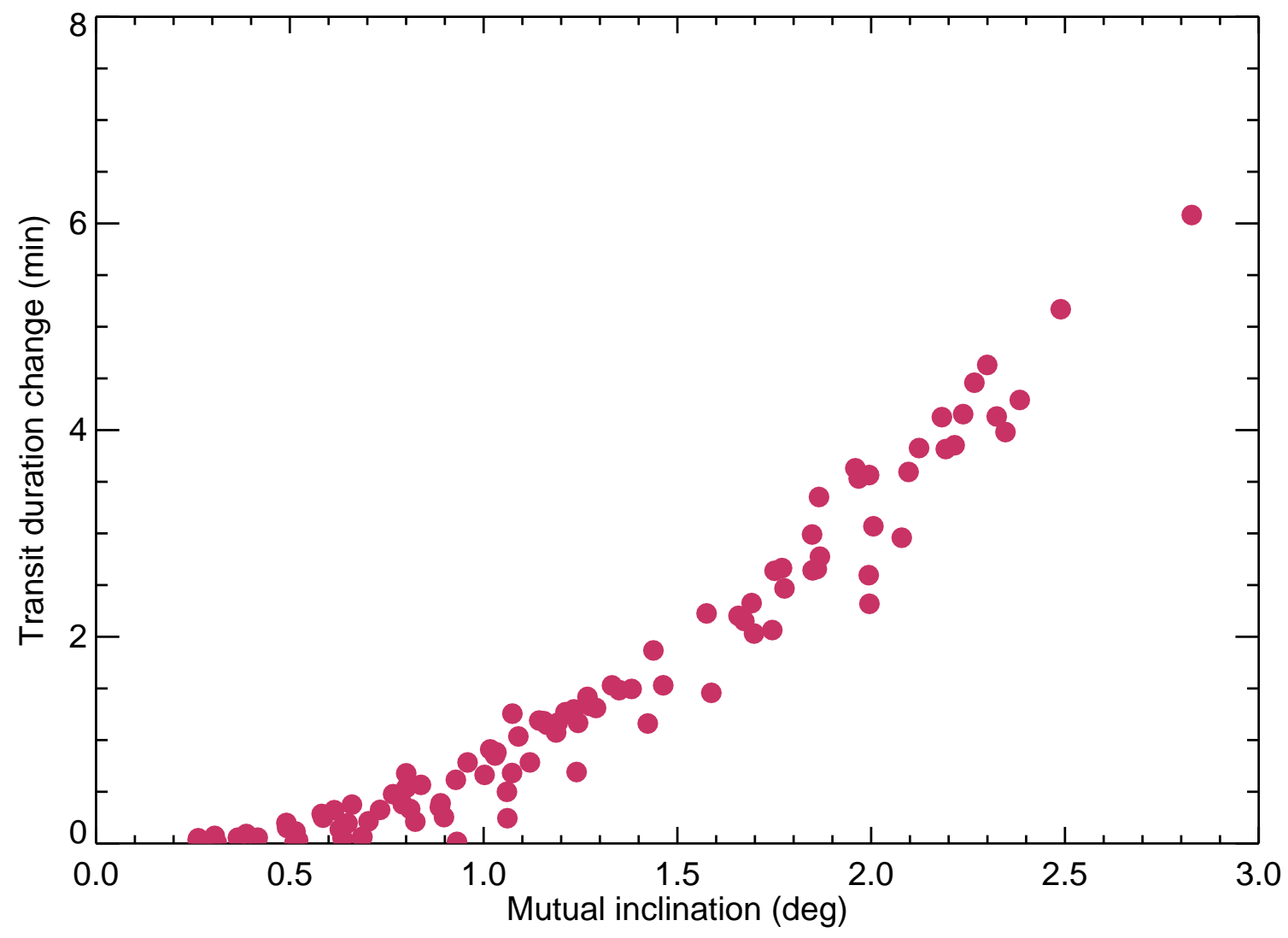

Figure S11: The change in the transit duration (secular component) over the span of observations as a function of mutual inclination for a random sample of 100 initial conditions from the posterior. 


\subsection{Bayesian constraints on Kepler-36c's composition}

Kepler-36c has a low enough mean density that it must have a hydrogen-rich envelope (a water envelope is not puffy enough). In the main text (and elaborated here in the supplementary online material) we have presented evolution calculations to constrain the $\mathrm{H} / \mathrm{He}$ envelope mass. Assuming a hot-start formation process and passive cooling at the planet's current orbital separation, tight constraints on Kepler-36c's interior entropy and H/He mass obtain. The formation mechanism and the full dynamical and insolation histories of the Kepler-36 planets are not known, however.

In this section we complement the planet cooling calculations with a more expansive investigation of parameter space, to illustrate the dependence of the planet compositional inference on the intrinsic luminosity of the planet. In cases where the planet history is more complicated than the isolated cooling calculations have assumed, a systematic offset in the prediction of $L_{p}(t)$ could result. The Bayesian contours in Figures $\mathbf{S 1 2}$ and $\mathbf{S 1 3}$ offer Kepler-36c composition constraints as a function of the planet intrinsic luminosity.

We consider two scenarios for the composition of the planet's heavy-element interior: a rocky (iron and silicate) interior (Figure $\underline{\mathrm{S12}}$ ), and an ice-rock interior consisting of $60 \% \mathrm{H}_{2} \mathrm{O}$ and $40 \%$ rock by mass (Figure $\underline{\mathrm{S} 13}$ ). In both scenarios we consider a range of iron to silicate mass ratios, and assume a uniform prior on the mass fraction within each compositional layer in the planet. Four different choices of Kepler-36c's intrinsic luminosity are shown for illustration: $L_{p} / M_{p}=10^{-13} \mathrm{~W} \mathrm{~kg}^{-1}$, a very low intrinsic luminosity to set strong upper limits on the mass of $\mathrm{H} / \mathrm{He} ; L_{p} / M_{p}=10^{-11.5} \mathrm{~W} \mathrm{~kg}^{-1} \approx \mathrm{L}_{\text {radio }} / \mathrm{M}_{\mathrm{p}}$, the expected luminosity from the decay of radiogenic isotopes at $6.8 \mathrm{Gyr}$ assuming bulk Earth abundances; $L_{p} / M_{p}=10^{-10.5} \mathrm{~W} \mathrm{~kg}^{-1} \approx 10 \mathrm{~L}_{\text {radio }} / \mathrm{M}_{\mathrm{p}}$; and $L_{p} / M_{p}=10^{-9.5} \mathrm{~W} \mathrm{~kg}^{-1} \approx 100 \mathrm{~L}_{\text {radio }} / \mathrm{M}_{\mathrm{p}}$. For low-mass planets with gas layers, the intrinsic luminosity of the planet can have an important effect on the "puffiness" of the gas layer. The H/He envelope mass fraction inferred for Kepler-36c is smaller for higher entropy, high $L_{p}$ cases. The hot-start evolution calculations favor the higher luminosity scenarios.

The Bayesian composition constraint contours in Figures $\underline{\mathbf{S 1 2}}$ and $\mathbf{S 1 3}$ provide a lower bound on the uncertainties in Kepler-36c's interior composition and $\mathrm{H} / \mathrm{He}$ envelope mass at a single snapshot of planet intrinsic luminosity. Marginalizing over the planet cooling history to account for the uncertainty in $L_{p}(t)$ will further broaden these composition constraints. Uncertainties quoted in the cooling calculation results are not credible intervals like those presented in Figures $\mathbf{S 1 3}$ and $\mathbf{S 1 2}$, but are instead estimated by varying one parameter $\left(M_{p}, R_{p}, C_{p}\right.$, age, incident flux) at a time and adding in quadrature. The heavier computational burden of the evolution calculation currently precludes a full Bayesian analysis of the composition uncertainties. Ultimately, future work will incorporate cooling calculations in the Bayesian approach to determine priors on $L_{p}(t)$ and the planet interior entropy. 


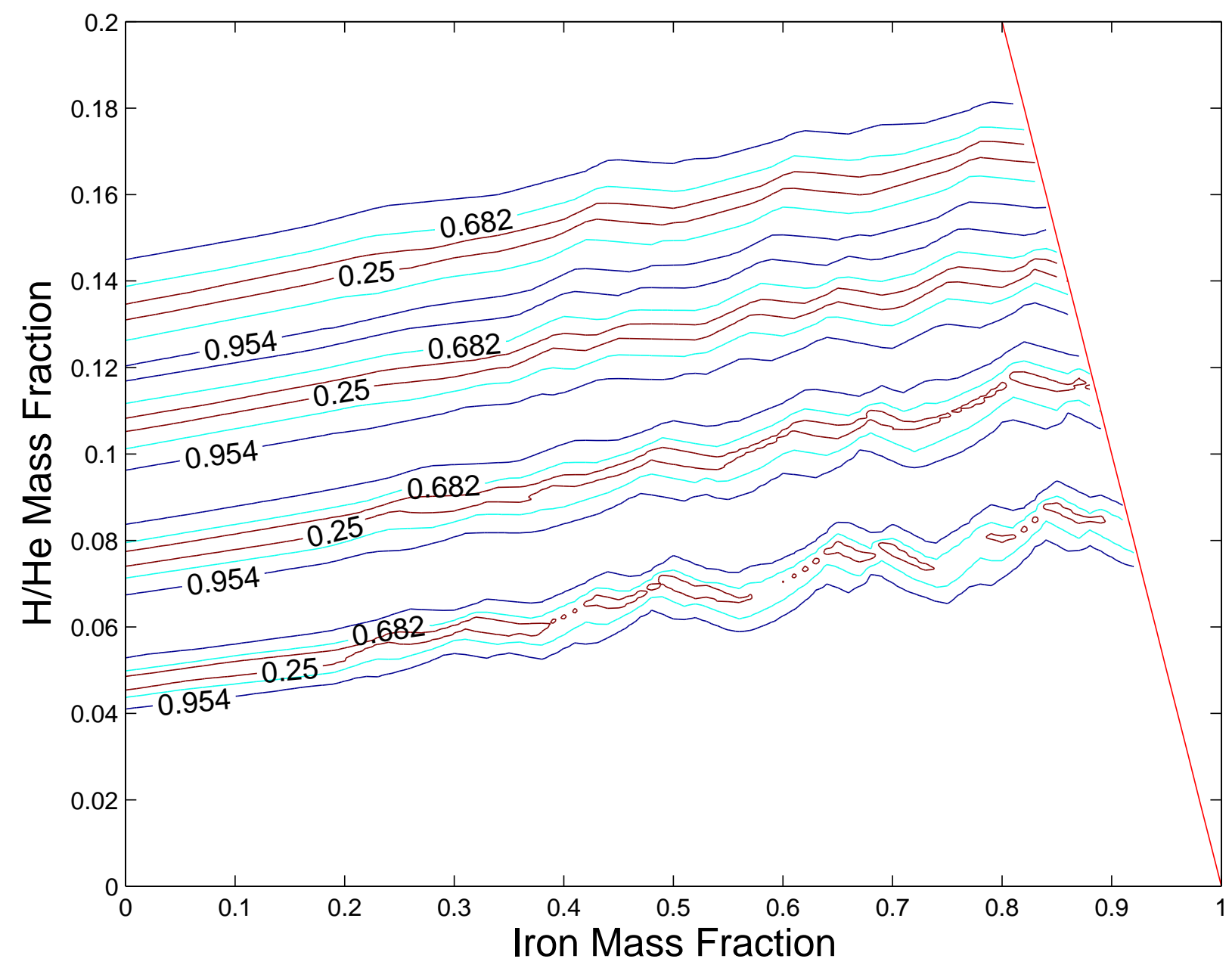

Figure S12: Bayesian constraints on the mass of a solar composition envelope surrounding Kepler-36c assuming a rocky interior. The contours plotted are lines of constant probability labeled with a Bayesian confidence value indicating the degree of belief (between 0 and 1) that the true composition of the planet falls within the contour (given the assumed priors). A rocky heavy element interior comprised of an metal iron core and silicate mantle is assumed. The Bayesian contours in Figures $[\mathbf{S 1 2}$ and $[\mathbf{S 1 3}$ are not marginalized over the planet evolution history, but instead show composition constraints for four different choices of Kepler-36c's intrinsic luminosity. From high to low $\mathrm{H} / \mathrm{He}$ content (low to high $L_{p}$ ) the values shown are: $L_{p} / M_{p}=10^{-13} \mathrm{~W} \mathrm{~kg}^{-1}$, a very low intrinsic luminosity to set strong upper limits on the mass of $\mathrm{H} / \mathrm{He} ; L_{p} / M_{p}=10^{-11.5} \mathrm{~W} \mathrm{~kg}^{-1} \approx \mathrm{L}_{\text {radio }} / \mathrm{M}_{\mathrm{p}}$, the expected luminosity from the decay of radiogenic isotopes at $6.8 \mathrm{Gyr}$ assuming bulk Earth abundances; $L_{p} / M_{p}=10^{-10.5} \mathrm{~W} \mathrm{~kg}^{-1} \approx 10 \mathrm{~L}_{\text {radio }} / \mathrm{M}_{\mathrm{p}} ;$ and $L_{p} / M_{p}=10^{-9.5} \mathrm{~W} \mathrm{~kg}^{-1} \approx 100 \mathrm{~L}_{\text {radio }} / \mathrm{M}_{\mathrm{p}}$. 


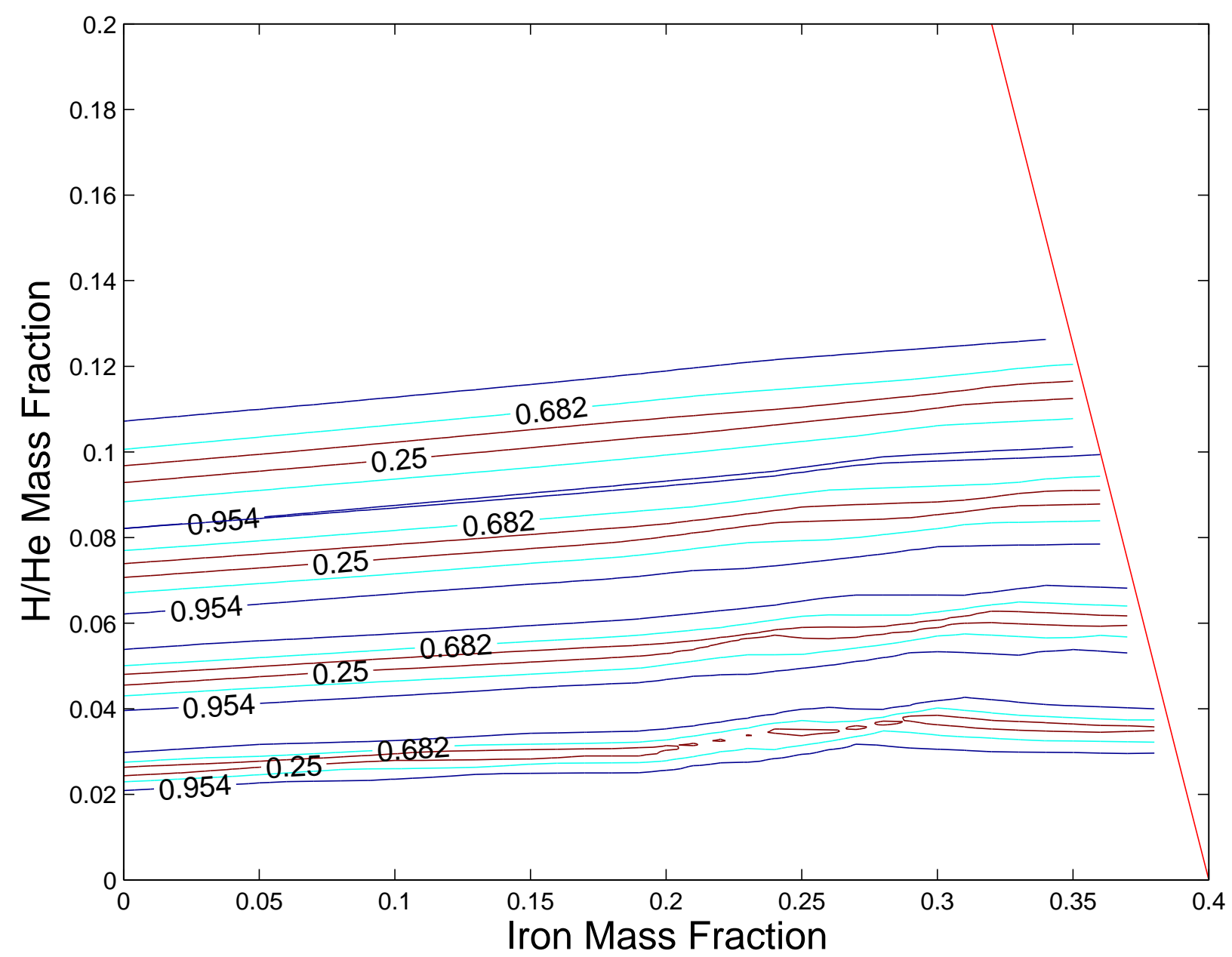

Figure S13: Bayesian constraints on the mass of a solar composition envelope surrounding Kepler-36c assuming an ice-rock interior. This figure is identical to Figure \$12, but assumes the heavy element interior of Kepler-36c is $60 \% \mathrm{H}_{2} \mathrm{O}$ and $40 \%$ rock by mass. The iron to silicate ratio abundance is still allowed to vary. 


\subsection{Constraints on Kepler-36c considering thermal evolution}

Kepler-36c is low density, substantially less dense than pure water. As a result, it must have a substantial Hydrogen \& Helium $(\mathrm{H} / \mathrm{He})$ envelope. In order to estimate the planet's mass fraction in $\mathrm{H} / \mathrm{He}$, we use a coupled thermal evolution and mass loss code (87). These models are based on the model used in (88), which has been adapted for modeling Super-Earths following the methods of (89). By fully modeling the thermal evolution of the planet's interior, we are able to get a much more precise estimate of the the planet's interior structure. Models that only compute an instantaneous structure are forced to vary the intrinsic luminosity of the planet over several orders of magnitude, which introduces large errors in the composition.

In particular, the model assumes an adiabatic $\mathrm{H} / \mathrm{He}$ interior structure that initially has a large entropy from formation. We then track the planet's radius up to the present day as it cools and contracts. In general, the planet's radius is insensitive to the initial entropy choice by 100 Myr. The bottom of the $\mathrm{H} / \mathrm{He}$ adiabat then attaches to an isothermal core with a Earth-like rock/iron ratio. Meanwhile, the radiative top of the atmosphere also becomes isothermal once this intrinsic flux from this adiabat equals the incident flux at the top of the atmosphere. We find cooling rates at the top of the atmosphere, by interpolating between models of the intrinsic flux computed on a grid of gravity, Teff, and entropy. These cooling rates were computed using the self-consistent, non-gray radiative transfer models described in (29), assuming cloud-free 50x solar opacities.

For the rocky core we use the ANEOS olivine equation of state (EOS) (90) for the rock and SESAME FeEOS (91) for the iron. For the H/He envelope we use SCVH (92) and for $\mathrm{H}_{2} \mathrm{O}$ we use H2O-REOS (93).

Finally, we model the cooling of the rocky core. As the $\mathrm{H} / \mathrm{He}$ adiabat cools and contracts, the isothermal core needs to cool as well. However, because this rock/iron core has a nonzero heat capacity, the presence of a large core will slow down the cooling of the atmosphere. Although this effect is negligible for gas giants, it becomes dominant for Super-Earths. Models that neglect the cooling of the core will predict interiors that are too cold and over predict amount of volatiles needed to match the observed radius. We vary the heat capacity of the rock from $0.5-1.0 \mathrm{~J} / \mathrm{K} / \mathrm{g}$ as in (89). We also included radiogenic heating in the rocky core, assuming earth like abundances of $\mathrm{U}$, Th, and $\mathrm{K}$.

In order to determine the current composition of Kepler-36c, we ran thermal evolution models without mass loss and adjusted the mass of the core until we matched the observed radius at the current age. We then determined the uncertainty in the current composition due to the observed uncertainties in the planet's mass, radius, age, and incident flux along with the theoretical uncertainties in the atmospheric albedo, the heat capacity and the rock/iron ratio of the core. We varied the albedo from $0-0.8$ and the iron fraction from pure rock to the maximum possible iron fraction from collisional stripping (20). The dominant sources of error were the 
planet radius, the iron fraction, and the heat capacity of the core, all others were negligible. Finally, we were able to get a estimate of the current composition of Kepler-36c of $8.6 \pm_{1.3}^{1.4} \%$ $\mathrm{H} / \mathrm{He}$, assuming a water-free interior.

We also explored the possibility of a water-rich interior for Kepler-36c. To do this we inserted a water layer equal in mass to the rocky core in between the rock/iron core and the $\mathrm{H} / \mathrm{He}$ envelope. From the thermal evolution, we find that this water layer should be in the molecular and ionic fluid phases and not in high pressure ices. We find that for a water-rich, or "Neptune-like" composition, Kepler-36c needs $1.6 \pm 0.4 \%$ of its mass in $\mathrm{H} / \mathrm{He}$.

Despite the large density contrast seen today, it is possible both planets could have been volatile rich in the past. Due their high incident fluxes, both planets are vulnerable to XUV driven atmospheric escape of light gases $(94,95)$. Although it is likely rocky today, we find that Kepler-36b could have been as much as $40 \% \mathrm{H} / \mathrm{He}$ when the system was $100 \mathrm{Myr}$ old and the stellar XUV flux was $\sim 200$ times higher (96). With similar models, we find that Kepler-36c would have been $35 \pm 9 \% \mathrm{H} / \mathrm{He}$, assuming a dry interior.

\section{Short-term behavior and secular characteristics of Kepler-36}

\subsection{Evolution of the best-fit model}

The orbital elements show oscillatory patterns as a function of time. Figure $\$ 14$ shows the eccentricity vectors plotted versus time. The expected variation in eccentricity is $\approx 0.01$, which is indeed the case for the best-fit model. The eccentricity variation of the smaller planet is larger by a factor of the mass ratio, $\approx 1.8$, which is expected due to conservation of momentum during the conjunctions. The orbital elements stay approximately constant in between conjunctions, then show a jump as they pass through conjunction. As the orientations of the conjunctions circulate with time, the orbital elements oscillate. When the components of the eccentricity vectors are plotted versus one another, Figure S15, the pattern becomes rather intricate. The orbital elements remain nearly constant between conjunctions creating the knots in each petal. During conjunction the planets undergo the largest change in orbital elements, forming the wiggles connecting the knots. The conjunctions drift in inertial space over $\approx 5$ conjunctions; however, the planets have slightly different orbital elements after 5 conjunctions, so the petals drift and eventually form the entire "flower."

The planet orbits precess with time; Figure $\mathbf{5 1 6}$ shows the inclination angle and sky nodal angle for one of the best-fit models. The timescale of precession in this case is $\approx 2.5 \times 10^{4}$ days. 


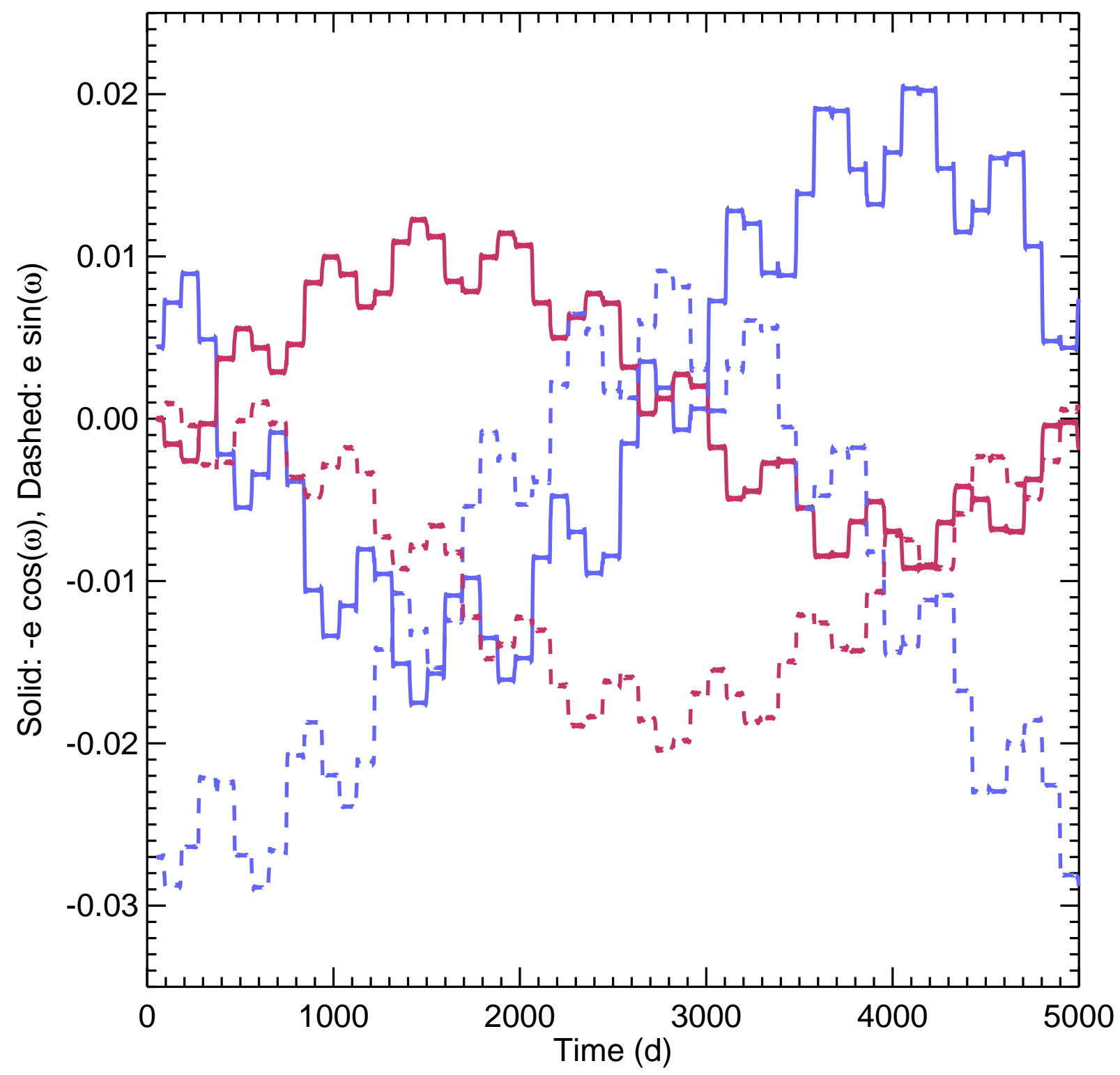

Figure S14: Plot of eccentricity vectors for the two planets as a function of time. Kepler-36b is plotted in blue, while Kepler-36c is plotted in red. 


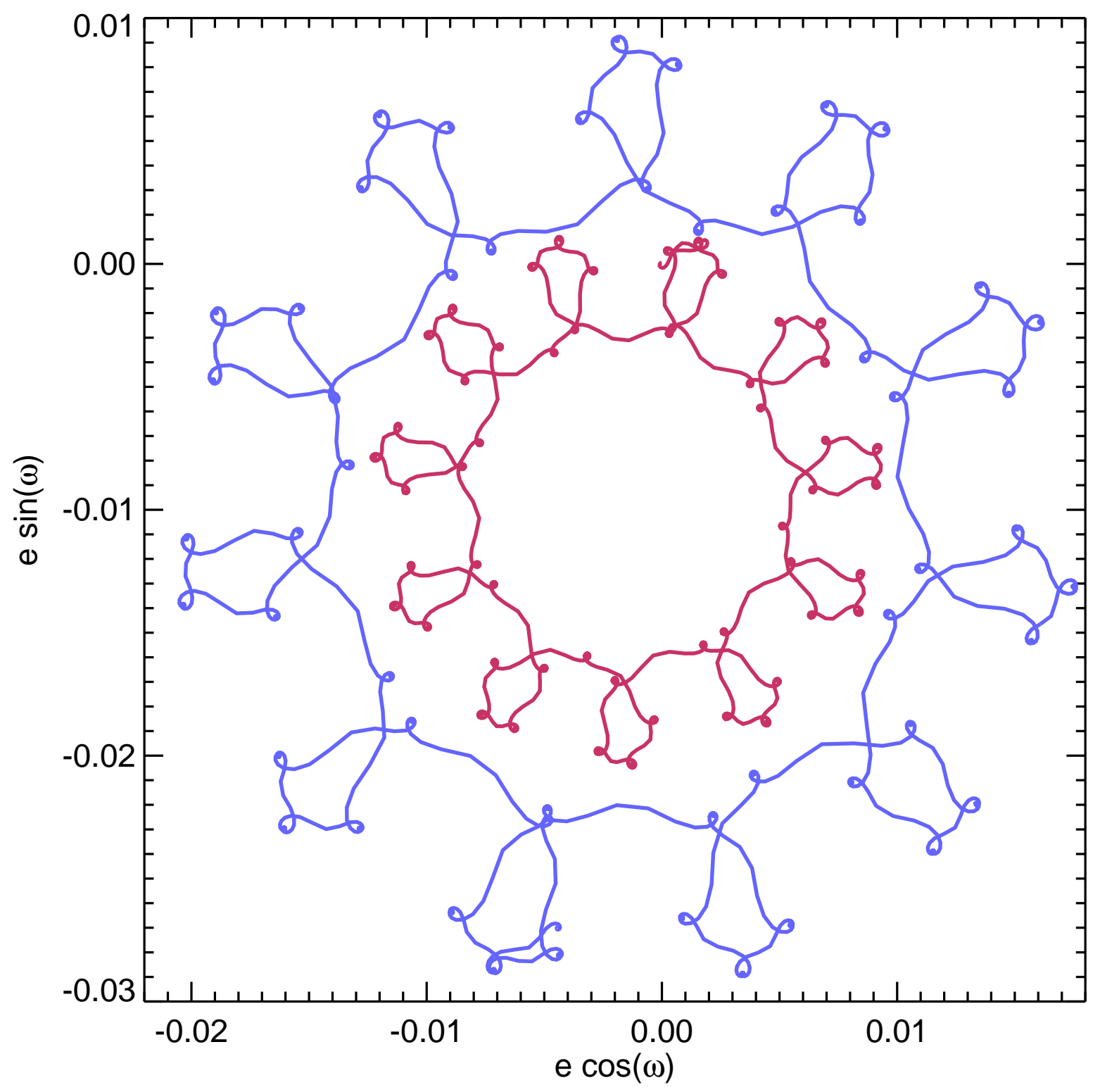

Figure S15: Plot of the two components of the eccentricity vector versus one another. Kepler$36 \mathrm{~b}$ is plotted in blue, while Kepler-36c is plotted in red. 


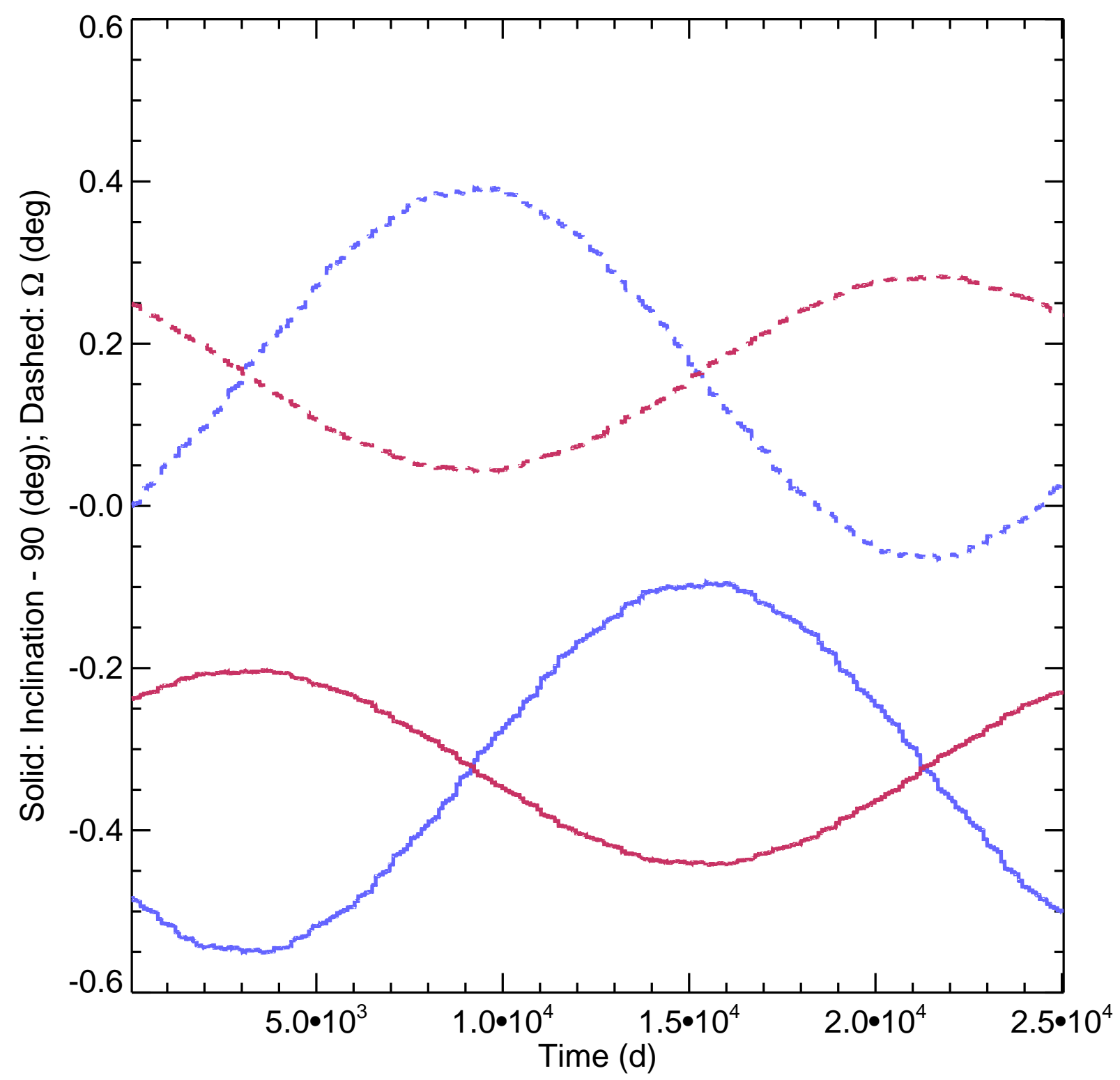

Figure S16: Plot of inclination angles for the two planets and nodal angles as a function of time. Kepler-36b is plotted in blue, while Kepler-36c is plotted in red. 


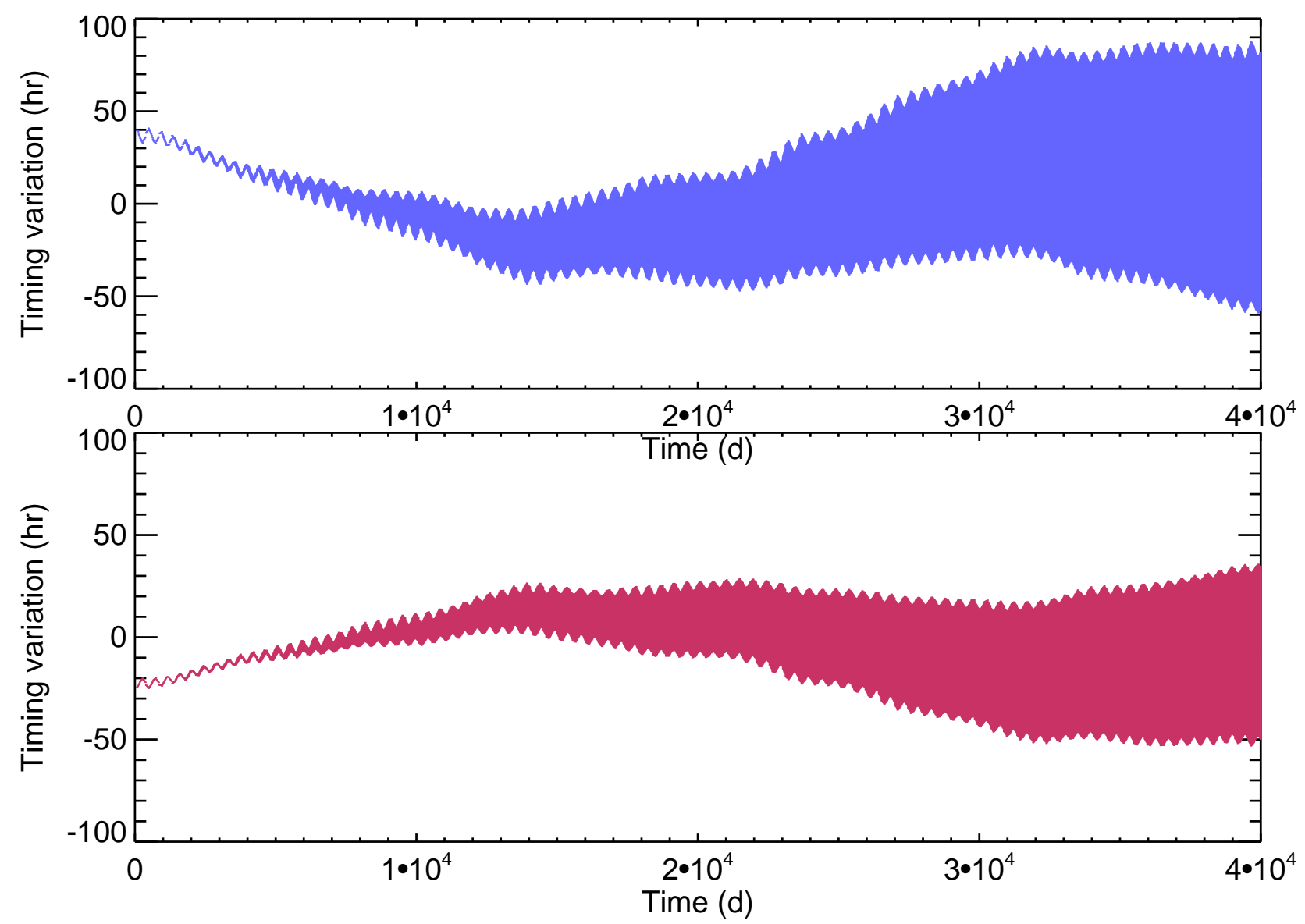

Figure S17: Predicted transit timing variations based on the models drawn from the posterior distribution. The range indicates the $68 \%$ confidence range predicted for planet $b$ (blue) and planet c (red).

\subsection{Predicted Ephemerides and transit parameters}

Tables $\mathbf{S} 8$ and $\mathbf{S 9}$ provide predicted times of transit, impact parameters and duration for the next year (starting near May 1, 2012) with errors. Figure S18 plots predictions over a much longer time range after subtracting a linear model. Figure $[\mathbf{S 1 8}$ shows the variations in the transit durations during that same time.

\section{$5 \quad$ Long-term stability of solutions}

To assess the long term stability of the system, we selected 10,001 random draws from the posterior distribution of parameters (masses, initial positions, and initial velocities) and integrated each of these for $6.845 \times 10^{5}$ years (over 15 million orbits of planet c). We used a symplectic $n$ - 


\begin{tabular}{|l|l|l|l|}
\hline Epoch, $E$ & $T_{\text {transit }}-\left(\left\langle T_{0}\right\rangle+E\langle P\rangle\right)(\mathrm{hr})$ & Impact Parameter & Duration (hr) \\
\hline 0 & $0.98 \pm 0.22$ & $0.130 \pm 0.078$ & $6.783 \pm 0.077$ \\
1 & $0.10 \pm 0.22$ & $0.130 \pm 0.078$ & $6.783 \pm 0.077$ \\
2 & $-0.32 \pm 0.22$ & $0.130 \pm 0.078$ & $6.809 \pm 0.077$ \\
3 & $-0.47 \pm 0.21$ & $0.130 \pm 0.078$ & $6.807 \pm 0.077$ \\
4 & $-0.67 \pm 0.21$ & $0.130 \pm 0.078$ & $6.808 \pm 0.077$ \\
5 & $-0.78 \pm 0.21$ & $0.130 \pm 0.078$ & $6.808 \pm 0.077$ \\
6 & $-0.87 \pm 0.21$ & $0.130 \pm 0.078$ & $6.808 \pm 0.077$ \\
7 & $-1.01 \pm 0.21$ & $0.130 \pm 0.078$ & $6.808 \pm 0.077$ \\
8 & $-1.23 \pm 0.21$ & $0.130 \pm 0.078$ & $6.807 \pm 0.077$ \\
9 & $-0.10 \pm 0.19$ & $0.129 \pm 0.079$ & $6.856 \pm 0.078$ \\
10 & $0.02 \pm 0.19$ & $0.129 \pm 0.079$ & $6.855 \pm 0.078$ \\
11 & $0.15 \pm 0.19$ & $0.129 \pm 0.079$ & $6.856 \pm 0.078$ \\
12 & $0.37 \pm 0.19$ & $0.129 \pm 0.079$ & $6.856 \pm 0.078$ \\
13 & $0.59 \pm 0.20$ & $0.129 \pm 0.079$ & $6.856 \pm 0.078$ \\
14 & $0.75 \pm 0.21$ & $0.129 \pm 0.079$ & $6.855 \pm 0.078$ \\
15 & $0.85 \pm 0.22$ & $0.129 \pm 0.079$ & $6.855 \pm 0.078$ \\
16 & $2.31 \pm 0.23$ & $0.129 \pm 0.081$ & $6.872 \pm 0.079$ \\
17 & $1.78 \pm 0.25$ & $0.129 \pm 0.081$ & $6.872 \pm 0.079$ \\
18 & $1.28 \pm 0.26$ & $0.129 \pm 0.081$ & $6.873 \pm 0.079$ \\
19 & $0.86 \pm 0.28$ & $0.129 \pm 0.081$ & $6.873 \pm 0.079$ \\
20 & $0.44 \pm 0.30$ & $0.129 \pm 0.081$ & $6.873 \pm 0.079$ \\
21 & $-0.06 \pm 0.32$ & $0.129 \pm 0.081$ & $6.872 \pm 0.079$ \\
22 & $-0.59 \pm 0.34$ & $0.129 \pm 0.081$ & $6.873 \pm 0.079$ \\
23 & $-0.51 \pm 0.37$ & $0.128 \pm 0.081$ & $6.853 \pm 0.078$ \\
24 & $-1.48 \pm 0.38$ & $0.128 \pm 0.081$ & $6.853 \pm 0.078$ \\
25 & $-2.38 \pm 0.39$ & $0.128 \pm 0.081$ & $6.853 \pm 0.078$ \\
\hline
\end{tabular}

Table S8: Predicted ephemerides, impact parameters and durations for Kepler-36b for one year. In the table, times are given relative to a linear ephemeris with $\left\langle T_{0}\right\rangle=2456049.39050$ (BJD) and $\langle P\rangle=13.85940$ days. Epoch $E=0$ is near May 1, 2011. 


\begin{tabular}{|l|l|l|l|}
\hline Epoch, $E$ & $T_{\text {transit }}-\left(\left\langle T_{0}\right\rangle+E\langle P\rangle\right)(\mathrm{hr})$ & Impact Parameter & Duration (hr) \\
\hline 0 & $-0.81 \pm 0.05$ & $0.096 \pm 0.062$ & $7.438 \pm 0.019$ \\
1 & $-0.18 \pm 0.05$ & $0.096 \pm 0.062$ & $7.438 \pm 0.019$ \\
2 & $0.19 \pm 0.04$ & $0.095 \pm 0.062$ & $7.423 \pm 0.020$ \\
3 & $0.33 \pm 0.04$ & $0.095 \pm 0.062$ & $7.424 \pm 0.021$ \\
4 & $0.46 \pm 0.03$ & $0.095 \pm 0.062$ & $7.423 \pm 0.021$ \\
5 & $0.53 \pm 0.03$ & $0.095 \pm 0.062$ & $7.423 \pm 0.021$ \\
6 & $0.62 \pm 0.03$ & $0.095 \pm 0.062$ & $7.424 \pm 0.021$ \\
7 & $0.77 \pm 0.03$ & $0.095 \pm 0.062$ & $7.424 \pm 0.021$ \\
8 & $0.11 \pm 0.03$ & $0.093 \pm 0.063$ & $7.395 \pm 0.024$ \\
9 & $0.03 \pm 0.04$ & $0.093 \pm 0.063$ & $7.395 \pm 0.024$ \\
10 & $-0.07 \pm 0.05$ & $0.093 \pm 0.063$ & $7.394 \pm 0.024$ \\
11 & $-0.23 \pm 0.06$ & $0.093 \pm 0.063$ & $7.394 \pm 0.024$ \\
12 & $-0.36 \pm 0.07$ & $0.093 \pm 0.063$ & $7.395 \pm 0.024$ \\
13 & $-0.42 \pm 0.09$ & $0.093 \pm 0.063$ & $7.395 \pm 0.024$ \\
14 & $-1.32 \pm 0.10$ & $0.092 \pm 0.064$ & $7.384 \pm 0.027$ \\
15 & $-0.93 \pm 0.11$ & $0.092 \pm 0.064$ & $7.384 \pm 0.027$ \\
16 & $-0.59 \pm 0.12$ & $0.092 \pm 0.064$ & $7.384 \pm 0.027$ \\
17 & $-0.28 \pm 0.14$ & $0.092 \pm 0.064$ & $7.384 \pm 0.027$ \\
18 & $0.06 \pm 0.15$ & $0.092 \pm 0.064$ & $7.384 \pm 0.027$ \\
19 & $0.45 \pm 0.17$ & $0.092 \pm 0.064$ & $7.384 \pm 0.027$ \\
20 & $0.47 \pm 0.18$ & $0.091 \pm 0.064$ & $7.396 \pm 0.028$ \\
21 & $1.17 \pm 0.19$ & $0.091 \pm 0.064$ & $7.396 \pm 0.028$ \\
\hline
\end{tabular}

Table S9: Predicted ephemerides, impact parameters and durations for Kepler-36c for one year. In the table, times are given relative to a linear ephemeris with $\left\langle T_{0}\right\rangle=2456044.91461$ (BJD) and $\langle P\rangle=16.22407$ days. Epoch $E=0$ is near April 27, 2011. 


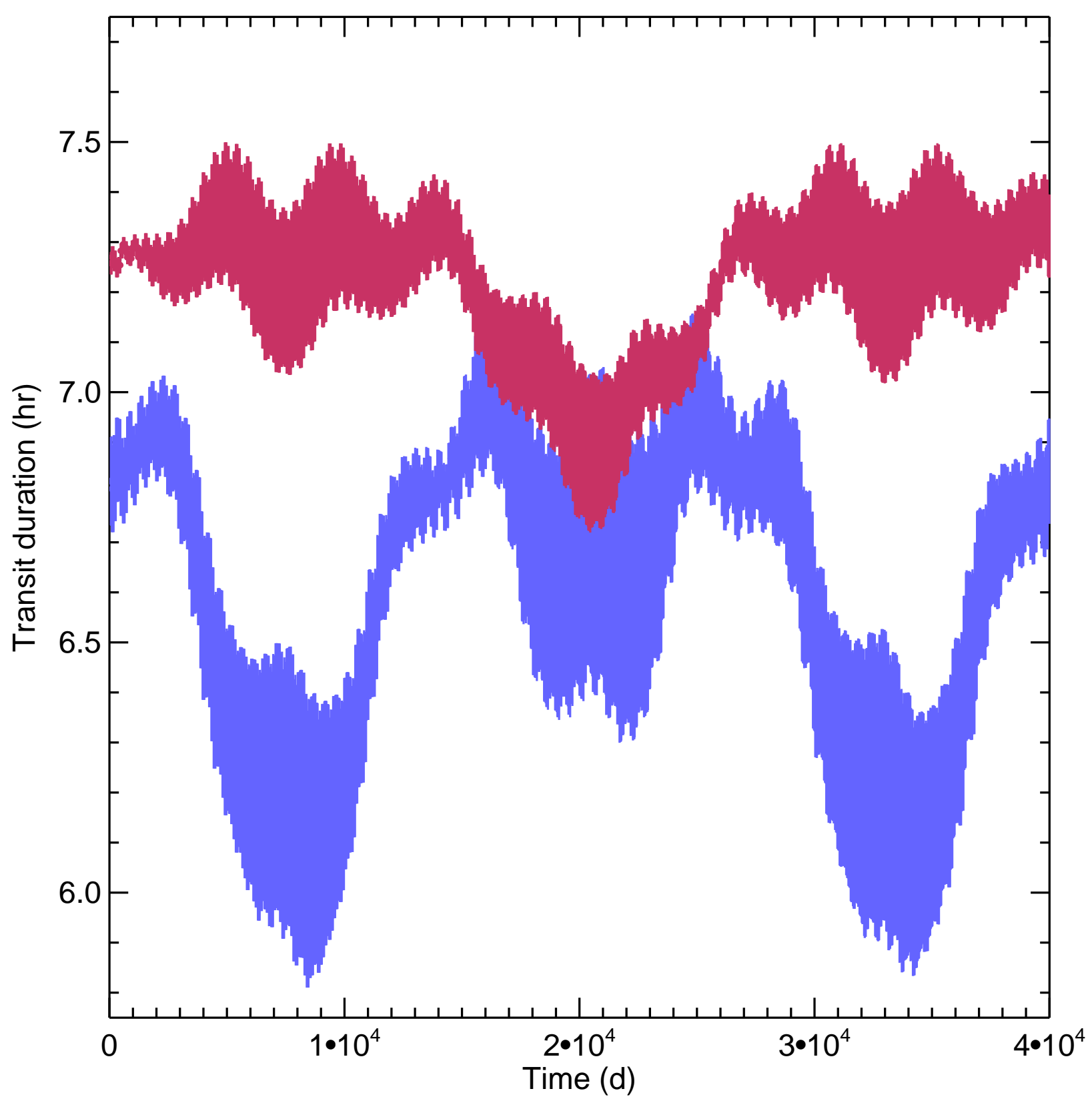

Figure S18: Predicted transit duration variations based on the models drawn from the posterior distribution. The range indicates the $68 \%$ confidence range predicted for planet $\mathrm{b}$ (blue) and planet c (red). 
body map $(97,98)$ in Jacobi coordinates, with the third-order symplectic corrector developed by J. Chambers and reported by Wisdom (2006). In separate work we determined that for the mass ratios of Kepler-36b and c (relative to the mass of the star), the Chambers corrector provides the optimal balance between precision and computational cost.

The integrations used a time step of 0.125 days. With the symplectic corrector, the relative energy error of the integrations was typically $10^{-12}$ or smaller, with a negligible secular trend. With this time step size, the conjunctions are resolved well. Furthermore, given the degree of conservation of the total energy, we believe that this time step is small enough to integrate the orbits accurately, despite the relatively close encounters that occur. To test this, we integrated a small sample of the initial conditions with a Bulirsch-Stoer integrator and compared the result with that from the symplectic $n$-body map. We confirmed that the output of the two methods agrees for short integrations.

Out of the full set of 10,001 initial conditions, no initial configuration which satisfied the Hill criterion was found to have a close encounter, which we defined as occurring when the planets were within one Hill radius (99) of each other. We selected a random sample of 100 initial conditions and integrated these for $\sim 140$ Million years. Again, there were no close encounters.

Out of the full set of 10,001 initial conditions, 1,171 satisfied $(p / a)-(p / a)_{c r i t}<10^{-4}$. Of this subset, 313 were formally Hill stable and 858 did not satisfy the Hill criterion [ $(p / a)-$ $\left.(p / a)_{c r i t}<0,(12)\right]$. In other words, these are the initial conditions that do not, or nearly do not, satisfy the Hill stability criterion. We integrated these for $13.69 \times 10^{6}$ years, again with a time step of 0.125 days and with a close encounter threshold of $1.0 R_{H}$. No initial condition that satisfied the Hill stability criterion underwent an encounter during the integrations. This both further confirms the criterion and supports the validity of our integrations.

The majority of the 858 initial conditions that do not satisfy the Hill criterion have close encounters: 616 passed within one Hill sphere of each other during the 13.69 million years integrations. No initial conditions had a close encounter earlier than $10^{6}$ days into the integration. Although the Hill stability criterion makes no formal statement about the stability or instability of orbits that do not satisfy the criterion, Gladman (1993) (13) found that systems with small eccentricities that do not satisfy the stability criterion typically undergo close encounters on short time scales. Since the data constrain eccentricities to be relatively small, we might expect that the remaining initial conditions that do not satisfy Hill stability will undergo encounters during longer integrations. There is no pressing need to follow up on these exhaustively, though, as the goal of this stability analysis was not to understand completely the stability of every initial condition. Instead, we have established that $\sim 91 \%$ of the posterior distribution is long lived and does not undergo close encounters. Furthermore, we do not believe that the presence of dynamically unstable orbits in the posterior reflects on the stability of the best fit solution, as 
the Hill unstable portion of the posterior lies on the fringes of the distribution (see Figure S9).

Although the vast majority of the sample of initial conditions are Hill stable, this stability criterion alone does not imply that the orbits are Lagrange stable (i.e. that their semimajor axes are bounded). The condition of Lagrange stability can further constrain the posterior, just as the condition of Hill stability does. This will be explored in future work.

\section{Comparison with other planet systems}

Kepler-36 is an outlier in both separation and difference in density: it has the smallest fractional separation of any two adjacent planets with measured masses and radii, and it has the largest density contrast, save for Kepler-18b,c (Figure S20).

The planets Kepler-36 appear to be located at a border between super-Earths and miniNeptunes: Kepler-36b is the coolest super-Earth with a measured density known to date, while Kepler-36c is the hottest mini-Neptune (S21). In the figure we have indicated a suggested division between super-Earths and mini-Neptunes at a density of $3.5 \mathrm{~g} / \mathrm{cc}$; planets less than this density likely have a significant H/He envelope. Any low-density planets (i.e. mini-Neptunes) at small orbital separations should be easily detected as these have a higher transit probability and deeper transit depth. It is possible that these planets were formed here, but evaporation removed the $\mathrm{H} / \mathrm{He}$ envelopes, causing these planets to evolve towards super-Earths; we have indicated this with an arrow in the figure. However, it is quite possible that the hot mini-Neptune region will be filled in with further observations.

\section{Availability of data}

The Kepler light curve data used in this analysis can be downloaded from the Mikulski Archives for Space Telescopes at the address below by searching for 11401755 in the "Kepler ID" field:

$\backslash$ protect \vrule width0pt \protect \href $\{$ http://archive.stsci.edu/kepler/data

The spectra used in this analysis have been provided (as ASCII text files) as an attachment to this supplement;

McDonald co-added spectrum :

1223269 s2 $\cdot$ txt

HIRES spectrum:

1223269 s3.txt 


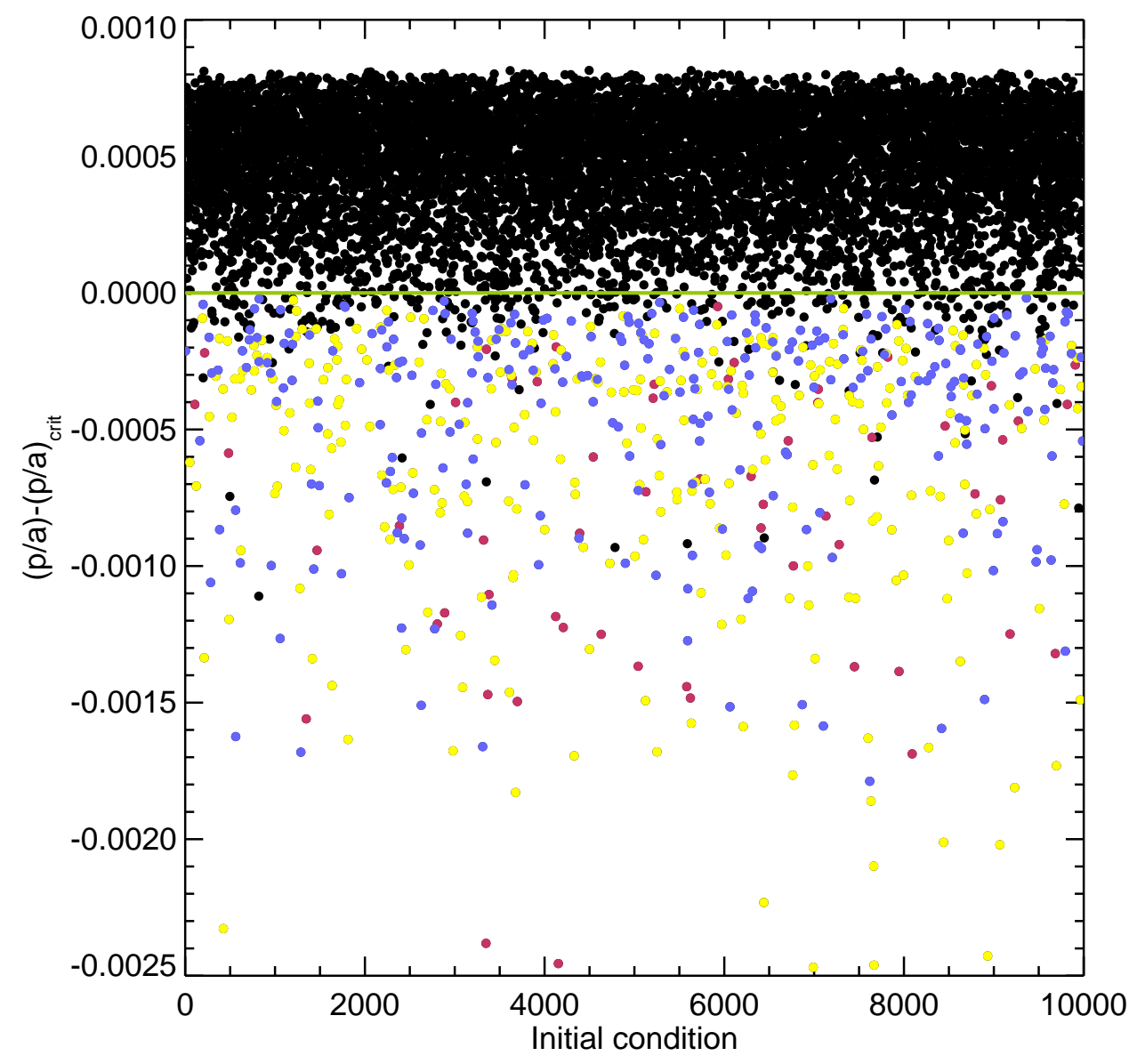

Figure S19: Hill and numerical stability diagram for the $10^{4}$ sets of initial conditions (ICs) drawn from the posterior distribution of the photo-dynamical model. The vertical axis is the dimensionless analytic Hill-stability criterion due to Marchal \& Bozis (1982) (12): positive values are Hill stable; horizontal axis is the index for the ICs we tested. The black dots indicate ICs that were stable after numerical integration for $2^{10}$ steps of $0.125 \mathrm{~d}$ per step $(6.84 \mathrm{Myr})$. The colored dots indicate the ICs that became unstable via close approach: blue became unstable after $>3 \times 10^{8}$ time steps, yellow went unstable between $3 \times 10^{7}-3 \times 10^{8}$ time steps, and red went unstable in $<3 \times 10^{7}$ time steps. 


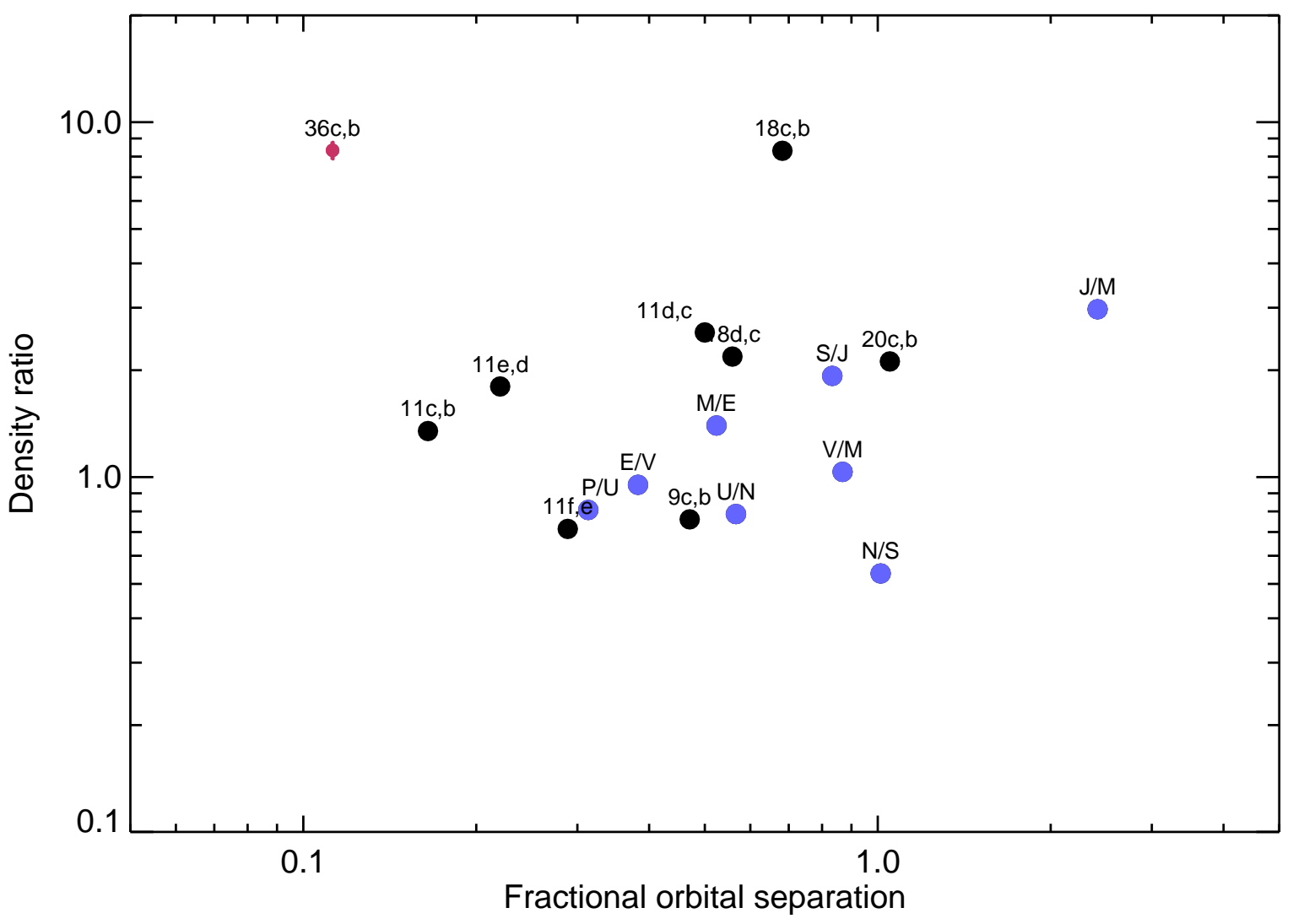

Figure S20: Distribution of fractional separation difference $\left(a_{2} / a_{1}-1\right)$ and density ratios $\left(\rho_{2} / \rho_{1}\right)$ for adjacent planets in our Solar System (blue) and in Kepler systems (black). Error bars are omitted since they are smaller than the size of the point for the Solar System, and are difficult to estimate for the Kepler systems (with the exception of Kepler-36). The labels use the first letter of the solar system planets, and the Kepler planet number, (i.e. 11e,b plots the ratio of Kepler-11f to Kepler 11e). Kepler-36 is plotted as a red point. 


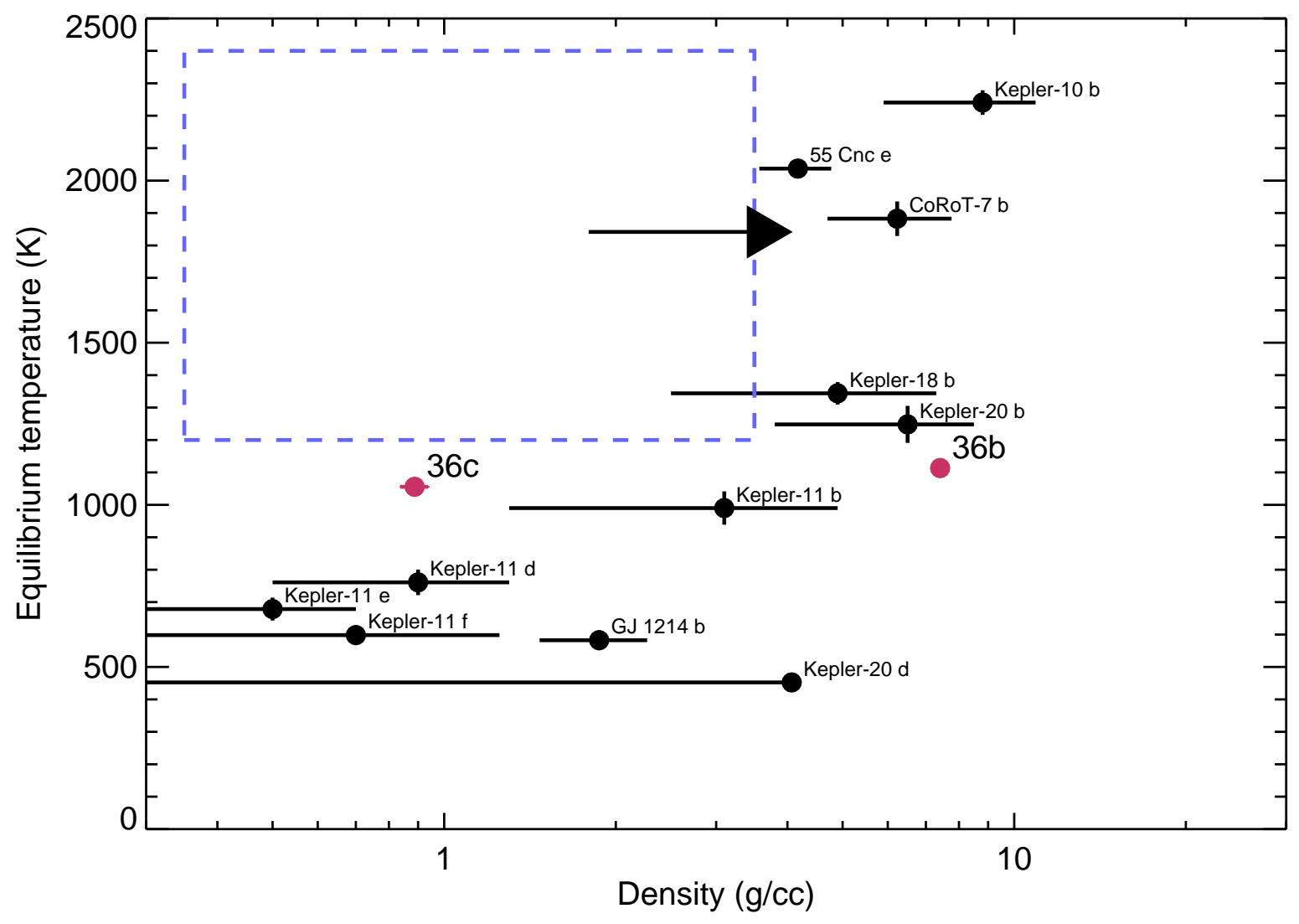

Figure S21: Distribution of densities and effective temperatures for super-Earths and miniNeptunes $\left(M_{p}<10 M_{\oplus}\right)$. We have indicated the lack of hot mini-Neptunes $(\rho<3.5 \mathrm{~g} / \mathrm{cc}$, $T<1200 K$ ) with a dashed blue box. 
We have attached the 10,001 initial conditions used in the stability analysis ( $\$$ (5) (as an ASCII file):

1223269s4.txt

We have attached a draw of 10,000 model parameters from our full posterior (as an ASCII text file - with FITS encoding for easier input):

1223269s5.txt

The authors welcome requests for additional information regarding the material presented in this paper.

\section{References and Notes}

1. Mayor, M. \& D. Queloz, A Jupiter-mass companion to a solar-type star. Nature 378, 355359 (1995).

2. Lin, D. N. C., P. Bodenheimer, \& D. C. Richardson, Orbital migration of the planetary companion of 51 Pegasi to its present location. Nature 380, 606-607 (1996).

3. Borucki, W. J., et al., Kepler Planet-Detection Mission: Introduction and First Results. Science 327, 977 (2010).

4. Koch, D. G., et al., Kepler Mission Design, Realized Photometric Performance, and Early Science. The Astrophysical Journal 713, L79-L86 (2010).

5. Caldwell, D. A., et al., Instrument Performance in Kepler's First Months. The Astrophysical Journal 713, L92-L96 (2010).

6. Jenkins, J. M., et al., Transiting planet search in the Kepler pipeline. Society of PhotoOptical Instrumentation Engineers (SPIE) Conference Series 7740, (2010).

7. Jenkins, J. M., et al., Overview of the Kepler Science Processing Pipeline. The Astrophysical Journal 713, L87-L91 (2010).

8. Kel'Manov, A. V. \& B. Jeon, A Posteriori Joint Detection and Discrimination of Pulses in a Quasiperiodic Pulse Train. IEEE Transactions on Signal Processing 52, 645-656 (2004).

9. Ford, E. B., et al., Transit Timing Observations from Kepler: II. Confirmation of Two Multiplanet Systems via a Non-parametric Correlation Analysis. ArXiv e-prints, arXiv:1201.5409-(2012). 
10. Steffen, J. H., et al., Transit timing observations from Kepler - III. Confirmation of four multiple planet systems by a Fourier-domain study of anticorrelated transit timing variations. Monthly Notices of the Royal Astronomical Society, 2482-(2012).

11. Fabrycky, D. C., et al., Transit Timing Observations from Kepler: IV. Confirmation of 4 Multiple Planet Systems by Simple Physical Models. ArXiv e-prints , arXiv:1201.5415(2012).

12. Marchal, C. \& G. Bozis, Hill Stability and Distance Curves for the General Three-Body Problem. Celestial Mechanics 26, 311-333 (1982).

13. Gladman, B., Dynamics of systems of two close planets. Icarus 106, 247-(1993).

14. Barnes, R. \& R. Greenberg, Stability Limits in Extrasolar Planetary Systems. The Astrophysical Journal 647, L163-L166 (2006).

15. Chaplin, W. J., et al., Ensemble Asteroseismology of Solar-Type Stars with the NASA Kepler Mission. Science 332, 213-(2011).

16. Supplementary Online Material.

17. Carter, J. A., et al., KOI-126: A Triply Eclipsing Hierarchical Triple with Two Low-Mass Stars. Science 331, 562-(2011).

18. Mandel, K. \& E. Agol, Analytic Light Curves for Planetary Transit Searches. The Astrophysical Journal 580, L171-L175 (2002).

19. ter Braak, C.J.F., Vrugt, J.A., 2008, A Markov Chain Monte Carlo version of the genetic algorithm Differential Evolution: easy Bayesian computing for real parameter spaces. Statistics and Computing 16, 239-249 (2006)

20. Marcus, R. A., S. T. Stewart, D. Sasselov, \& L. Hernquist, Collisional Stripping and Disruption of Super-Earths. The Astrophysical Journal 700, L118-L122 (2009).

21. Miralda-Escudé, J., Orbital Perturbations of Transiting Planets: A Possible Method to Measure Stellar Quadrupoles and to Detect Earth-Mass Planets. The Astrophysical Journal 564, 1019-1023 (2002).

22. Lissauer, J. J., et al., A closely packed system of low-mass, low-density planets transiting Kepler-11. Nature 470, 53-58 (2011). 
23. Cochran, W. D., et al., Kepler-18b, c, and d: A System of Three Planets Confirmed by Transit Timing Variations, Light Curve Validation, Warm-Spitzer Photometry, and Radial Velocity Measurements. The Astrophysical Journal Supplement Series 197, 7-(2011).

24. Gautier, T. N., et al., Kepler-20: A Sun-like star with three sub-Neptune exoplanets and two Earth-size candidates. The Astrophysical Journal 749, 15-(2012)

25. Charbonneau, D., et al., A super-Earth transiting a nearby low-mass star. Nature 462, 891-894 (2009).

26. Hatzes, A. P., et al., The Mass of CoRoT-7b. The Astrophysical Journal 743, 75-(2011).

27. Batalha, N. M., et al., Kepler's First Rocky Planet: Kepler-10b. The Astrophysical Journal 729, 27-(2011).

28. Winn, J. N., et al., A Super-Earth Transiting a Naked-eye Star. The Astrophysical Journal 737, L18-(2011).

29. Fortney, J. J., M. S. Marley, \& J. W. Barnes, Planetary Radii across Five Orders of Magnitude in Mass and Stellar Insolation: Application to Transits. The Astrophysical Journal 659, 1661-1672 (2007).

30. Buchhave, L., et al., Nature, DOI:10.1038/nature11121 (2012).

31. Kurucz, R. L. Model Atmospheres for Population Synthesis. IAU Symposium 149, 225 (1992).

32. Brown, T. M., R. L. Gilliland, R. W. Noyes, L. W. Ramsey, Detection of possible p-mode oscillations on Procyon. The Astrophysical Journal 368, 599-609 (1991).

33. Kjeldsen, H. T. R. Bedding, Amplitudes of stellar oscillations: the implications for asteroseismology.. Astronomy and Astrophysics 293, 87-106 (1995).

34. Christensen-Dalsgaard, J., On the Asteroseismic HR Diagram. GONG 1992. Seismic Investigation of the Sun and Stars 42, 347-350(1993).

35. Christensen-Dalsgaard, J., et al., Asteroseismic Investigation of Known Planet Hosts in the Kepler Field. The Astrophysical Journal 713, L164-L168 (2010).

36. Hekker, S., et al., The Octave (Birmingham-Sheffield Hallam) automated pipeline for extracting oscillation parameters of solar-like main-sequence stars. Monthly Notices of the Royal Astronomical Society 402, 2049-2059 (2010). 
37. Huber, D., et al., Automated extraction of oscillation parameters for Kepler observations of solar-type stars. Communications in Asteroseismology 160, 74-91(2009).

38. Verner, G. A., et al., Global asteroseismic properties of solar-like oscillations observed by Kepler: a comparison of complementary analysis methods. Monthly Notices of the Royal Astronomical Society 415, 3539-3551 (2011).

39. Fletcher, S. T., W. J. Chaplin, Y. Elsworth, R. New, Efficient Pseudo-Global Fitting for Helioseismic Data. The Astrophysical Journal 694, 144-150 (2009).

40. Handberg, R. T. L. Campante, Bayesian peak-bagging of solar-like oscillators using MCMC: a comprehensive guide. Astronomy and Astrophysics 527, A56 (2011).

41. Stello, D., et al., Radius Determination of Solar-type Stars Using Asteroseismology: What to Expect from the Kepler Mission. The Astrophysical Journal 700, 1589-1602 (2009).

42. Basu, S., W. J. Chaplin, Y. Elsworth, Determination of Stellar Radii from Asteroseismic Data. The Astrophysical Journal 710, 1596-1609 (2010).

43. Quirion, P.-O., J. Christensen-Dalsgaard, T. Arentoft, Automatic Determination of Stellar Parameters Via Asteroseismology of Stochastically Oscillating Stars: Comparison with Direct Measurements. The Astrophysical Journal 725, 2176-2189 (2010).

44. Gai, N., S. Basu, W. J. Chaplin, Y. Elsworth, An In-depth Study of Grid-based Asteroseismic Analysis. The Astrophysical Journal 730, 63 (2011).

45. Metcalfe, T. S. P. Charbonneau, Stellar structure modeling using a parallel genetic algorithm for objective global optimization. Journal of Computational Physics 185, 176-193 (2003).

46. Metcalfe, T. S., O. L. Creevey, J. Christensen-Dalsgaard, A Stellar Model-fitting Pipeline for Asteroseismic Data from the Kepler Mission. The Astrophysical Journal 699, 373-382 (2009).

47. Woitaszek, M., T. Metcalfe, I. Shorrock, AMP: a science-driven web-based application for the TeraGrid. Proceedings of the 5th Grid Computing Environments Workshop, p. 1-7 (2009).

48. Christensen-Dalsgaard, J., ASTEC - the Aarhus STellar Evolution Code. Astrophysics and Space Science 316, 13-24 (2008). 
49. Christensen-Dalsgaard, J., ADIPLS - the Aarhus adiabatic oscillation package. Astrophysics and Space Science 316, 113-120 (2008).

50. Alexander, D. R. J. W. Ferguson, Low-temperature Rosseland opacities. The Astrophysical Journal 437, 879-891 (1994).

51. Bahcall, J. N. M. H. Pinsonneault, Standard solar models, with and without helium diffusion, and the solar neutrino problem. Reviews of Modern Physics 64, 885-926 (1992).

52. Michaud, G. C. R. Proffitt, Particle transport processes. IAU Colloq. 137: Inside the Stars 40, 246-259 (1993).

53. Böhm-Vitense, E., Über die Wasserstoffkonvektionszone in Sternen verschiedener Effektivtemperaturen und Leuchtkräfte. Mit 5 Textabbildungen. Zeitschrift fur Astrophysik 46, 108-(1958).

54. Kjeldsen, H., T. R. Bedding, J. Christensen-Dalsgaard, Correcting Stellar Oscillation Frequencies for Near-Surface Effects. The Astrophysical Journal 683, L175-L178 (2008).

55. Creevey, O. L., et al., The Complementary Roles of Interferometry and Asteroseismology in Determining the Mass of Solar-Type Stars. The Astrophysical Journal 659, 616-625 (2007).

56. Gilliland, R. L., et al., Asteroseismology of the Transiting Exoplanet Host HD 17156 with Hubble Space Telescope Fine Guidance Sensor. The Astrophysical Journal 726, 2-(2011).

57. Batalha, N. M., et al., Kepler's First Rocky Planet: Kepler-10b. The Astrophysical Journal 729, 27-(2011).

58. Rogers, F. J., F. J. Swenson, C. A. Iglesias, OPAL Equation-of-State Tables for Astrophysical Applications. The Astrophysical Journal 456, 902-908 (1996).

59. Iglesias, C. A. F. J. Rogers, Updated Opal Opacities. The Astrophysical Journal 464, 943-953 (1996).

60. Ferguson, J. W., et al., Low-Temperature Opacities. The Astrophysical Journal 623, 585596 (2005).

61. Angulo, C., et al., A compilation of charged-particle induced thermonuclear reaction rates. Nuclear Physics A 656, 3-183 (1999).

62. Scuflaire, R., et al., The Liége Oscillation code. Astrophysics and Space Science 316, 149-154 (2008). 
63. Scuflaire, R., et al., CLÉS, Code Liégeois d'Évolution Stellaire. Astrophysics and Space Science 316, 83-91 (2008).

64. Grevesse, N. A. Noels, Atomic data and the spectrum of the solar photosphere.. Physica Scripta Volume T 47, 133-138 (1993).

65. Rogers, F. J. A. Nayfonov, Updated and Expanded OPAL Equation-of-State Tables: Implications for Helioseismology. The Astrophysical Journal 576, 1064-1074 (2002).

66. Demarque, P., D. B. Guenther, L. H. Li, A. Mazumdar, C. W. Straka, YREC: the Yale rotating stellar evolution code. Non-rotating version, seismology applications. Astrophysics and Space Science 316, 31-41 (2008).

67. Adelberger, E. G., et al., Solar fusion cross sections. Reviews of Modern Physics 70, 1265-1291 (1998).

68. Formicola, A., et al., Astrophysical S-factor of ${ }^{14} \mathrm{~N}(\mathrm{p}, \gamma){ }^{15} \mathrm{O}$. Physics Letters B 591, 61-68 (2004).

69. Thoul, A. A., J. N. Bahcall, A. Loeb, Element diffusion in the solar interior. The Astrophysical Journal 421, 828-842 (1994).

70. Basu, S., W. J. Chaplin, Y. Elsworth, R. New, A. M. Serenelli, Fresh Insights on the Structure of the Solar Core. The Astrophysical Journal 699, 1403-1417 (2009).

71. Deheuvels, S., et al., Seismic and spectroscopic characterization of the solar-like pulsating CoRoT target HD 49385. Astronomy and Astrophysics 515, A87-(2010).

72. Metcalfe, T. S., et al., A Precise Asteroseismic Age and Radius for the Evolved Sun-like Star KIC 11026764. The Astrophysical Journal 723, 1583-1598 (2010).

73. Tassoul, M., Asymptotic approximations for stellar nonradial pulsations. The Astrophysical Journal Supplement Series 43, 469-490 (1980).

74. Gough, D. O., EBK Quantization of Stellar Waves. Hydrodynamic and Magnetodynamic Problems in the Sun and Stars , 117-143(1986).

75. de Medeiros, J. R., J. D. Do Nascimento Jr., \& M. Mayor, On the link between rotation and lithium depletion in subgiant stars.. Astronomy and Astrophysics 317, 701-706 (1997).

76. Agol, E., J. Steffen, R. Sari, \& W. Clarkson, On detecting terrestrial planets with timing of giant planet transits. Monthly Notices of the Royal Astronomical Society 359, 567-579 (2005). 
77. Doyle, L. R., et al., Kepler-16: A Transiting Circumbinary Planet. Science 333, 1602(2011).

78. Welsh, W. F., et al., Transiting circumbinary planets Kepler-34 b and Kepler-35 b. Nature 481, 475-479 (2012).

79. Soderhjelm, S., Third-order and tidal effects in the stellar three-body problem. Astronomy and Astrophysics 141, 232-240 (1984).

80. Mardling, R. A. \& D. N. C. Lin, Calculating the Tidal, Spin, and Dynamical Evolution of Extrasolar Planetary Systems. The Astrophysical Journal 573, 829-844 (2002).

81. Press, W. H., S. A. Teukolsky, W. T. Vetterling, \& B. P. Flannery, Numerical recipes in $\mathrm{C}++$ : the art of scientific computing. (2002).

82. Ragozzine, D. \& M. J. Holman, The Value of Systems with Multiple Transiting Planets. ArXiv e-prints arXiv:1006.3727-(2010).

83. Rogers, L. A. \& S. Seager, A Framework for Quantifying the Degeneracies of Exoplanet Interior Compositions. The Astrophysical Journal 712, 974-991 (2010).

84. Rogers, L. A. \& S. Seager, Three Possible Origins for the Gas Layer on GJ 1214b. The Astrophysical Journal 716, 1208-1216 (2010).

85. Rogers, L. A., P. Bodenheimer, J. J. Lissauer, \& S. Seager, Formation and Structure of Low-density exo-Neptunes. The Astrophysical Journal 738, 59-(2011).

86. Marcus, R. A., D. Sasselov, L. Hernquist, \& S. T. Stewart, Minimum Radii of SuperEarths: Constraints from Giant Impacts. The Astrophysical Journal 712, L73-L76 (2010).

87. Lopez, E. D., J. J. Fortney, \& N. K. Miller, How Thermal Evolution and Mass Loss Sculpt Populations of Super-Earths and Sub-Neptunes: Application to the Kepler-11 System and Beyond. eprint arXiv:1205.0010, arXiv:1205.0010-(2012).

88. Miller, N. \& J. J. Fortney, The Heavy-element Masses of Extrasolar Giant Planets, Revealed. The Astrophysical Journal 736, L29-(2011).

89. Nettelmann, N., J. J. Fortney, U. Kramm, \& R. Redmer, Thermal Evolution and Structure Models of the Transiting Super-Earth GJ 1214b. The Astrophysical Journal 733, 2-(2011).

90. Thompson, S. L., 1990, "ANEOS—Analytic Equations of State for Shock Physics Codes," Sandia Natl. Lab. Doc. SAND89-2951 
91. Lyon, S. P. \& Johnson, J. D., 1992, LANL Rep. LA-UR-92-3407 (Los Alamos: LANL)

92. Saumon, D., G. Chabrier, \& H. M. van Horn, An Equation of State for Low-Mass Stars and Giant Planets. The Astrophysical Journal Supplement Series 99, 713-741(1995).

93. Nettelmann, N., et al., Ab Initio Equation of State Data for Hydrogen, Helium, and Water and the Internal Structure of Jupiter. The Astrophysical Journal 683, 1217-1228 (2008).

94. Lammer, H., et al., Atmospheric Loss of Exoplanets Resulting from Stellar X-Ray and Extreme-Ultraviolet Heating. The Astrophysical Journal 598, L121-L124 (2003).

95. Erkaev, N. V., et al., Roche lobe effects on the atmospheric loss from "Hot Jupiters". Astronomy and Astrophysics 472, 329-334 (2007).

96. Ribas, I., E. F. Guinan, M. Güdel, \& M. Audard, Evolution of the Solar Activity over Time and Effects on Planetary Atmospheres. I. High-Energy Irradiances. The Astrophysical Journal 622, 680-694 (2005).

97. Wisdom, J. \& M. Holman, Symplectic maps for the n-body problem. The Astronomical Journal 102, 1528-1538 (1991).

98. Wisdom, J., Symplectic Correctors for Canonical Heliocentric n-Body Maps. The Astronomical Journal 131, 2294-2298 (2006).

99. Hamilton, D. P. \& J. A. Burns, Orbital stability zones about asteroids. Icarus 92, 118-131 (1991). 